\title{
1,2-Hydroboration of Pyridines by Organomagnesium
}

\author{
Xinli Liu, ${ }^{1}$ Bingwen Li, ${ }^{2}$ Xiufang Hua,,${ }^{1,3}$ and Dongmei Cui*,1,3
}

${ }^{1}$ State Key Laboratory of Polymer Physics and Chemistry, Changchun Institute of Applied Chemistry, Chinese Academy of Sciences, Changchun 130022, China

${ }^{2}$ Laboratory of Theoretical and Computational Chemistry, Institute of Theoretical Chemistry, Jilin University, Changchun 130023, China

${ }^{3}$ University of Chinese Academy of Sciences, Changchun Branch, Changchun 130022, China

\section{Table of Contents}

1. EXPERIMENTS 2

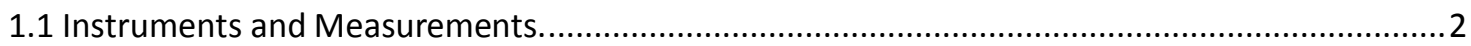

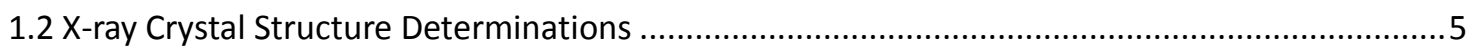

1.3 Characterization Data for Hydroboration Products............................................................. 10

1.4 Procedure for Typical NMR-Scale Catalytic Reaction ......................................................... 11

1.4.1 Spectroscopic Characterization of ligands, complexes and 2a-2w.....................................12

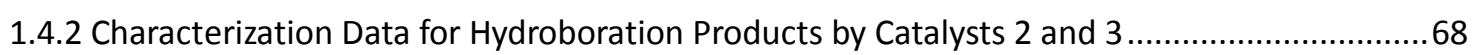

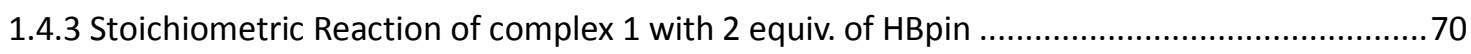

1.4.4 Stacking ${ }^{1} \mathrm{H}-\mathrm{NMR}$ Spectra for monitoring the initial reaction process ..................................72

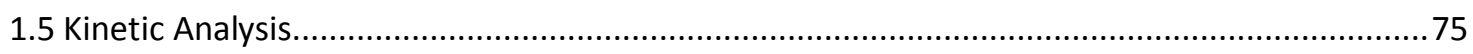

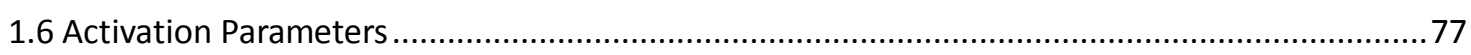

1.7 Determination of the Kinetic Isotope Effect...................................................................... 78

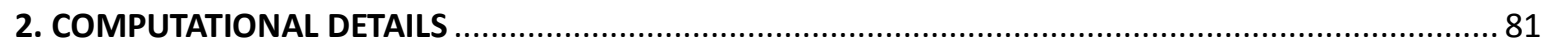

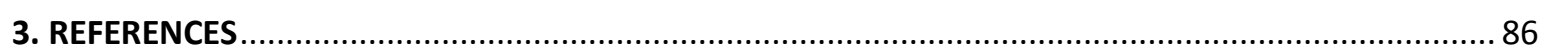




\section{EXPERIMENTS}

\subsection{Instruments and Measurements.}

NMR spectra of complexes were performed in $\mathrm{C}_{6} \mathrm{D}_{6}$ at $25{ }^{\circ} \mathrm{C}$ on a Bruker Avance 400 spectrometer or Bruker Avance 500 (direct cryoprobe, $500 \mathrm{MHz}$ ) instruments. Crystals for X-ray analysis were obtained as described in the Experimental section. The crystals were manipulated in a glovebox. Data collections were performed at $-86.5^{\circ} \mathrm{C}$ on a Bruker SMART APEX diffractometer with a CCD area detector, using graphite-monochromated Mo Ka radiation $(\lambda=$ 0.71073A). The determination of crystal class and unit cell parameters was carried out by the SMART program package. The raw frame data were processed using SAINT and SADABS to yield the reflection data file. The structures were solved by using the SHELXTL program. Refinement was performed on $F^{2}$ anisotropically for all non-hydrogen atoms by the full-matrix least-squares method. The hydrogen atoms were placed at the calculation without further refinement of the parameters.

The synthetic route from HL1 to complex 1 was reported by our groups earlier in literature. ${ }^{[1]}$ Complexes 2-3 were synthesized from the corresponding ligands with similar processes.

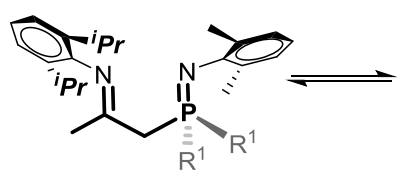

HL1. $R^{1}=C y, \quad H L 2 . R^{1}=P h$

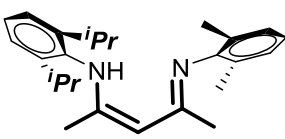

HL3

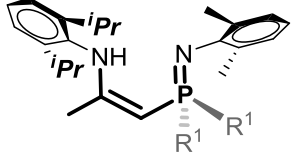

$\underset{\text { 1,MgBu}}{\stackrel{\mathrm{Mg}_{2}}{2}, \mathrm{PhSiH}_{3}}$

hexane/toluene

Complexes 1-2
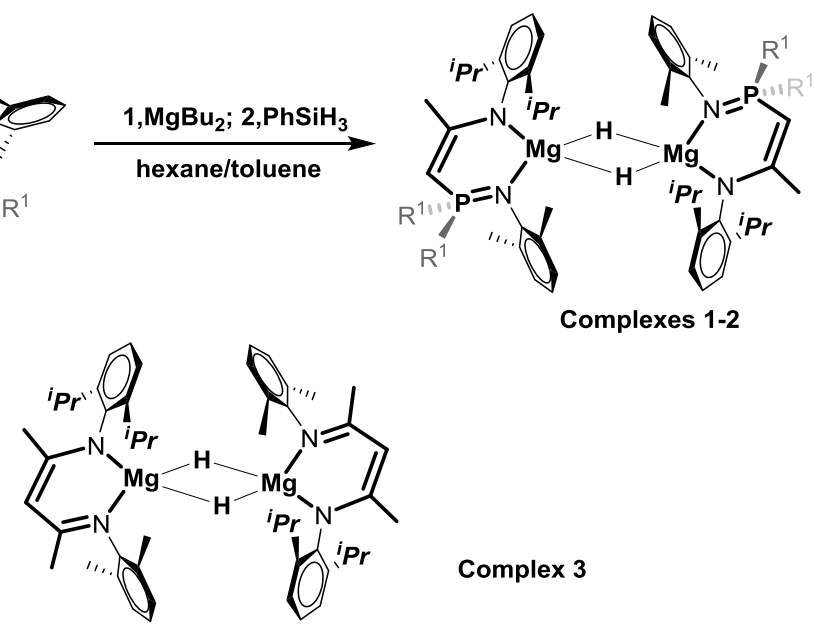

Complex 3

\section{Synthesis of HL1-HL3}

HL1-HL2: $\mathrm{Ar}_{-} \mathrm{N}_{3}$ was added dropwise to THF $(25 \mathrm{~mL})$ solution of $12.2 \mathrm{mmol} 2,6-{ }^{i} \operatorname{Pr}_{2}-$ $\mathrm{C}_{6} \mathrm{H}_{3} N=\mathrm{C}(\mathrm{Me}) \mathrm{CH}_{2} \mathrm{P}(\mathrm{R})_{2}$ in $25 \mathrm{~mL}$ THF solution. $14.6 \mathrm{mmol}$. $\mathrm{N}_{2}$ evolution commenced immediately and the mixture was stirred at $25^{\circ} \mathrm{C}$ for $24 \mathrm{~h}$. Removal of THF under vacuum left 
brown oily solid. The solid was then washed with $30 \mathrm{~mL}$ hexane and filtered to afford HL1 or HL2 as white powder.

HL3 was synthesized following a published procedure ${ }^{[2]}$.

$\left(2,6^{i}{ }^{i} r_{2}-C_{6} H_{3}\right) \mathrm{NC}(\mathrm{Me})-\mathrm{CH}_{2} P\left(\mathrm{Cy}_{2}\right)=\mathrm{N}\left(2,6-\mathrm{Me}_{2}-\mathrm{C}_{6} \mathrm{H}_{3}\right)(\mathrm{HL1}):$

White solid, weight: $5.25 \mathrm{~g}$, yield: $81 \%$.

${ }^{1} \mathrm{H}$ NMR (400 MHz, $\mathrm{CDCl}_{3}, 25{ }^{\circ} \mathrm{C}$ major isomer): $\delta=7.23-7.11\left(\mathrm{~m}, 3 \mathrm{H}, \mathrm{C}_{6} H_{3}{ }_{3} \operatorname{Pr}_{2}\right), 7.03\left(\mathrm{~d}, J_{\mathrm{H}-\mathrm{H}}\right.$ $=8.0 \mathrm{~Hz}, 1 \mathrm{H}, \mathrm{Ar}), 6.88(\mathrm{~m}, 1 \mathrm{H}, \mathrm{Ar}), 3.02\left(\mathrm{~d},{ }^{2} J_{\mathrm{P}-\mathrm{H}}=12.0 \mathrm{~Hz}, 2 \mathrm{H}, \mathrm{PCH} 2\right), 2.87\left(\mathrm{sept}, J_{\mathrm{H}-\mathrm{H}}=4.0\right.$ $\left.\mathrm{Hz}, 2 \mathrm{H}, \mathrm{CH}\left(\mathrm{CH}_{3}\right)_{2}\right), 2.48$ (s, 6H, $\mathrm{ArCH}_{3}$ ), 1.61-1.53 (br, 12H, Cy), 1.53 (s, 3H, MeCCH $\mathrm{CH}_{2} \mathrm{P}$, $1.14-1.07\left(\mathrm{dd}, J_{\mathrm{H}-\mathrm{H}}=8.0 \mathrm{~Hz}, 12 \mathrm{H}, \mathrm{CH}\left(\mathrm{CH}_{3}\right)_{2}\right) \mathrm{ppm}$.

Anal. Calc. for $\mathrm{C}_{35} \mathrm{H}_{53} \mathrm{~N}_{2} \mathrm{P}: \mathrm{C}, 78.90 ; \mathrm{H}, 10.03 ; \mathrm{N}, 5.26$; Found C, 78.80; H, 9.91; N 5.21.

$\left(2,6-{ }^{i} \mathrm{Pr}_{2}-\mathrm{C}_{6} \mathrm{H}_{3}\right) \mathrm{NC}(\mathrm{Me})-\mathrm{CH}_{2} P\left(\mathrm{Ph}_{2}\right)=\mathrm{N}\left(2,6-\mathrm{Me}_{2}-\mathrm{C}_{6} \mathrm{H}_{3}\right)(\mathrm{HL} 2)$ :

White solid, weight: $4.68 \mathrm{~g}$, yield: $78 \%$.

${ }^{1} \mathrm{H}$ NMR (400 MHz, $\mathrm{CDCl}_{3}, 25{ }^{\circ} \mathrm{C}$, major isomer) : $\delta 7.78-7.68\left(\mathrm{~m}, 10 \mathrm{H}, \mathrm{PPh}_{2}\right), 7.15-6.95(\mathrm{~m}$, $6 \mathrm{H}, m, p$-Ar), $3.66\left(\mathrm{~d},{ }^{2} J_{\mathrm{P}-\mathrm{H}}=12.0 \mathrm{~Hz}, 2 \mathrm{H}, \mathrm{PCH} 2\right), 2.67\left(\mathrm{sept}, J_{\mathrm{H}-\mathrm{H}}=4.0 \mathrm{~Hz}, 2 \mathrm{H}, \mathrm{CH}\left(\mathrm{CH}_{3}\right)_{2}\right), 2.40$ (s, 6H, $\left.\mathrm{ArCH}_{3}\right), 1.51\left(\mathrm{~s}, 3 \mathrm{H}, \mathrm{MeCCH}_{2} \mathrm{P}\right), 1.09-1.01\left(\mathrm{dd}, J_{\mathrm{H}-\mathrm{H}}=8.0 \mathrm{~Hz}, 12 \mathrm{H}, \mathrm{CH}\left(\mathrm{CH}_{3}\right)_{2}\right) \mathrm{ppm}$. ${ }^{31} \mathrm{P} \mathrm{NMR}\left(162 \mathrm{MHz}, \mathrm{CDCl}_{3}, 25^{\circ} \mathrm{C}\right): \delta 1.97$ (67\%, major isomer), -3.30 (33\%, minor isomer) ppm. ${ }^{13} \mathrm{C} \mathrm{NMR}\left(100 \mathrm{MHz}, \mathrm{CDCl}_{3}, 25{ }^{\circ} \mathrm{C}\right.$, major isomer): $\delta=164.6\left(\mathrm{~d},{ }^{2} \mathrm{~J}_{\mathrm{P}-\mathrm{C}}=6 \mathrm{~Hz}, \mathrm{CN}\right), 161.4$ (imine, ipso-Ar), 136.1 (d, $\left.{ }^{3} J_{\mathrm{P}-\mathrm{C}}=2 \mathrm{~Hz}, \mathrm{PAr}_{2}\right), 135.7(\mathrm{Ar}), 134.9(\mathrm{Ar}), 131.0\left(\mathrm{~d},{ }^{3} J_{\mathrm{P}-\mathrm{C}}=8 \mathrm{~Hz}\right.$, ipso-Ar), $130.7(\mathrm{Ar}), 130.1(\mathrm{Ar}), 128.3(\mathrm{Ar}), 123.8(\mathrm{Ar}), 123.3(\mathrm{Ar}), 123.0(\mathrm{Ar}), 118.6(\mathrm{Ar}), 45.6\left(\mathrm{~d}, J_{\mathrm{P}-\mathrm{C}}=\right.$ $\left.50 \mathrm{~Hz}, \mathrm{CH}_{2}\right), 28.3(\mathrm{CH}), 27.7(\mathrm{Me}), 23.6$. (Me) ppm.

Anal. Calc. for $\mathrm{C}_{35} \mathrm{H}_{41} \mathrm{~N}_{2} \mathrm{P}: \mathrm{C}, 80.73 ; \mathrm{H}, 7.94 ; \mathrm{N}$, 5.38. Found C, 80.68, H, 7.75; N, 5.33.

$\left(2,6-{ }^{i} \mathrm{Pr}_{2}-\mathrm{C}_{6} \mathrm{H}_{3}\right) \mathrm{NHC}(\mathrm{Me})=\mathrm{CHC}(\mathrm{Me})=\mathrm{N}\left(2,6-\mathrm{Me}_{2}-\mathrm{C}_{6} \mathrm{H}_{3}\right)(\mathrm{HL} 3):$

White needle-like crystal, weight 3.2 g, yield $71 \%$.

${ }^{1} \mathrm{H}$ NMR $\left(400 \mathrm{MHz}, \mathrm{CDCl}_{3}, 25^{\circ} \mathrm{C}\right): \delta=12.54(1 \mathrm{H}, \mathrm{NH}), 7.15-7.10\left(\mathrm{~m}, 3 \mathrm{H}, \mathrm{C}_{6} \mathrm{H}_{3}{ }^{i} \operatorname{Pr}_{2}\right), 6.93-6.99$ (m, 3H, Ar), 7.03 (m, 2H, Ar), $6.97(\mathrm{~m}, 1 \mathrm{H}, \mathrm{Ar}), 4.85$ (s, 1H, CH), 3.23 (sept, $J_{\mathrm{H}-\mathrm{H}}=4.0 \mathrm{~Hz}, 2 \mathrm{H}$, $\left.\mathrm{CH}\left(\mathrm{CH}_{3}\right)_{2}\right), 2.18\left(\mathrm{~s}, 6 \mathrm{H}, \mathrm{ArCH}_{3}\right), 1.66(\mathrm{~s}, 3 \mathrm{H}, \mathrm{MeC}), 1.58(\mathrm{~s}, 3 \mathrm{H}, \mathrm{MeC}), 1.21-1.10\left(\mathrm{dd}, J_{\mathrm{H}-\mathrm{H}}=4.0\right.$ $\left.\mathrm{Hz}, 12 \mathrm{H}, \mathrm{CH}\left(\mathrm{CH}_{3}\right)_{2}\right) \mathrm{ppm}$.

${ }^{13} \mathrm{C} \mathrm{NMR}\left(100 \mathrm{MHz}, \mathrm{CDCl}_{3}, 25{ }^{\circ} \mathrm{C}\right.$, major isomer): $\delta=161.6$ (ipso-Ar), 160.0 (ipso-Ar), 144.7 , 142.8 142.4, 140.0, 131.3, 125.7, 124.1, 123.2, 123.1 (Ar), $93.8(\mathrm{CH}), 28.4\left(\mathrm{CHMe}_{2}\right), 24.1(\mathrm{Me})$, $22.6(\mathrm{Me}), 20.1(\mathrm{Me}), 18.0(\mathrm{Me}) \mathrm{ppm}$. 
Anal. Calc. for $\mathrm{C}_{25} \mathrm{H}_{34} \mathrm{~N}_{2}$ : C, 82.82; H, 9.45; N, 7.73. Found: C, 82.79; H, 9.33; N, 7.68.

\section{Synthesis of Complexes 1-3}

Complexes 1-3 were prepared according to the our reported method with revised procedure. ${ }^{[1]}$ HL (1 mmol in $10 \mathrm{~mL}$ toluene) was added dropwise to $\mathrm{Mg}^{n} \mathrm{Bu}_{2}(1.1 \mathrm{~mL}, 1 \mathrm{M}, 1.1 \mathrm{mmol})$ within $10 \mathrm{~mL}$ hexane solution at room temperature. The mixture was stirred for $4 \mathrm{~h}$ at this temperature to afford a clear pale yellow solution. Then phenylsilane $(1 \mathrm{mmol})$ was added and stirred for another $24 \mathrm{~h}$ at same temperature. The obtaining white suspension was filtered and washed with hexane $(3 \times 5 \mathrm{~mL})$ to provide corresponding magnesium hydride complexes as white powder. The single crystals of products were obtained from hexane/toluene mixed solvents at $-35^{\circ} \mathrm{C}$. Complex 1 was reported by our group earlier ${ }^{[1]}$

\section{Complex 1:}

White solid, weight $0.34 \mathrm{~g}$, yield $62 \%$

${ }^{1} \mathrm{H}$ NMR (400 MHz, $\left.\mathrm{C}_{6} \mathrm{D}_{6}, 25{ }^{\circ} \mathrm{C}\right): \delta 7.21-7.16(\mathrm{~m}, 4 \mathrm{H}, \mathrm{Ar}), 7.10-7.08(\mathrm{~m}, 2 \mathrm{H}, \mathrm{Ar}), 6.95-6,87(\mathrm{~m}$, $6 \mathrm{H}, \mathrm{Ar}), 3.84\left(\mathrm{~s}, 2 \mathrm{H}, \mathrm{Mg} \mathrm{H}_{2} \mathrm{Mg}\right), 3.51\left(\mathrm{sept}, 4 \mathrm{H},{ }^{3} \mathrm{~J}_{\mathrm{H}-\mathrm{H}}=8.0 \mathrm{~Hz}, \mathrm{CH}\left(\mathrm{CH}_{3}\right)_{2}\right), 3.02\left(\mathrm{~d},{ }^{2} J_{\mathrm{P}-\mathrm{H}}=24.0\right.$ $\mathrm{Hz}, 2 \mathrm{H}, \mathrm{PCH}$ ), 2.29 (m, 4H, ipso-Cy), 2.19 (s, 6H, $\left.\mathrm{NCCH}_{3}\right), 1.55$ (s, 12H, $\left.\mathrm{C}_{6} \mathrm{H}_{3}\left(\mathrm{CH}_{3}\right)_{2}\right), 1.33$ (d, $\left.{ }^{3} J_{\mathrm{H}-\mathrm{H}}=8.0 \mathrm{~Hz}, 12 \mathrm{H}, \mathrm{CH}\left(\mathrm{CH}_{3}\right)_{2}\right), 1.06\left(\mathrm{~m}, 12 \mathrm{H}, \mathrm{CH}\left(\mathrm{CH}_{3}\right)_{2}\right), 2.12-0.97(\mathrm{~m}, 40 \mathrm{H}, \mathrm{PCy})_{2}$.

${ }^{31} \mathrm{P}$ NMR (162 MHz, $\left.\mathrm{C}_{6} \mathrm{D}_{6}, 25^{\circ} \mathrm{C}\right): \delta 47.76 \mathrm{ppm}(\mathrm{s})$.

Anal. Calcd for $\mathrm{C}_{70} \mathrm{H}_{106} \mathrm{Mg}_{2} \mathrm{~N}_{4} \mathrm{P}_{2}$ : C, 75.46; H, 9.59; N, 5.03. Found: C, 75.29; H, 9.52; N, 5.07.

\section{Complex 2:}

White solid, weight $0.37 \mathrm{~g}$, yield $67 \%$

${ }^{1} \mathrm{H}$ NMR $\left(400 \mathrm{MHz}, \mathrm{C}_{6} \mathrm{D}_{6}, 25^{\circ} \mathrm{C}\right): \delta 7.72-7.69$ (sept, $\left.J_{\mathrm{H}-\mathrm{H}}=4.0 \mathrm{~Hz}, 8 \mathrm{H}, \mathrm{Ar}\right), 7.07-7.01(\mathrm{~m}, 12 \mathrm{H}$, $\operatorname{Ar}), 6.94(\mathrm{~m}, 2 \mathrm{H}, \mathrm{Ar}), 6.86-6.80\left(\mathrm{dd}, J_{\mathrm{H}-\mathrm{H}}=4.0 \mathrm{~Hz}, 8 \mathrm{H}, \mathrm{Ar}\right), 6.75(\mathrm{~m}, 2 \mathrm{H}, \mathrm{Ar}), 4.00\left(\mathrm{~d},{ }^{2} J_{\mathrm{P}-\mathrm{H}}=\right.$ $20.0 \mathrm{~Hz}, 2 \mathrm{H}, \mathrm{PCH}), 3.80$ (s, $\left.2 \mathrm{H}, \mathrm{MgH} \mathrm{H}_{2} \mathrm{Mg}\right), 2.85$ (sept, $\left.J_{\mathrm{H}-\mathrm{H}}=4.0 \mathrm{~Hz}, 4 \mathrm{H}, \mathrm{CH}\left(\mathrm{CH}_{3}\right)_{2}\right), 2.32(\mathrm{~s}$, $\left.12 \mathrm{H}, \mathrm{ArCH}_{3}\right), 1.51(\mathrm{~s}, 6 \mathrm{H}, \mathrm{MeCN}), 1.07-0.98\left(\mathrm{dd}, J_{\mathrm{H}-\mathrm{H}}=8.0 \mathrm{~Hz}, 24 \mathrm{H}, \mathrm{CH}\left(\mathrm{CH}_{3}\right)_{2}\right) \mathrm{ppm}$. ${ }^{31} \mathrm{P}$ NMR (162 MHz, $\left.\mathrm{C}_{6} \mathrm{D}_{6}, 25^{\circ} \mathrm{C}\right): \delta 20.39 \mathrm{ppm}$.

${ }^{13} \mathrm{C}$ NMR $\left(100 \mathrm{MHz}, \mathrm{C}_{6} \mathrm{D}_{6}, 25{ }^{\circ} \mathrm{C}\right): \delta=173.4(\mathrm{CN}), 146.3$ (imine, ipso-Ar), $144.7\left(\mathrm{~d},{ }^{3} J_{\mathrm{P}-\mathrm{C}}=2.0\right.$ $\left.\mathrm{Hz}, \mathrm{PAr}_{2}\right), 143.5(\mathrm{Ar}), 136.0\left(\mathrm{~d},{ }^{3} J_{\mathrm{P}-\mathrm{C}}=8.0 \mathrm{~Hz}\right.$, ipso-Ar), 135.4, 134.7, 132.6, 132.5, 130.3, 127.7, 127.4, 127.3, 123.6, 123.1, $121.7(\mathrm{Ar}), 67.2-66.9\left(\mathrm{~d}, J_{\mathrm{P}-\mathrm{C}}=29 \mathrm{~Hz}, \mathrm{NCCH}_{3}\right), 59.0-57.9\left(\mathrm{~d}, J_{\mathrm{P}-\mathrm{C}}=\right.$ $110 \mathrm{~Hz}, \mathrm{CH}), 27.1\left(\mathrm{CH}_{3}\right), 25.3\left(\mathrm{CH}\left(\mathrm{CH}_{3}\right)_{2}\right), 19.8\left(\mathrm{CH}_{3}\right)$ ppm. 
Anal. Calc. for $\mathrm{C}_{70} \mathrm{H}_{82} \mathrm{Mg}_{2} \mathrm{~N}_{4} \mathrm{P}_{2}$ : C, 77.13; H, 7.58; N, 5.14. Found: C, 77.10; H, 7.46; N, 5.07.

\section{Complex 3:}

White solid, weight $0.27 \mathrm{~g}$, yield $71 \%$

${ }^{1} \mathrm{H}$ NMR (400 MHz, $\left.\mathrm{C}_{6} \mathrm{D}_{6}, 2{ }^{\circ} \mathrm{C}\right): \delta=7.15\left(\mathrm{~m}, 2 \mathrm{H}, \mathrm{C}_{6} H_{3}{ }^{i} \mathrm{Pr}_{2}\right), 7.08\left(\mathrm{t}, J_{\mathrm{H}-\mathrm{H}}=4.0 \mathrm{~Hz}, 2 \mathrm{H}, \mathrm{Ar}\right)$, $6.99\left(\mathrm{~d}, J_{\mathrm{H}-\mathrm{H}}=4.0 \mathrm{~Hz}, 4 \mathrm{H}, \mathrm{Ar}\right), 6.89(\mathrm{~m}, 4 \mathrm{H}, \mathrm{Ar}), 4.79(\mathrm{~s}, 2 \mathrm{H}, \mathrm{CH}), 3.81\left(\mathrm{~s}, 2 \mathrm{H}, \mathrm{MgH} \mathrm{H}_{2} \mathrm{Mg}\right), 3.11$ (sept, $\left.J_{\mathrm{H}-\mathrm{H}}=4.0 \mathrm{~Hz}, 4 \mathrm{H}, \mathrm{CH}\left(\mathrm{CH}_{3}\right)_{2}\right), 1.88\left(\mathrm{~s}, 12 \mathrm{H}, \mathrm{ArCH}_{3}\right), 1.52(\mathrm{~s}, 6 \mathrm{H}, \mathrm{MeC}), 1.37(\mathrm{~s}, 6 \mathrm{H}, \mathrm{MeC})$, $1.11-1.02\left(\mathrm{dd}, J_{\mathrm{H}-\mathrm{H}}=4.0 \mathrm{~Hz}, 24 \mathrm{H}, \mathrm{CH}\left(\mathrm{CH}_{3}\right)_{2}\right) \mathrm{ppm}$.

${ }^{13} \mathrm{C}$ NMR (100 MHz, $\mathrm{C}_{6} \mathrm{D}_{6}, 25{ }^{\circ} \mathrm{C}$ ): $\delta 168.9$ (ipso-Ar), 168.3 (ipso-Ar), 147.5, 144.0. 141.7, 131.4,

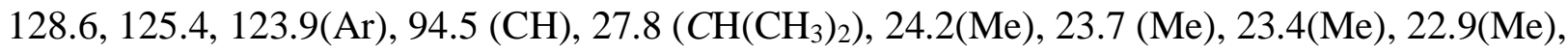
18.1(Me) ppm.

Anal. Calc. for $\mathrm{C}_{50} \mathrm{H}_{68} \mathrm{Mg}_{2} \mathrm{~N}_{4}$ : C, 77.62; H, 8.86; N, 7.24. Found: C, 77.59; H, 8.65; N, 7.17.

\section{General procedures for the hydroboration of $N$-heteroarenes with NMR-Scale}

In a glove box, complex $1(4.2 \mathrm{mg}, 3.77 \mu \mathrm{mol})$ and triphenylmethylsilane $(5.5 \mathrm{mg}, 20 \mu \mathrm{mol})$ were added to a NMR tube with $0.5 \mathrm{~mL}$ of $\mathrm{C}_{6} \mathrm{D}_{6}$ and then put into refrigerator at $-35^{\circ} \mathrm{C}$ before use. $\mathrm{N}$ heteroarenes $(0.189 \mathrm{mmol})$ and HBpin $(0.189 \mathrm{mmol}, 29 \mu \mathrm{L})$ were quickly transferred to the NMR tube through micro-injector and then shaken just before NMR measurement. The related concentrations of pyridines and products were determined relative to the intensity of the internal standard resonance.

\section{Preparative Scale Reaction Procedure}

In a glove box, complex 1 (4.4 mg, $4 \mu \mathrm{mol})$ and triphenylmethylsilane $(5.5 \mathrm{mg}, 20 \mu \mathrm{mol})$ were added to an $25 \mathrm{~mL}$ flask with a stir bar and dissolved in dry benzene (5mL). 2-acetyl-pyridine (4 mmol, $0.448 \mathrm{~mL})$ and HBpin $(4 \mathrm{mmol}, 0.61 \mathrm{~mL})$ were added. The flask was stirred at r.t. for 2 hours before the volatiles were removed in vacuo. The obtained solid was then dissolved in hexane and filtered through a short pad of celite. After evaporated the filtrate, $0.90 \mathrm{~g}$ (91\% yield) white solid of 2-(1-((4,4,5,5-tetramethyl-1,3,2-dioxaborolan-2-yl)oxy)ethyl)pyridine (2t) was obtained.

\subsection{X-ray Crystal Structure Determinations}

Crystals of 2 was obtained by recrystallization of crude products from toluene/hexane mixed 5 / 86 
solutions at $-35^{\circ} \mathrm{C}$. Crystal of $\mathbf{2 t}$ was directly acquired from the reaction solution a few days later. Data collections were performed at $-86.5{ }^{\circ} \mathrm{C}$ on a Bruker SMART APEX diffractometer with a CCD area detector, using graphite-monochromated Mo $\mathrm{K} \alpha$ radiation $(\lambda=0.71073 \AA)$. The determination of crystal class and unit cell parameters was carried out by the SMART program package. ${ }^{[3]}$ The raw frame data were processed using SAINT and SADABS to yield the reflection data file. ${ }^{[4]}$ The structures were solved by using the SHELXTL program. ${ }^{[5]}$ Refinement was performed on $F^{2}$ anisotropically for all non-hydrogen atoms by the full-matrix least-squares method. CCDC numbers of 1868409 (2) and 1890791 (2t). The same structure of $\mathbf{2 t}$ was individually reported by Y. Lebedev (Y. Lebedev, 2019. CSD Communication (Private Communication)) as CCDC number of 1888986. CCDC numbers of 1041464 (1) and 1041465 (4) are early reported by our group. ${ }^{[6]}$ These data are provided free of charge by The Cambridge Crystallographic Data Centre.

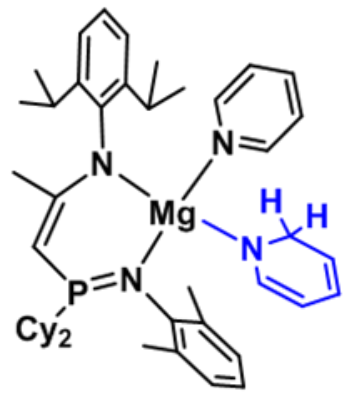

Complex 4

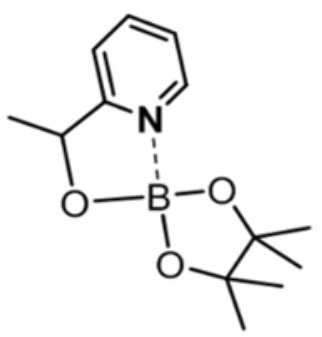

$2 t$ 


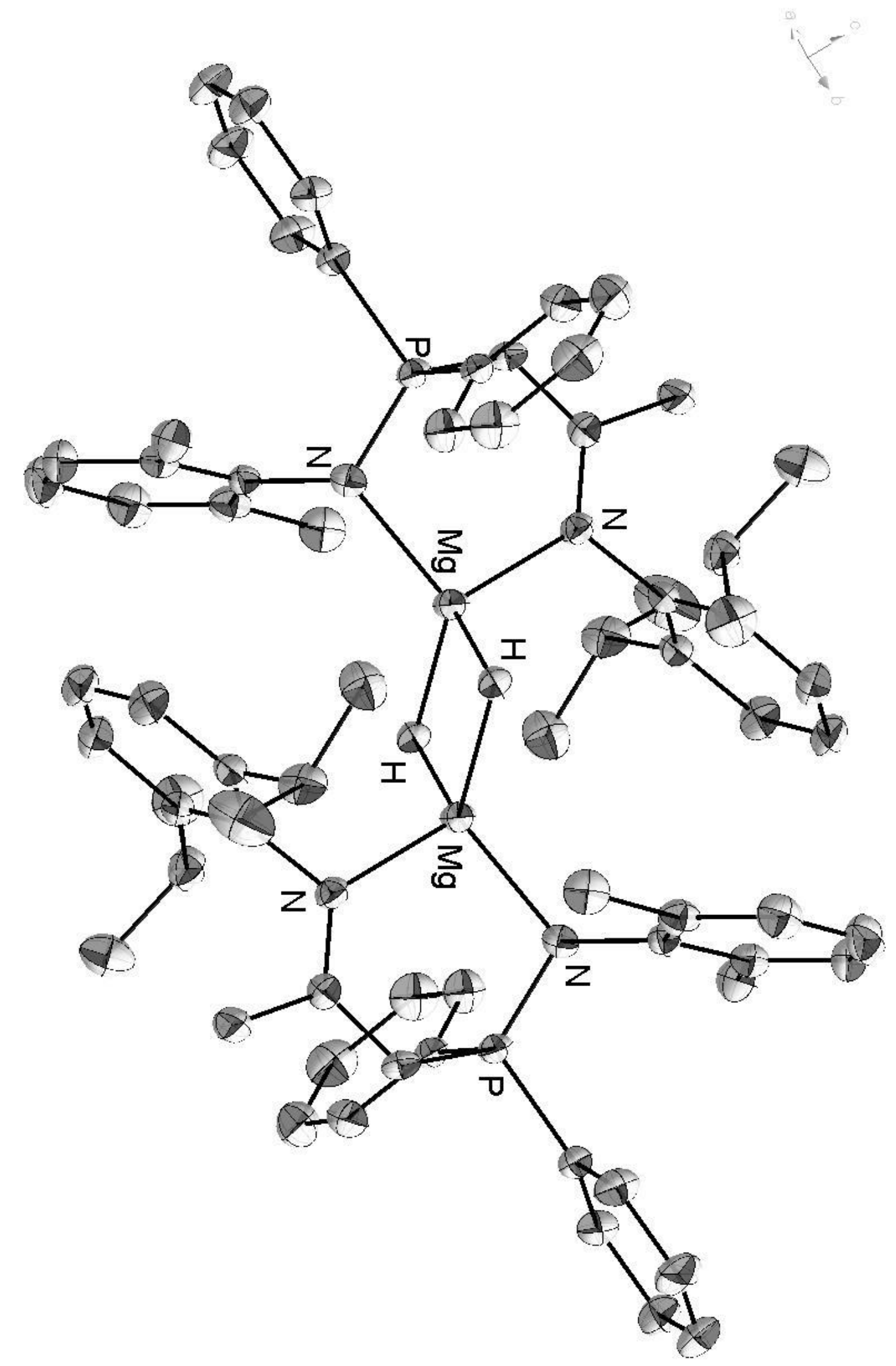

Figure S1. ORTEP plot of the molecular structure of complex 4. Thermal ellipsoids are drawn at the $35 \%$ probability level. $\mathrm{H}$ atoms are omitted for clarity, except for the $\mathrm{Mg}-\mathrm{H}$ bridges. 7 / 86 


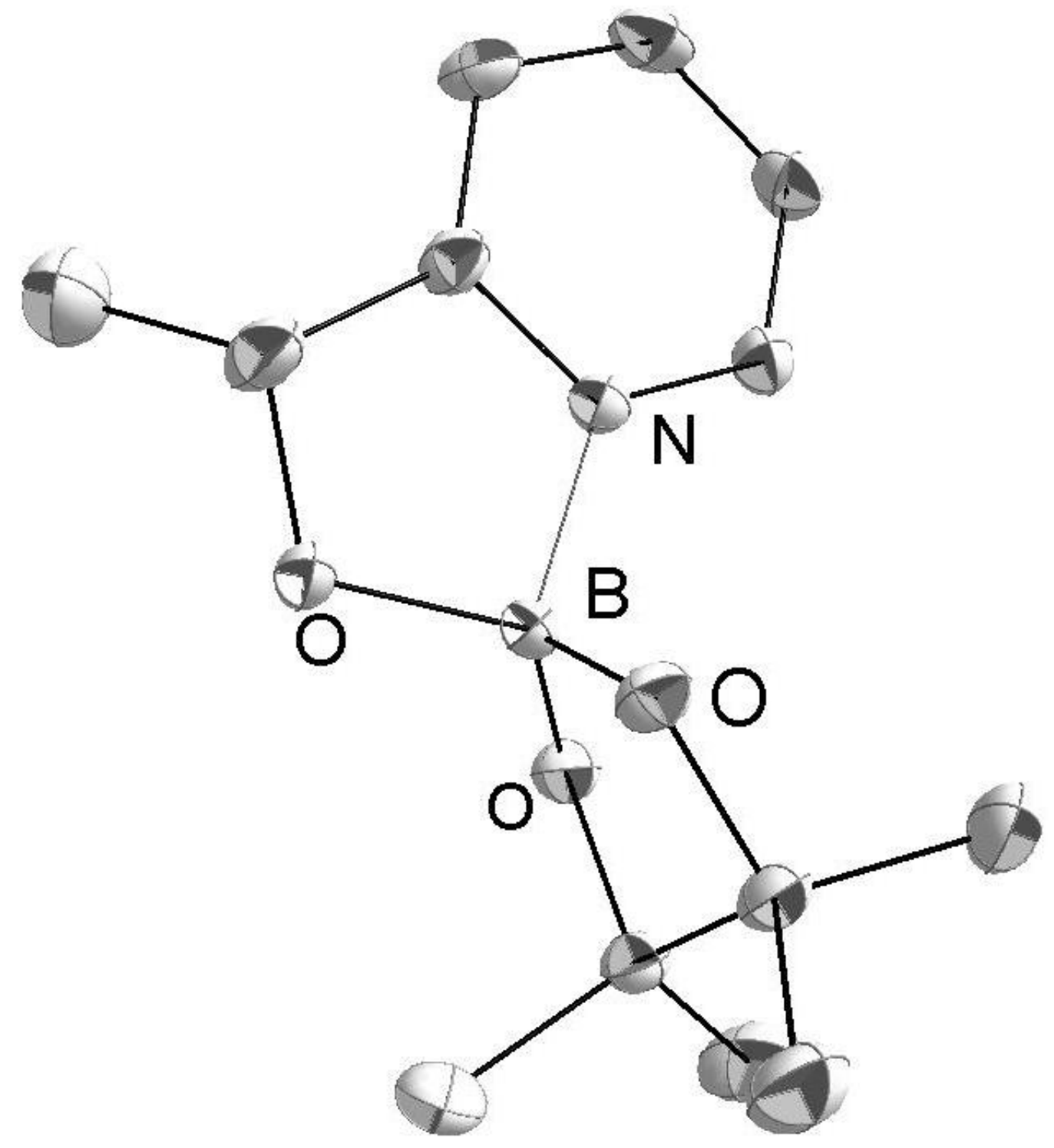

Figure S2. ORTEP plot of the molecular structure of 2t. Thermal ellipsoids are drawn at the $35 \%$ probability level. $\mathrm{H}$ atoms are omitted for clarity, except for the $\mathrm{Mg}-\mathrm{H}$ bridges. 
Table S1. Crystal data and structure refinement of complex $\mathbf{2}$ and $\mathbf{2 t}$.

\begin{tabular}{|c|c|c|}
\hline & 2 & $2 t$ \\
\hline Empirical formula & $\mathrm{C}_{70} \mathrm{H}_{84} \mathrm{Mg}_{2} \mathrm{~N}_{4} \mathrm{P}_{2}$ & $\mathrm{C}_{13} \mathrm{H}_{20} \mathrm{BNO}_{3}$ \\
\hline Crystal colour & Colorless & Yellow \\
\hline formula weight & 545.99 & 249.11 \\
\hline Crystal system & Triclinic & Monoclinic \\
\hline space group & $\mathrm{P}-1$ & $\mathrm{P} 2(1) / \mathrm{c}$ \\
\hline$a(\AA)$ & $12.0936(17)$ & 14.921 \\
\hline$b(\AA)$ & $12.3525(17)$ & 13.2590 \\
\hline$c(\AA)$ & $12.8290(18)$ & 14.2810 \\
\hline$\alpha(\operatorname{deg})$ & $66.390(2)$ & 90 \\
\hline$\beta(\mathrm{deg})$ & $63.998(2)$ & 103.27 \\
\hline$\gamma(\operatorname{deg})$ & $89.493(3)$ & 90 \\
\hline$V\left(\AA^{3}\right)$ & $1545.6(4)$ & 2749.88 \\
\hline$Z$ & 2 & 8 \\
\hline Dcalcd $\left(\mathrm{g} / \mathrm{cm}^{3}\right)$ & 1.173 & 1.203 \\
\hline$\mu\left(\mathrm{mm}^{-1}\right)$ & 0.135 & 0.083 \\
\hline $\mathrm{F}(000)$ & 586.0 & 1072.0 \\
\hline$\theta$ range, $\left({ }^{\circ}\right)$ & $1.84-26.40$ & $2.72-22.29$ \\
\hline No. of reflns collected & 8949 & 15273 \\
\hline No. of unique reflns $(I>2 \sigma(I))$ & 3010 & 5614 \\
\hline No. of params refnd & 363 & 335 \\
\hline Goodness of fit & 0.992 & 1.029 \\
\hline \multirow{2}{*}{ Final $R, R_{\mathrm{w}}(\mathrm{I}>2 \sigma(\mathrm{I}))$} & 0.0772 & 0.0795 \\
\hline & 0.2376 & 0.1951 \\
\hline
\end{tabular}




\subsection{Characterization Data for Hydroboration Products}

Table S2 Mg-catalyzed hydroboration of pyridines

\begin{tabular}{|c|c|c|c|c|}
\hline Entry & Temperature $\left({ }^{\circ} \mathrm{C}\right)$ & Yield $(\%)$ & Time $(\mathrm{h})$ & TOF $\left(h^{-1}\right)$ \\
\hline Pyridine (py) & 25 & 29.2 & 1 & 12.1 \\
\hline Pyridine (py) & 70 & 26.9 & 0.1 & 134.5 \\
\hline 2-Ac-(py) ${ }^{a}$ & 25 & $>99$ & $<2 \min$ & $>3000$ \\
\hline 3-Me-(py) & 25 & 33.3 & 1 & 12.6 \\
\hline 3-Ph-(py) & 25 & 89.3 & 1 & 44.6 \\
\hline 3-F-(py) & 25 & 65.7 & 0.58 & 55.8 \\
\hline 3-Cl-(py) & 25 & 81.9 & 0.28 & 183 \\
\hline 3-Br-(py) & 25 & 82 & 0.26 & 250 \\
\hline 3-I-(py) & 25 & 98 & 0.24 & 392 \\
\hline 3-OMe-(py) & 25 & 70.3 & 9 & 5.6 \\
\hline 3-MF-(py $)^{b}$ & 25 & $>99$ & $<2 \min$ & $>1500$ \\
\hline 3-СНО-(py) ${ }^{c}$ & 25 & $>99$ & $<2 \min$ & $>30000$ \\
\hline 4-Me-(py) & 25 & 38.0 & 6 & 3.1 \\
\hline 4-ph-(py) & 25 & 80.6 & 8 & 5.0 \\
\hline 4-MF-(py) $)^{b}$ & 25 & $>99$ & $<2 \min$ & $>1500$ \\
\hline pyrazine & 25 & $>99$ & $<2 \min$ & $>1500$ \\
\hline pyrimidine & 25 & 98 & 20 & 4.2 \\
\hline quinoline & 25 & $>99$ & $<2 \min$ & $>1500$ \\
\hline isoquinoline & 25 & $>99$ & $<2 \min$ & $>1500$ \\
\hline 3-Me-quinoline & 25 & $>99$ & $2 \min$ & 1500 \\
\hline 1-Me-isoquinoline & 60 & 98 & 24 & 2.0 \\
\hline 3-Me-isoquinoline & 25 & $>99$ & 1 & 50 \\
\hline 2,5-diMe-pyrazine & 60 & 98 & 6 & 8.1 \\
\hline quinoxaline & 25 & $>99$ & 6 & 8.3 \\
\hline
\end{tabular}

Reaction conditions: Pyridine derivative (0.189 mmol), HBpin (0.189 mmol), [1] (2 mol\%, 3.77 $\left.\times 10^{-3} \mathrm{mmol}\right), \mathrm{Ph}_{3} \mathrm{SiMe}\left(20 \times 10^{-3} \mathrm{mmol}\right)$ in $0.5 \mathrm{~mL} \mathrm{C}_{6} \mathrm{D}_{6}$ at $25^{\circ} \mathrm{C}$. Turnover frequencies (TOF $\left(\mathrm{h}^{-1}\right)=$ [product $\left.][\mathbf{1}]^{-1} \mathrm{~h}^{-1}\right)$ by ${ }^{1} \mathrm{H}$ NMR analysis with $\mathrm{Ph}_{3}$ SiMe internal standard. ${ }^{a} \mathrm{Ac}=$ acetyl, [1 $]_{0}$ $=1 \mathrm{~mol} \% ;{ }^{b} \mathrm{MF}=$ methyl formate $;{ }^{c} \mathrm{CHO}=$ aldehyde, $[\mathbf{1}]_{0}=0.1 \mathrm{~mol} \%$; 


\subsection{Procedure for Typical NMR-Scale Catalytic Reaction}

In glovebox, complex $1(4.2 \mathrm{mg}, 3.77 \mu \mathrm{mol})$ and triphenylmethylsilane (5.5 mg, $20 \mu \mathrm{mol})$ were added to a NMR tube with $0.5 \mathrm{~mL}$ of $\mathrm{C}_{6} \mathrm{D}_{6}$ and then put into refrigerator at $-35^{\circ} \mathrm{C}$ before use. Pyridine $(0.189 \mathrm{mmol}, 16 \mu \mathrm{L})$ and $\mathrm{HB}(0.189 \mathrm{mmol}, 29 \mu \mathrm{L})$ were quickly transferred to the NMR tube through micro-injector and then shaken just before NMR measurement. For each sample, a timer was started when $\mathrm{C}_{6} \mathrm{D}_{6}$ melting and the mixture turned to clean solution in NMR tube. Regular ${ }^{1} \mathrm{H}$ NMR spectra were obtained every 2 minutes. The related concentrations of pyridines and products were determined relative to the intensity of the internal standard resonance plotted versus time. 
1.4.1 Spectroscopic Characterization of ligands, complexes and 2a-2w

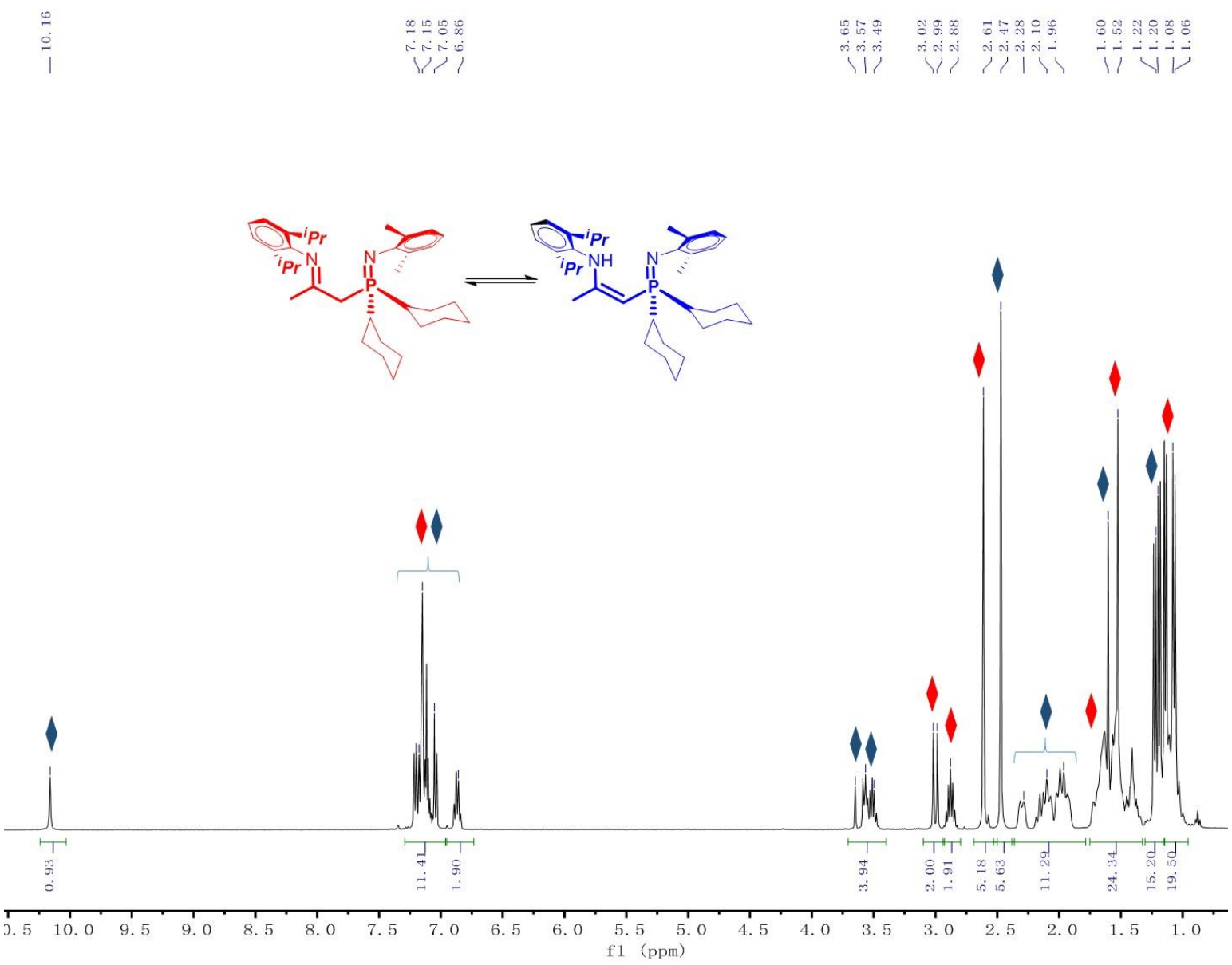

Figure S3. ${ }^{1} \mathrm{H}-\mathrm{NMR}$ spectrum (400 MHz, $\mathrm{CDCl}_{3}$ ) of $\mathbf{H L 1}$. 


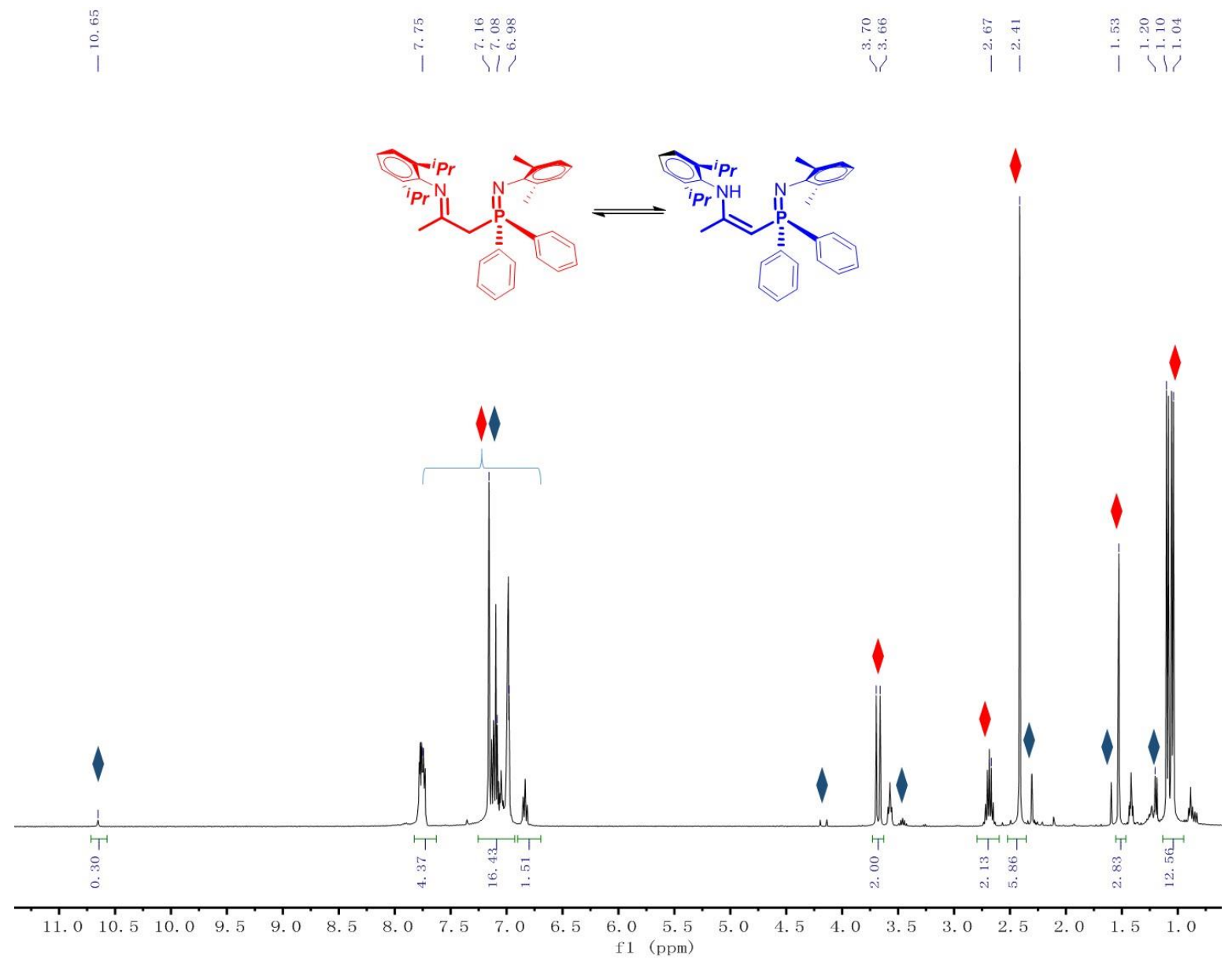

Figure S4. ${ }^{1} \mathrm{H}-\mathrm{NMR}$ spectrum $\left(400 \mathrm{MHz}, \mathrm{CDCl}_{3}\right)$ of $\mathbf{H L 2}$. 

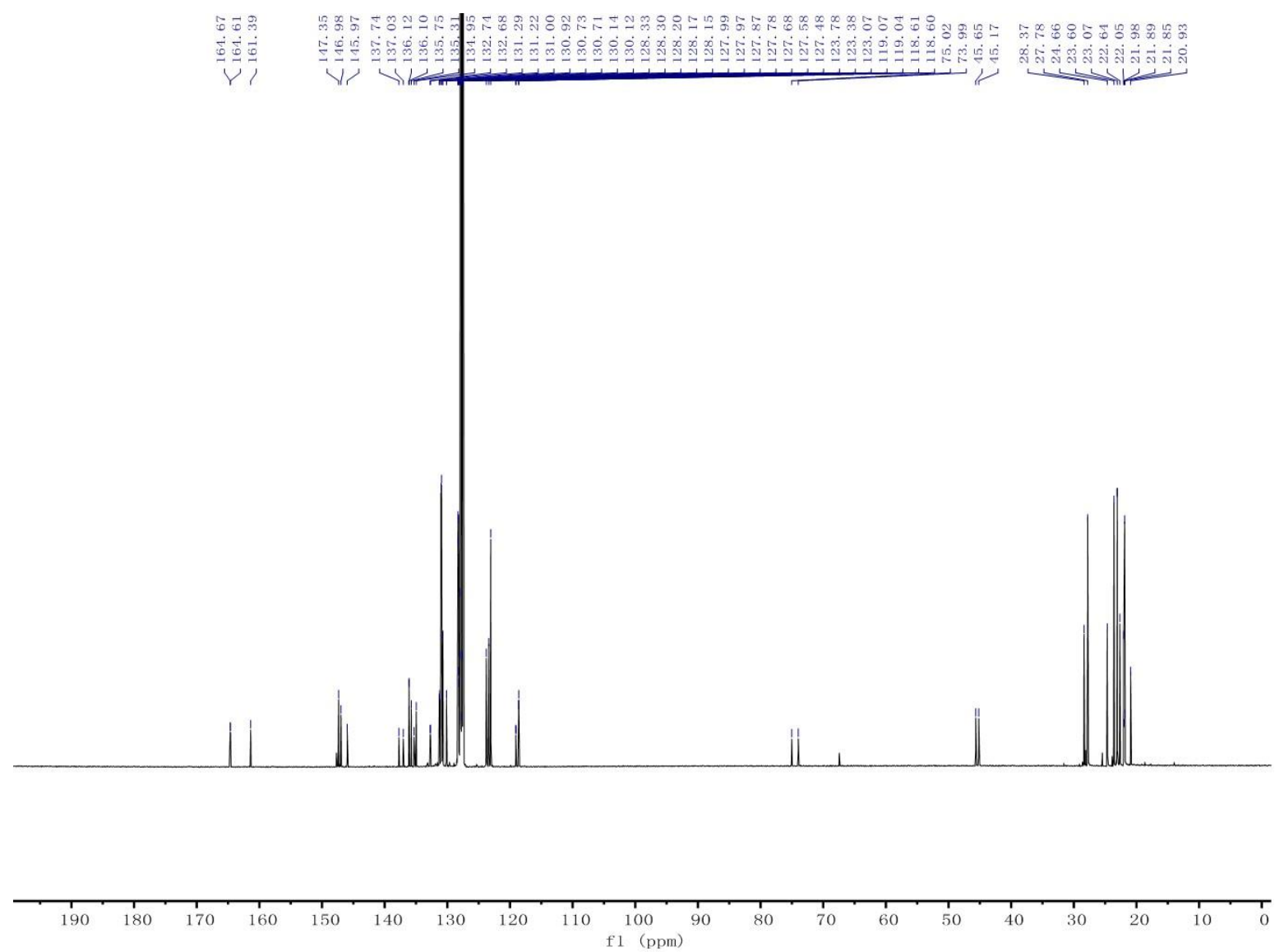

Figure S5. ${ }^{13} \mathrm{C}$-NMR spectrum $\left(100 \mathrm{MHz}, \mathrm{CDCl}_{3}\right)$ of $\mathbf{H L 2}$. 
$\frac{5}{1}$

$=0$
11

$\stackrel{\infty}{\infty}$

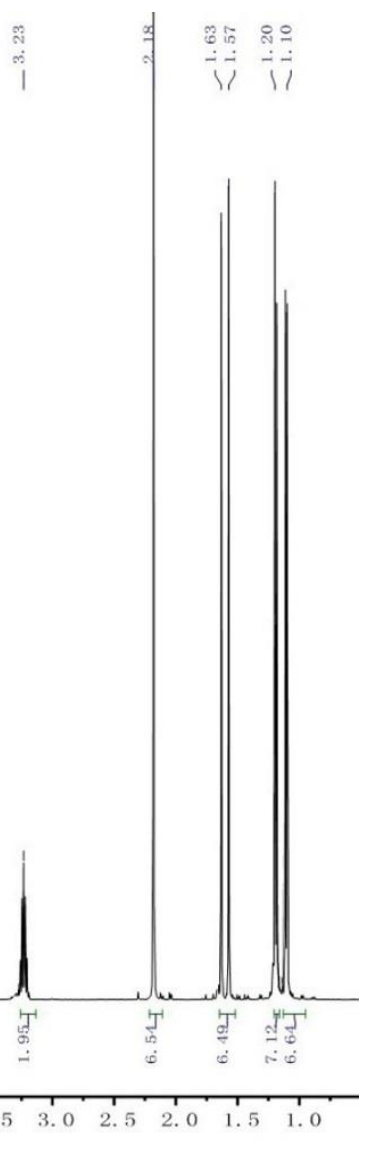

Figure S6. ${ }^{1} \mathrm{H}-\mathrm{NMR}$ spectrum $\left(400 \mathrm{MHz}, \mathrm{CDCl}_{3}\right)$ of $\mathbf{H L 3}$.

$15 / 86$ 

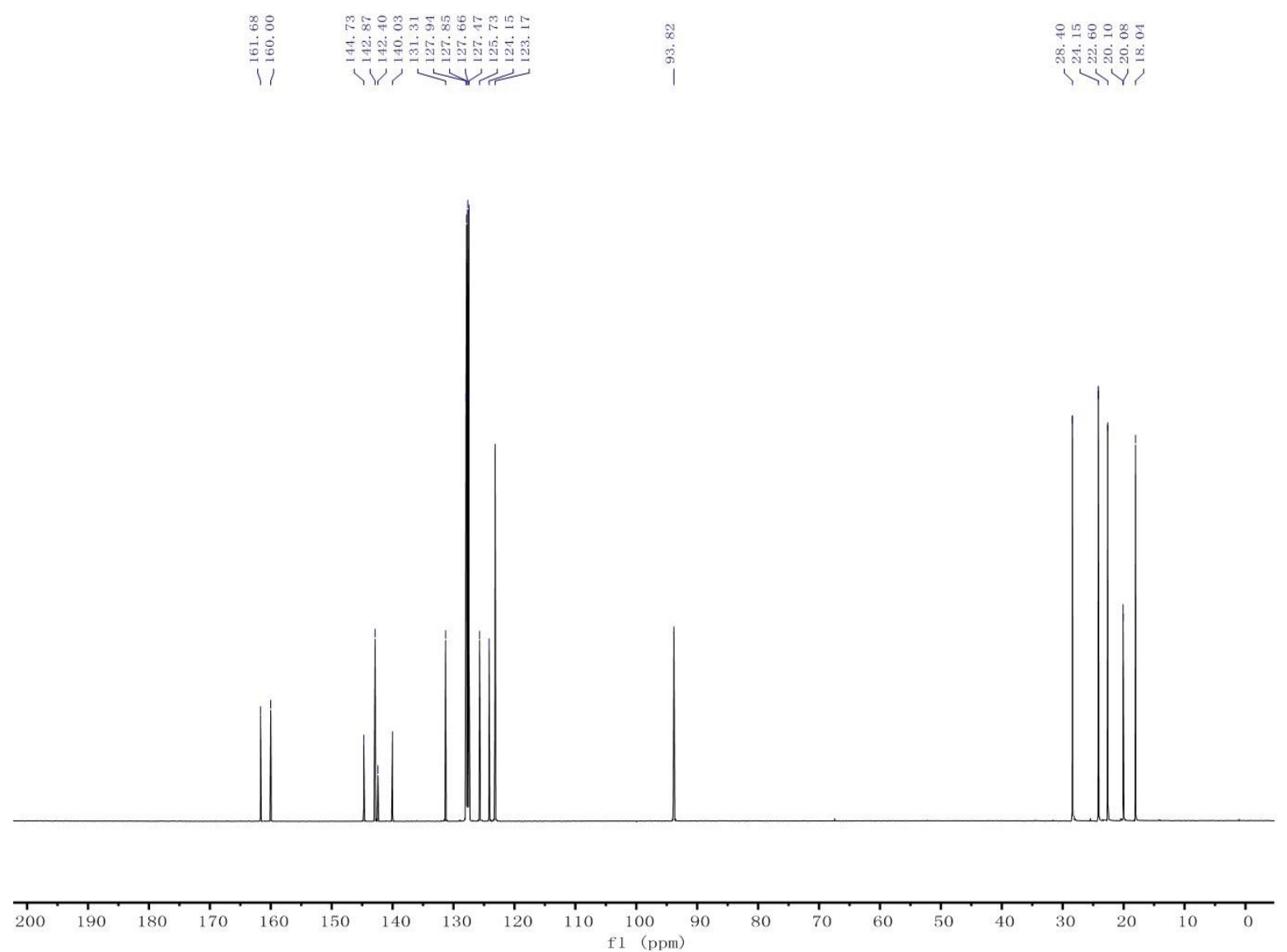

Figure S7. ${ }^{13} \mathrm{C}-\mathrm{NMR}$ spectrum $\left(100 \mathrm{MHz}, \mathrm{CDCl}_{3}\right)$ of $\mathbf{H L 3}$. 


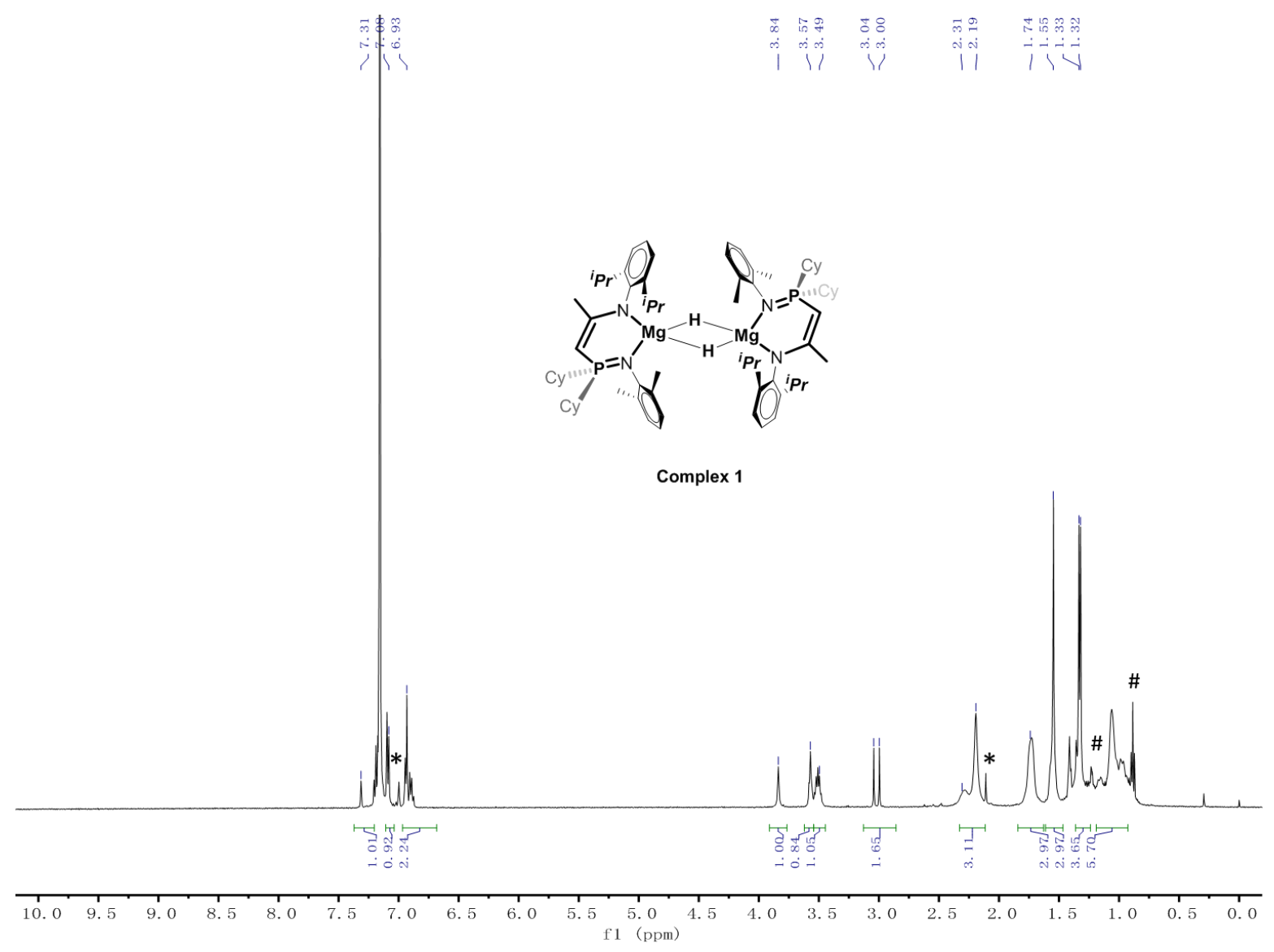

Figure S8. ${ }^{1} \mathrm{H}-\mathrm{NMR}$ spectrum $\left(400 \mathrm{MHz}, \mathrm{C}_{6} \mathrm{D}_{6}\right)$ of Complex 1. (* hexane; ${ }^{*}$ toluene) 


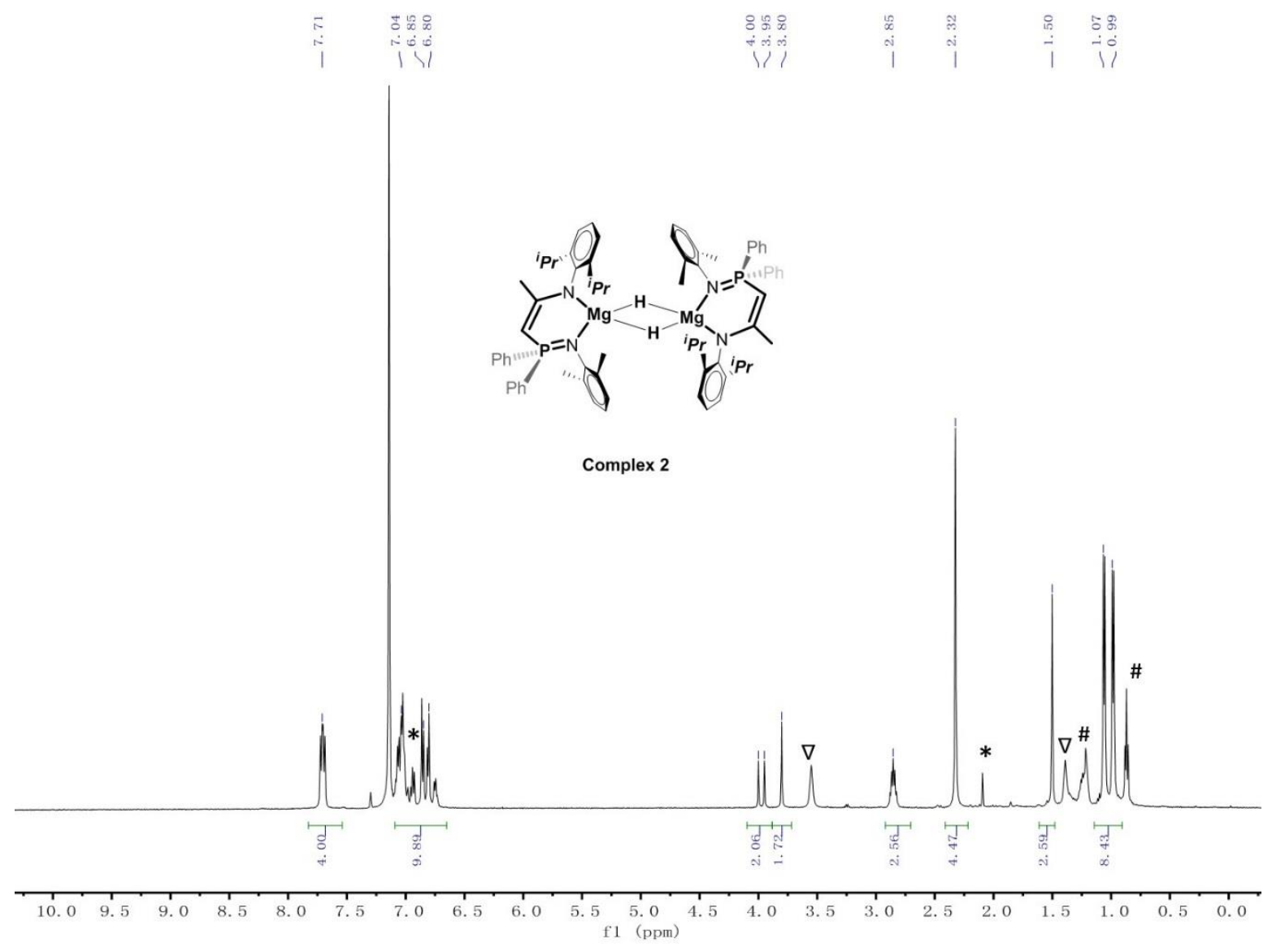

Figure S9. ${ }^{1} \mathrm{H}-\mathrm{NMR}$ spectrum $\left(400 \mathrm{MHz}, \mathrm{C}_{6} \mathrm{D}_{6}\right)$ of Complex 2. (* hexane; ${ }^{*}$ toluene; $\left.{ }^{\nabla} \mathrm{THF}\right)$ 

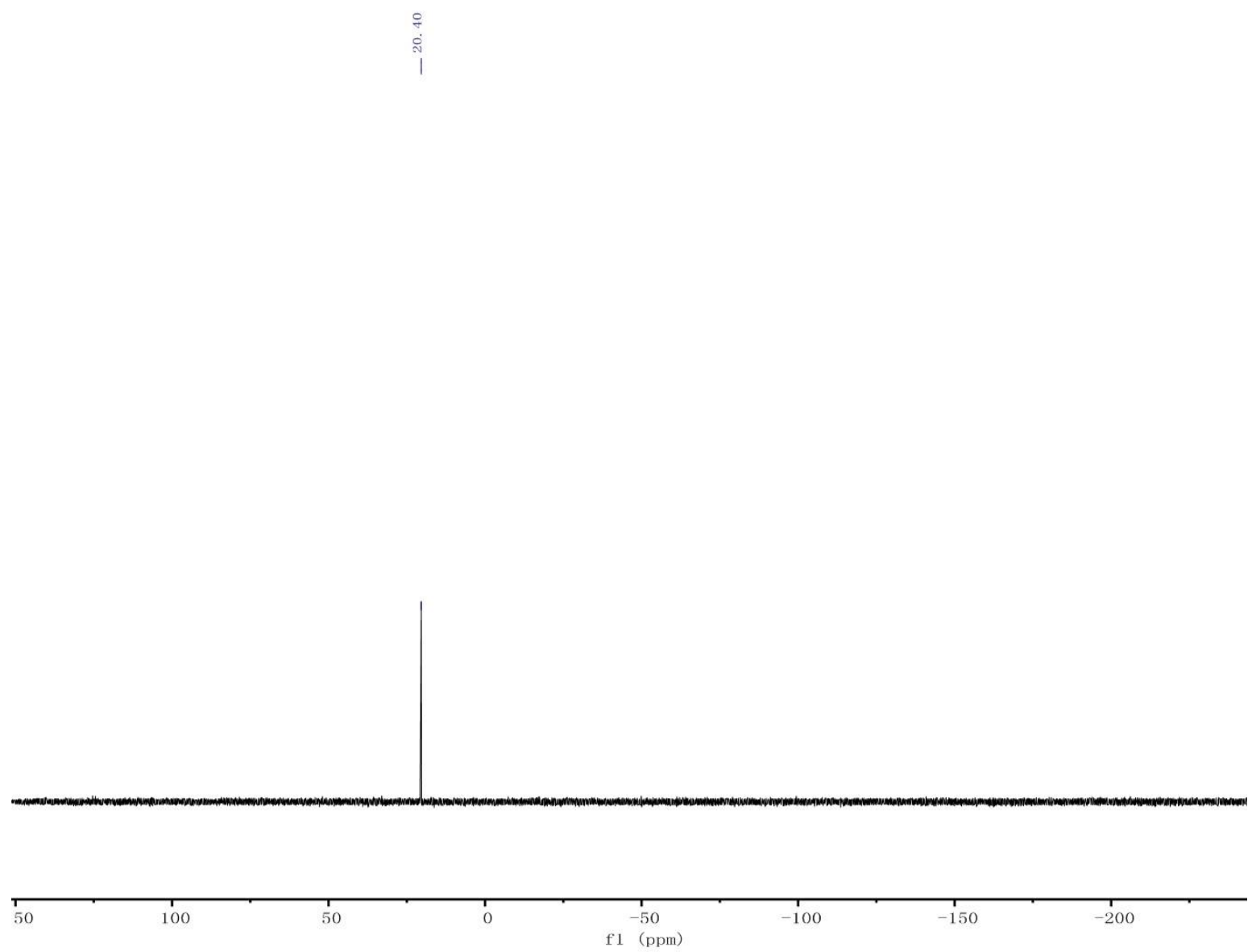

Figure S10. ${ }^{31} \mathrm{P}-\mathrm{NMR}$ spectrum $\left(162 \mathrm{MHz}, \mathrm{C}_{6} \mathrm{D}_{6}\right)$ of Complex 2. 

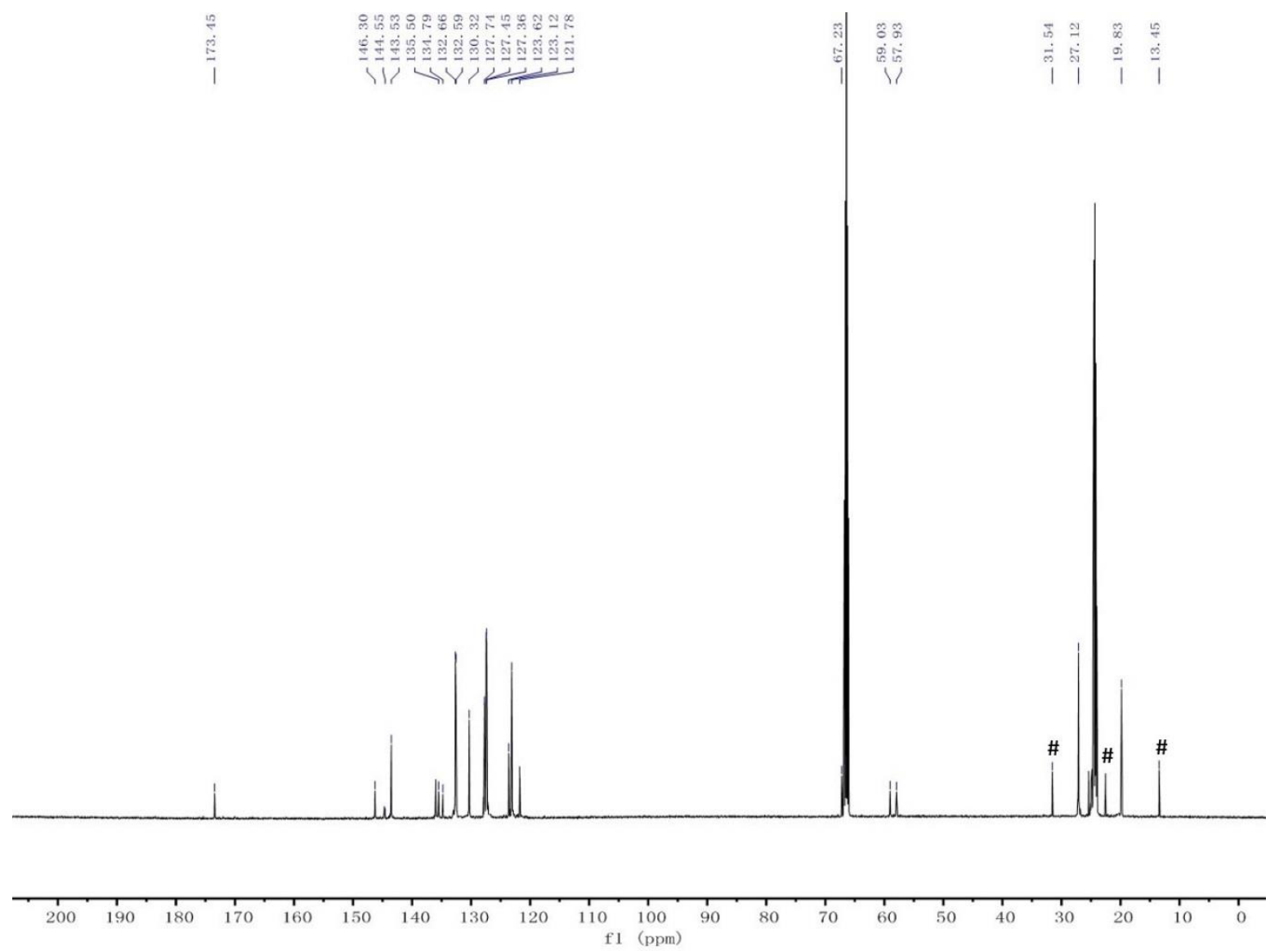

Figure S11. ${ }^{13} \mathrm{C}-\mathrm{NMR}$ spectrum (100 MHz, $d{ }^{8}$-THF) of Complex 2. (" hexane) 


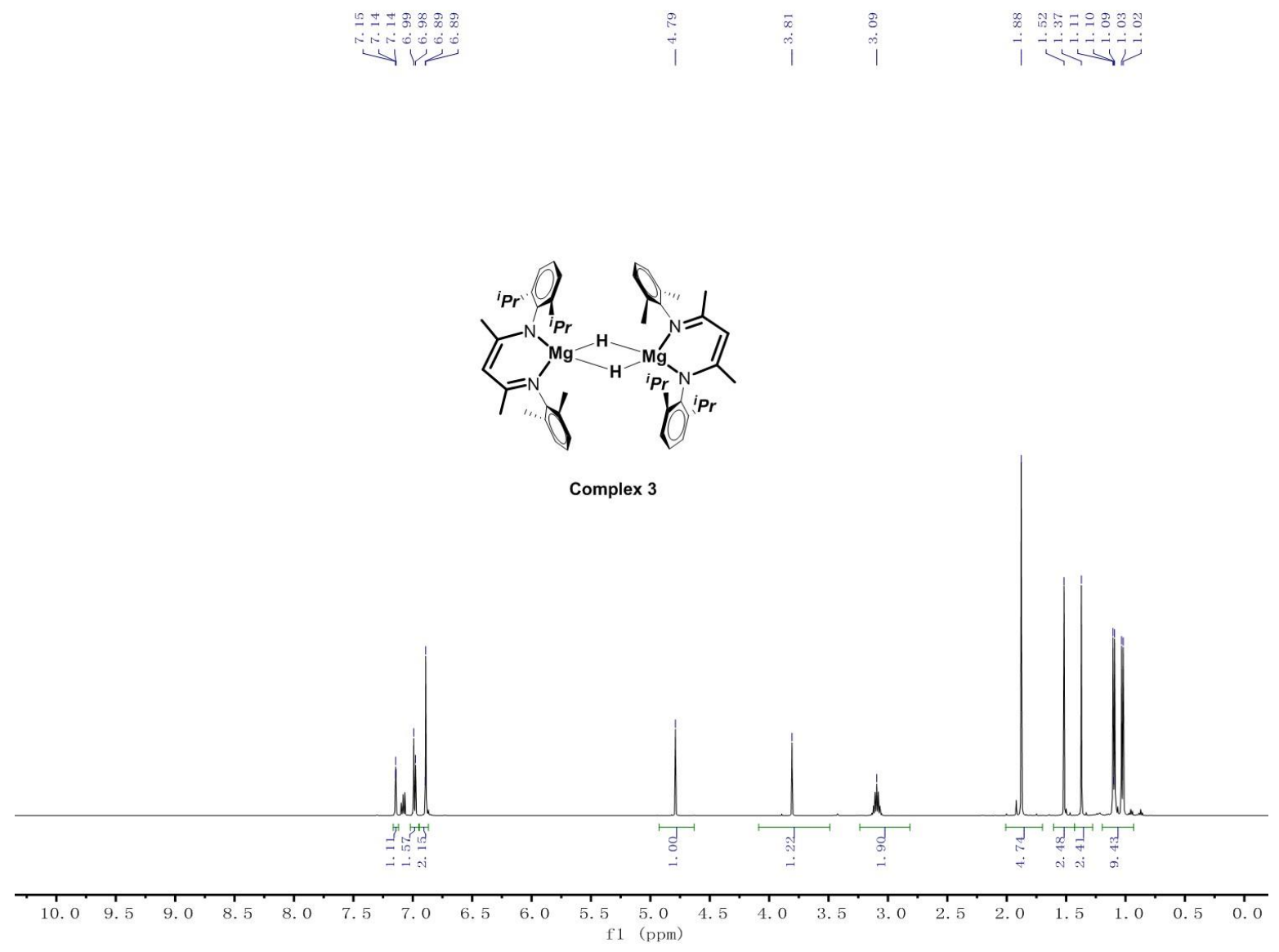

Figure S12. ${ }^{1} \mathrm{H}-\mathrm{NMR}$ spectrum $\left(400 \mathrm{MHz}, \mathrm{C}_{6} \mathrm{D}_{6}\right)$ of Complex 3. 


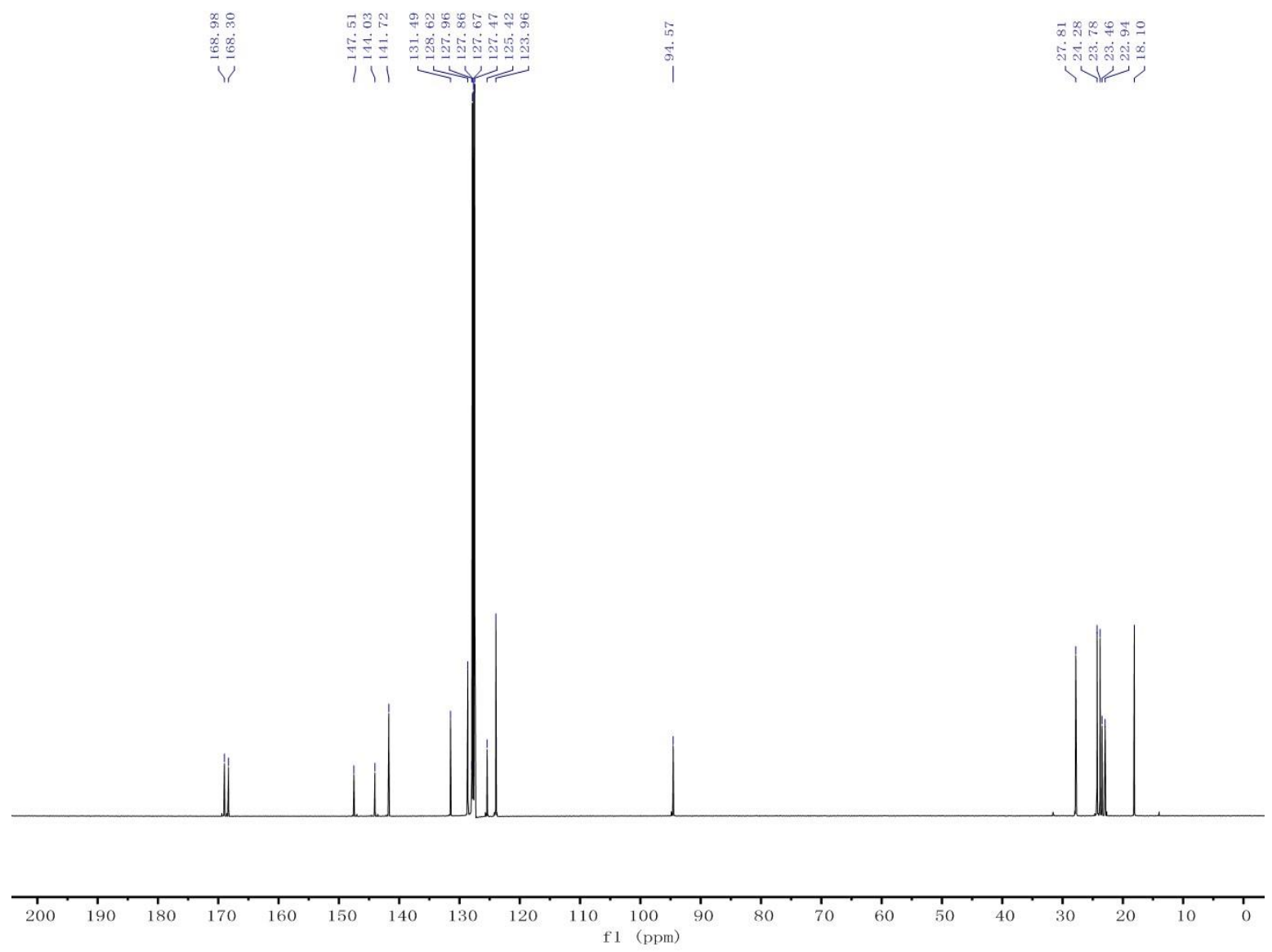

Figure S13. ${ }^{13} \mathrm{C}-\mathrm{NMR}$ spectrum $\left(100 \mathrm{MHz}, \mathrm{C}_{6} \mathrm{D}_{6}\right)$ of Complex 3. 


\section{1-(4,4,5,5-Tetramethyl-1,3,2-dioxaborolan-2-yl)-1,2-dihydropyridine (2a):}

Light yellow liquid (173 mg, 84\%):

${ }^{1} \mathrm{H}$ NMR (500 MHz, $\left.\mathrm{C}_{6} \mathrm{D}_{6}\right): \delta=6.71(\mathrm{~d}, J=5.0 \mathrm{~Hz}, 1 \mathrm{H}), 5.78(\mathrm{~d}, J=5.0 \mathrm{~Hz}, 1 \mathrm{H}), 5.09(\mathrm{~m}, 1 \mathrm{H})$, $4.15(\mathrm{~m}, 2 \mathrm{H}), 1.00(\mathrm{~s}, 12 \mathrm{H}) \mathrm{ppm}$.

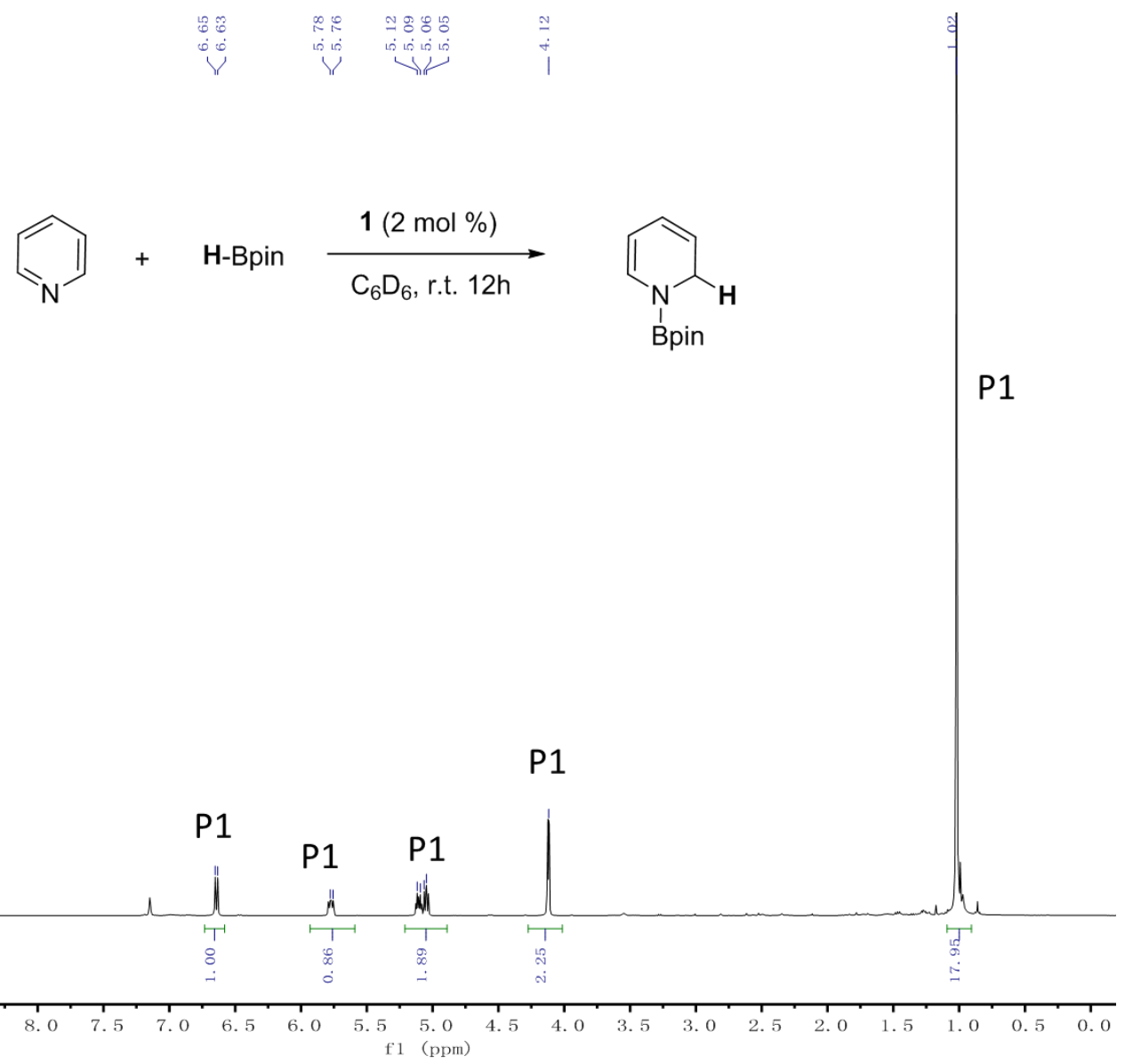

Figure S14. ${ }^{1} \mathrm{H}-\mathrm{NMR}$ spectrum $\left(500 \mathrm{MHz}, \mathrm{C}_{6} \mathrm{D}_{6}\right)$ of the product of 1,2-hydroboration of pyridine catalyzed by 1 . Assignment: $\mathrm{P} 1$ = predominant product; 


\section{4-methyl-1-(4,4,5,5-tetramethyl-1,3,2-dioxaborolan-2-yl)-1,2-dihydropyridine (2b):}

Colorless oil (198 mg, 90\%):

${ }^{1} \mathrm{H}$ NMR $\left(500 \mathrm{MHz}, \mathrm{C}_{6} \mathrm{D}_{6}\right): \delta=6.72(\mathrm{~d}, J=5.0 \mathrm{~Hz}, 1 \mathrm{H}), 4.97(\mathrm{~d}, J=5.0 \mathrm{~Hz}, 1 \mathrm{H}), 4.86(\mathrm{~m}, 1 \mathrm{H})$, $4.18(\mathrm{~m}, 2 \mathrm{H}), 1.57(\mathrm{~d}, J=5.0 \mathrm{~Hz}, 3 \mathrm{H}), 1.00(\mathrm{~s}, 12 \mathrm{H}) \mathrm{ppm}$.

${ }^{13} \mathrm{C}$ NMR (125 MHz, $\mathrm{C}_{6} \mathrm{D}_{6}$ ): 131.8, 131.4, 110.0, 106.5, 82.7, 42.6, 24.3, $20.3 \mathrm{ppm}$.

Anal. Calc. for $\mathrm{C}_{12} \mathrm{H}_{20} \mathrm{BNO}_{2}$ : C, 65.19; H, 9.12; N, 6.33. Found: C, 65.12; H, 9.01; N, 6.29.

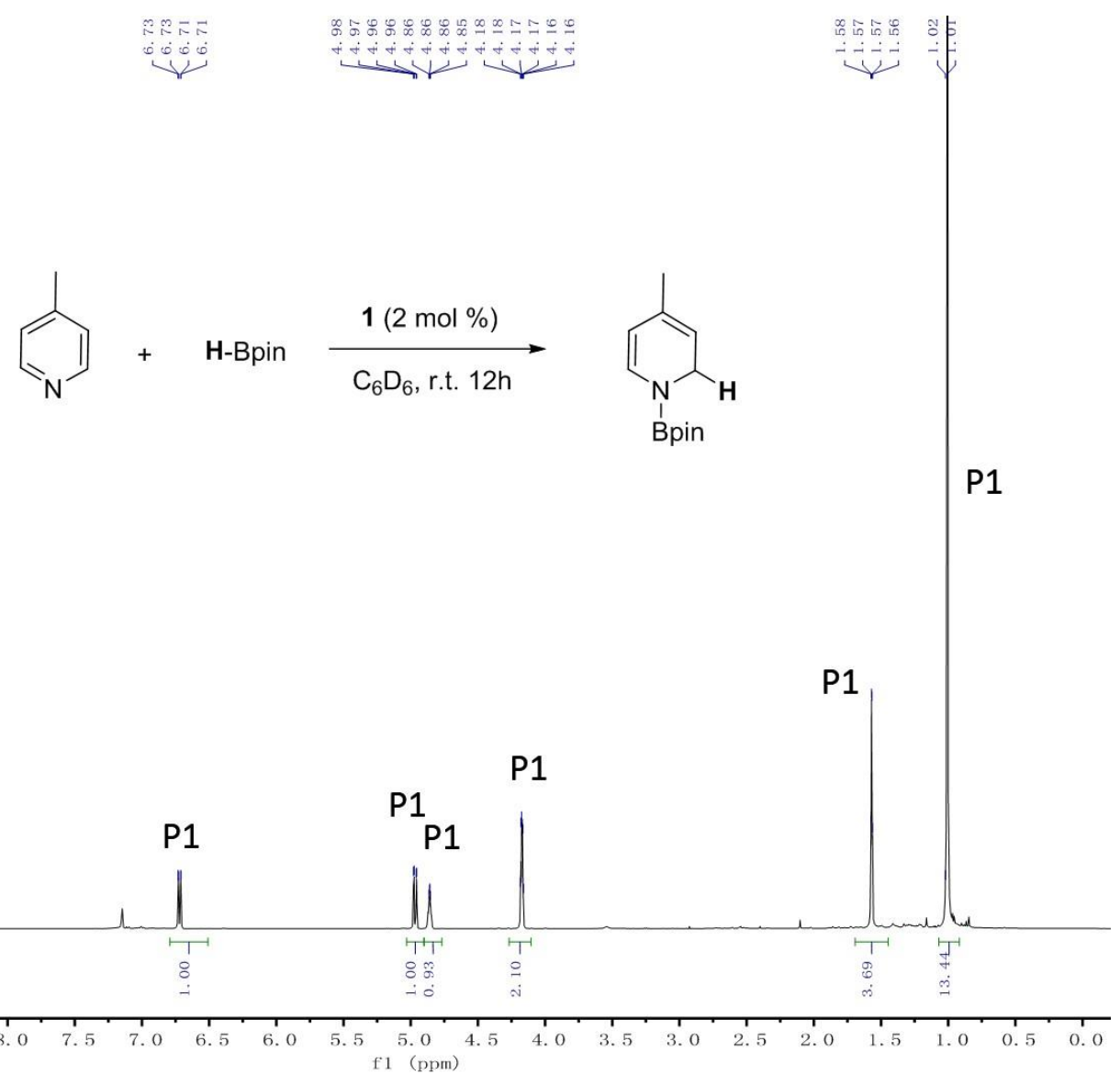

Figure S15. ${ }^{1} \mathrm{H}-\mathrm{NMR}$ spectrum $\left(500 \mathrm{MHz}, \mathrm{C}_{6} \mathrm{D}_{6}\right)$ of the product of 1,2-hydroboration of 4methylpyridine catalyzed by 1 . Assignment: P1 = predominant product of 4-methyl-1,2dihydropyridine. 


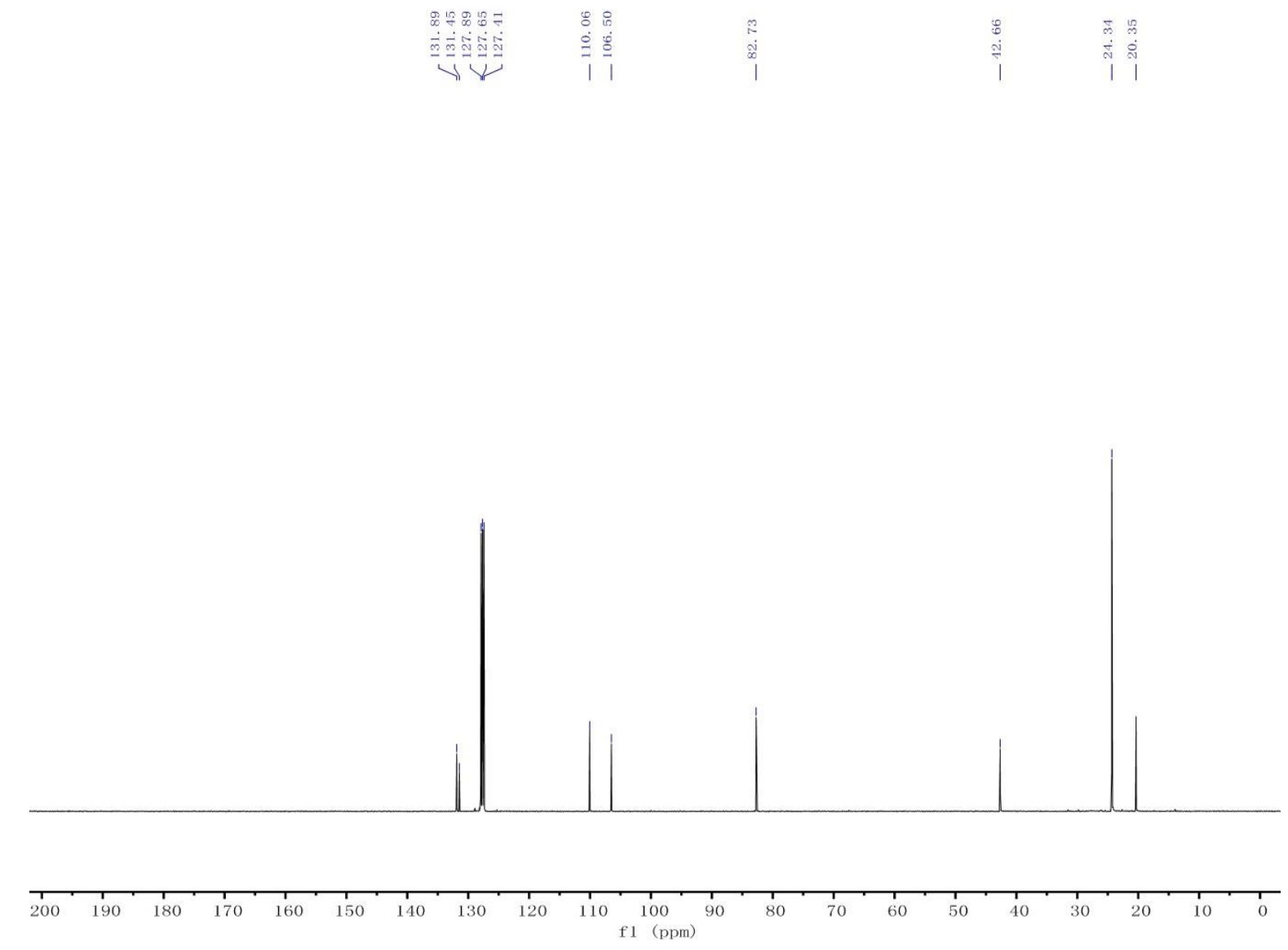

Figure S16. ${ }^{13} \mathrm{C}-\mathrm{NMR}$ spectrum $\left(125 \mathrm{MHz}, \mathrm{C}_{6} \mathrm{D}_{6}\right)$ of the product of 1,2-hydroboration of 4methylpyridine catalyzed by $\mathbf{1}$. 


\section{4-phenyl-1-(4,4,5,5-tetramethyl-1,3,2-dioxaborolan-2-yl)-1,2-dihydropyridine (2c):}

Colorless solid (257 mg, 91\%):

${ }^{1} \mathrm{H}$ NMR $\left(500 \mathrm{MHz}, \mathrm{C}_{6} \mathrm{D}_{6}\right): \delta=7.32(\mathrm{~d}, J=10.0 \mathrm{~Hz}, 1 \mathrm{H}), 7.14-7.06(\mathrm{~m}, 4 \mathrm{H}), 6.87(\mathrm{~d}, J=5.0$ $\mathrm{Hz}, 1 \mathrm{H}), 5.32$ (dd, $J=5.0 \mathrm{~Hz}, 1 \mathrm{H}), 5.34(\mathrm{~m}, 1 \mathrm{H}), 4.28(\mathrm{~d}, J=5.0 \mathrm{~Hz}, 2 \mathrm{H}), 1.02$ (s, 12H) ppm. ${ }^{13} \mathrm{C}$ NMR (125 MHz, $\left.\mathrm{C}_{6} \mathrm{D}_{6}\right): 139.8,135.8,133.2,128.2,126.9,125.5,110.9,104.2,82.9,42.8$, 24.3.

Anal. Calc. for $\mathrm{C}_{17} \mathrm{H}_{22} \mathrm{BNO}_{2}$ : C, 72.11; H, 7.83; N, 4.95. Found: C, 72.10; H, 7.78; N, 4.90.

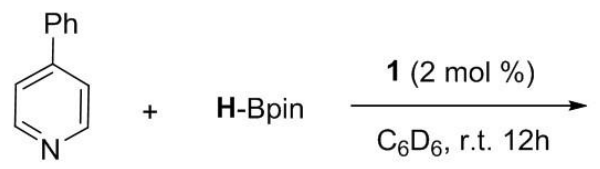<smiles>Brc1ccccc1</smiles>

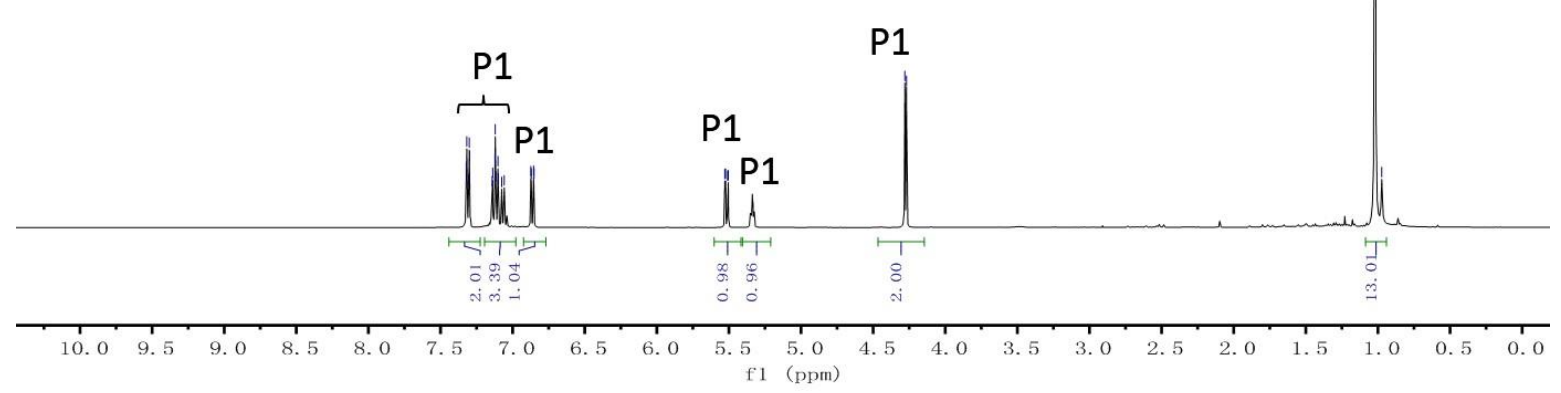

Figure S17. ${ }^{1} \mathrm{H}-\mathrm{NMR}$ spectrum $\left(500 \mathrm{MHz}, \mathrm{C}_{6} \mathrm{D}_{6}\right)$ of the product of 1,2-hydroboration of 4phenylpyridine catalyzed by 1 . Assignment: P1 = predominant product of 4-phenyl-1,2dihydropyridine. 


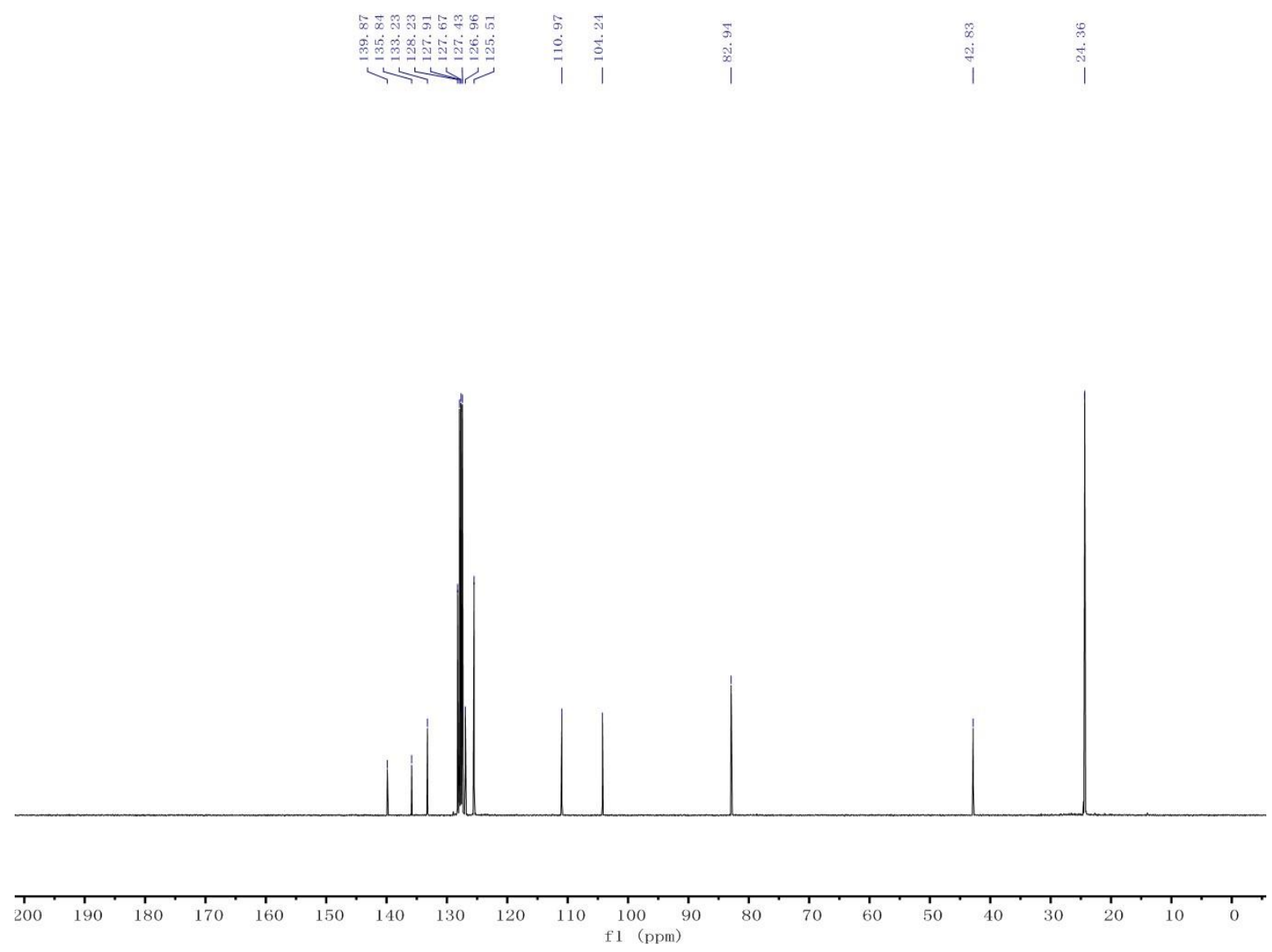

Figure S18. ${ }^{13} \mathrm{C}-\mathrm{NMR}$ spectrum $\left(125 \mathrm{MHz}, \mathrm{C}_{6} \mathrm{D}_{6}\right)$ of the product of 1,2-hydroboration of 4phenylpyridine catalyzed by $\mathbf{1}$. 


\section{1-(4,4,5,5-Tetramethyl-1,3,2-dioxaborolan-2-yl)-3-methyl-1,2-dihydropyridine (2d):}

Light yellow liquid (161 mg, 73\%):

${ }^{1} \mathrm{H}$ NMR $\left(500 \mathrm{MHz}, \mathrm{C}_{6} \mathrm{D}_{6}\right): \delta=6.42(\mathrm{~d}, J=10.0 \mathrm{~Hz}, 1 \mathrm{H}), 5.43(\mathrm{~m} 1 \mathrm{H}), 5.34(\mathrm{~m}, 1 \mathrm{H}), 5.06(\mathrm{dd}$, $\left.{ }^{3} J=10.0 \mathrm{~Hz},{ }^{4} J=10.0 \mathrm{~Hz}, 2 \mathrm{H}\right), 4.10$ (s, 2H), 1.42 (s, 3H), 1.03 (s, 12H) ppm.

${ }^{13} \mathrm{C}$ NMR (125 MHz, $\left.\mathrm{C}_{6} \mathrm{D}_{6}\right):$ 128.9, 124.3, 118.2, 82.7, 46.9, 24.3, $20.3 \mathrm{ppm}$.

Anal. Calc. for $\mathrm{C}_{12} \mathrm{H}_{20} \mathrm{BNO}_{2}$ : C, 65.19; H, 9.12; N, 6.33. Found: C, 65.17; H, 9.00; N, 6.31.

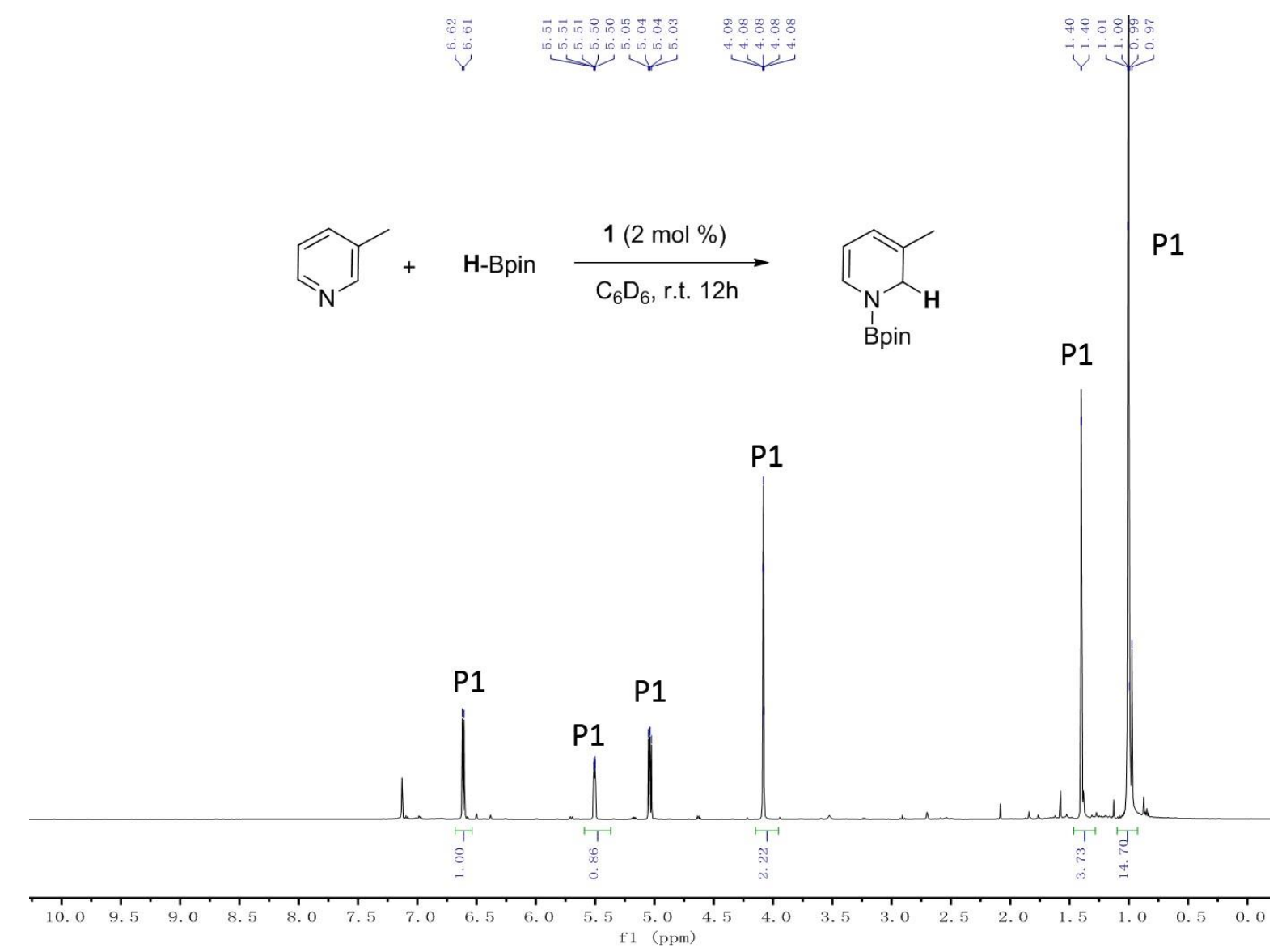

Figure S19. ${ }^{1} \mathrm{H}-\mathrm{NMR}$ spectrum $\left(500 \mathrm{MHz}, \mathrm{C}_{6} \mathrm{D}_{6}\right)$ of the product of 1,2-hydroboration of 3methylpyridine catalyzed by 1 . Assignment: P1 = predominant product of 3-methyl-1,2dihydropyridine. 


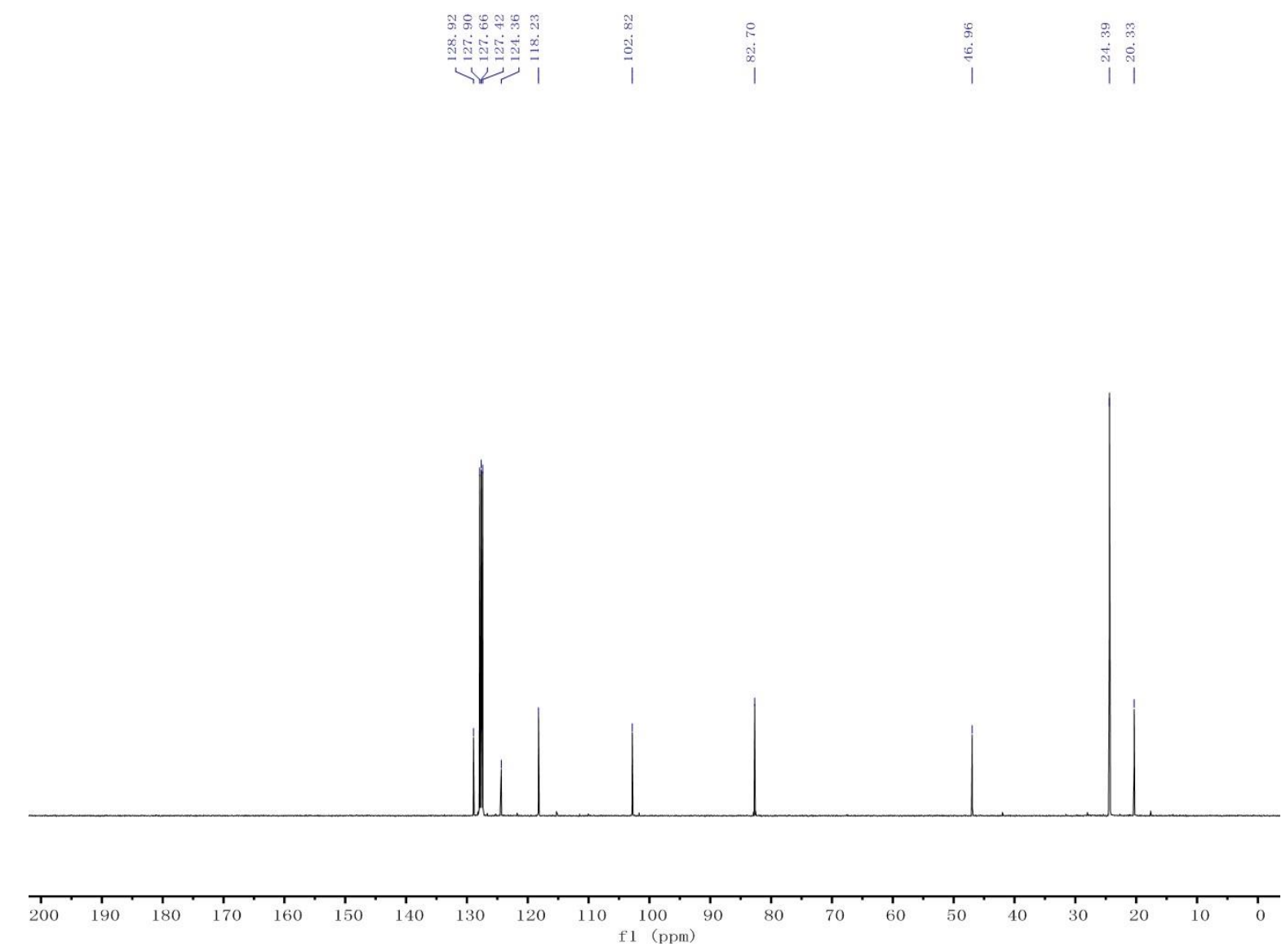

Figure S20. ${ }^{13} \mathrm{C}-\mathrm{NMR}$ spectrum $\left(125 \mathrm{MHz}, \mathrm{C}_{6} \mathrm{D}_{6}\right)$ of the product of 1,2-hydroboration of 3methylpyridine catalyzed by $\mathbf{1}$. 


\section{1-(4,4,5,5-Tetramethyl-1,3,2-dioxaborolan-2-yl)-3-phenyl-1,2-dihydropyridine (2e):}

Colorless solid (198 mg, 70\%):

${ }^{1} \mathrm{H}$ NMR $\left(500 \mathrm{MHz}, \mathrm{C}_{6} \mathrm{D}_{6}\right): \delta=7.25-6.99(\mathrm{~m}, 5 \mathrm{H}), 6.81(\mathrm{~d}, J=5.0 \mathrm{~Hz}, 1 \mathrm{H}), 6.27(\mathrm{~d}, J=5.0 \mathrm{~Hz}$, $1 \mathrm{H}), 5.27(\mathrm{t}, J=5.0 \mathrm{~Hz}, 1 \mathrm{H}), 4.62(\mathrm{~s}, 2 \mathrm{H}), 1.03(\mathrm{~s}, 12 \mathrm{H}) \mathrm{ppm}$.

${ }^{13} \mathrm{C}$ NMR (125 MHz, $\left.\mathrm{C}_{6} \mathrm{D}_{6}\right): 138.7,131.7,128.2,126.3,124.3,119.8,103.9,83.0,44.4,24.3$

ppm.

Anal. Calc. for $\mathrm{C}_{17} \mathrm{H}_{22} \mathrm{BNO}_{2}$ : C, 72.11; H, 7.83; N, 4.95. Found: C, 72.08; H, 7.76; N, 4.91.

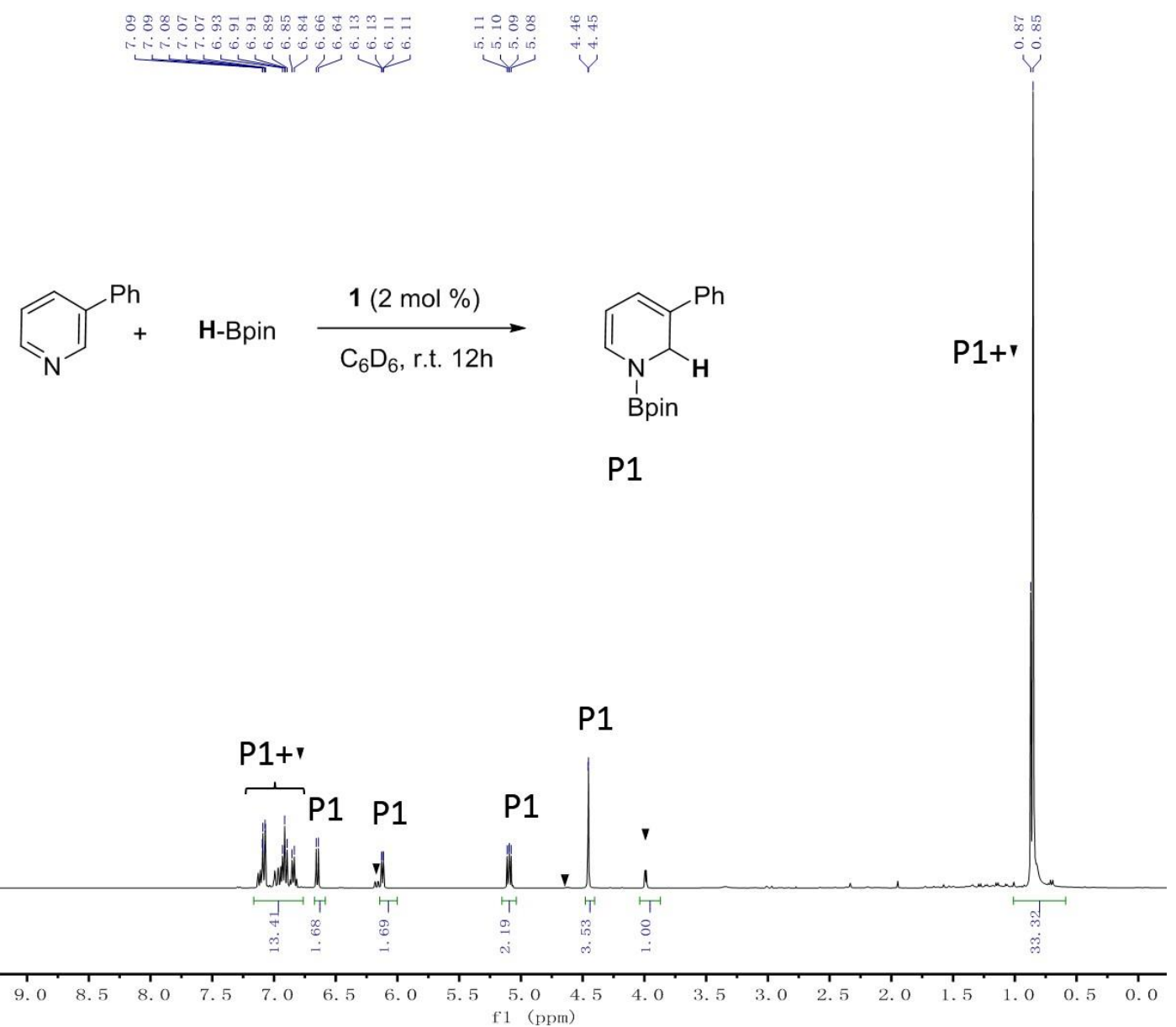

Figure S21. ${ }^{1} \mathrm{H}-\mathrm{NMR}$ spectrum $\left(500 \mathrm{MHz}, \mathrm{C}_{6} \mathrm{D}_{6}\right)$ of the product of 1,2-hydroboration of 3phenylpyridine catalyzed by 1 . Assignment: P1 = predominant product of 3-phenyl-1,2dihydropyridine; $\mathbf{7}$-phenyl-1,2-dihydropyridine. 


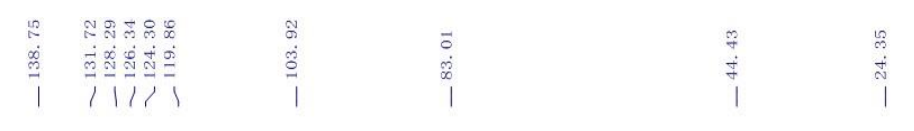
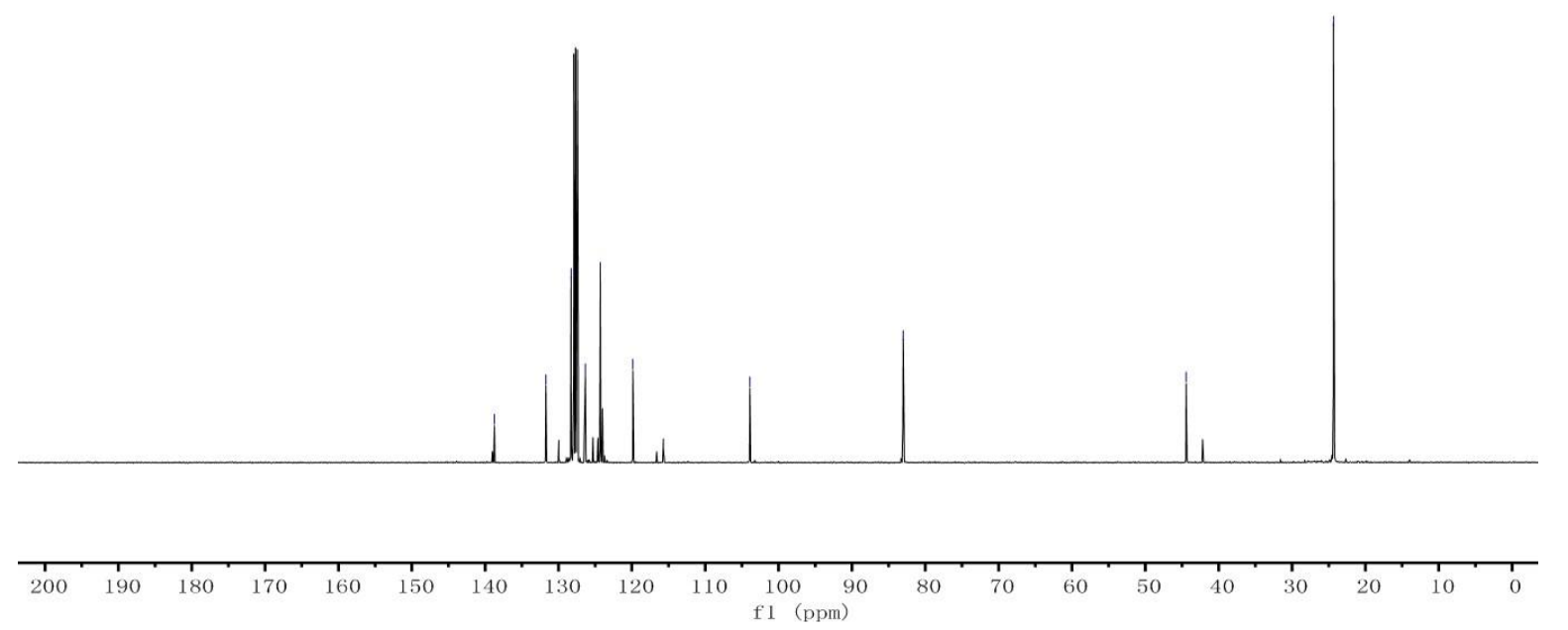

Figure S22. ${ }^{13} \mathrm{C}-\mathrm{NMR}$ spectrum $\left(125 \mathrm{MHz}, \mathrm{C}_{6} \mathrm{D}_{6}\right)$ of the product of 1,2-hydroboration of 3phenylpyridine catalyzed by $\mathbf{1}$. 


\section{1-(4,4,5,5-Tetramethyl-1,3,2-dioxaborolan-2-yl)-3-methoxy-1,2-dihydropyridine (2f):}

Colorless oil (215 mg, 91\%):

${ }^{1} \mathrm{H}$ NMR $\left(500 \mathrm{MHz}, \mathrm{C}_{6} \mathrm{D}_{6}\right): 6.52(\mathrm{~d}, J=10.0 \mathrm{~Hz}, 1 \mathrm{H}), 5.12(\mathrm{dd}, J=10.0 \mathrm{~Hz}, 1 \mathrm{H}), 4.74(\mathrm{~d}, J=$ $5.0 \mathrm{~Hz}, 1 \mathrm{H}), 4.33(\mathrm{~s}, 2 \mathrm{H}), 1.00(\mathrm{~s}, 12 \mathrm{H}) \mathrm{ppm}$.

${ }^{13} \mathrm{C}$ NMR (125 MHz, $\left.\mathrm{C}_{6} \mathrm{D}_{6}\right): 148.9,124.5,102.4,91.2,82.7,53.8,45.0,24.3 \mathrm{ppm}$.

Anal. Calc. for $\mathrm{C}_{12} \mathrm{H}_{20} \mathrm{BNO}_{3}$ : C, 60.79; H, 8.50; N, 5.91. Found: C, 60.72; H, 8.39; N, 5.78.

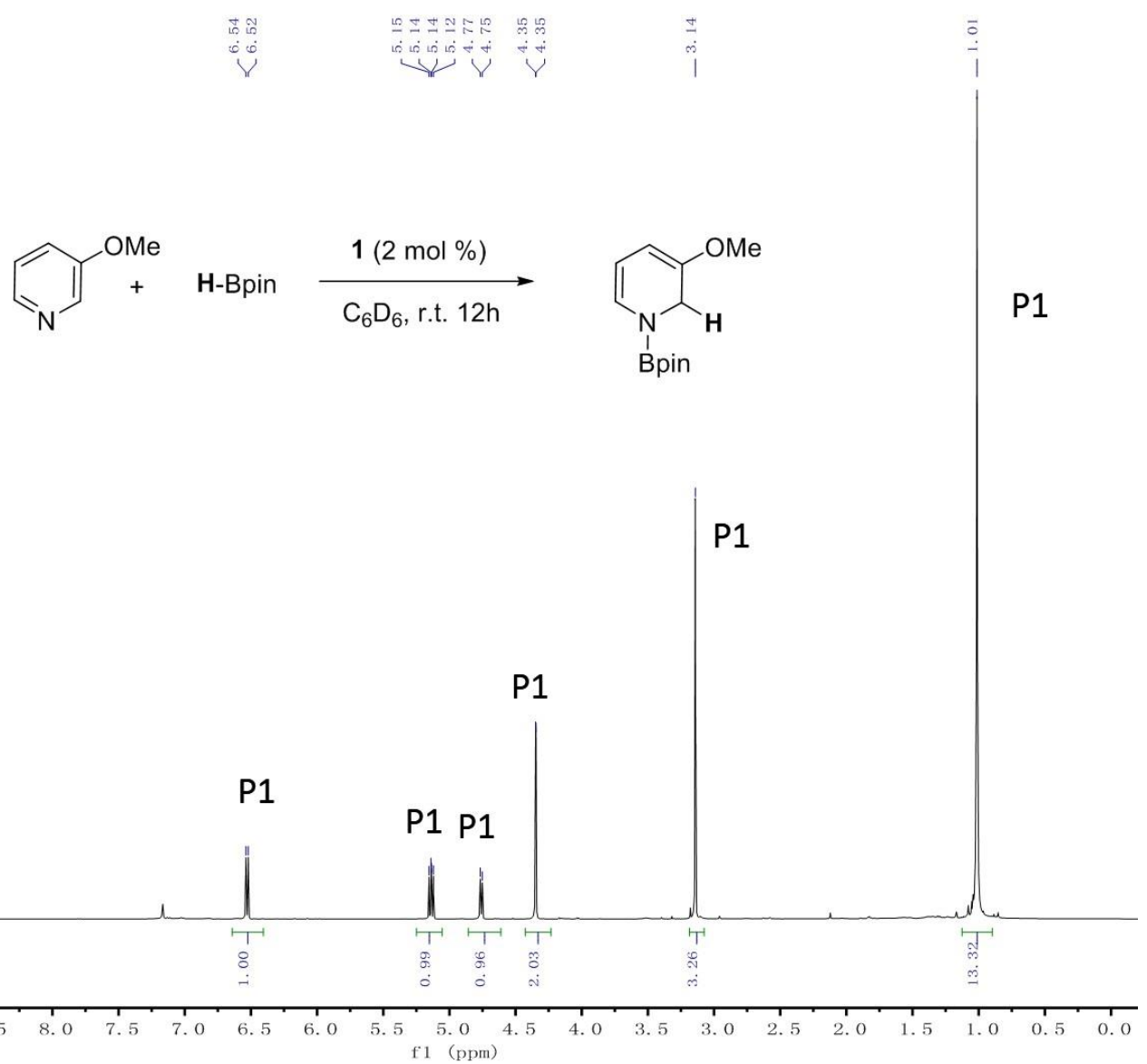

Figure S23. ${ }^{1} \mathrm{H}-\mathrm{NMR}$ spectrum $\left(500 \mathrm{MHz}, \mathrm{C}_{6} \mathrm{D}_{6}\right)$ of the product of 1,2-hydroboration of 3methoxypyridine catalyzed by 1 . Assignment: $\mathrm{P} 1$ = predominant product of 3 -methoxy-1,2dihydropyridine. 


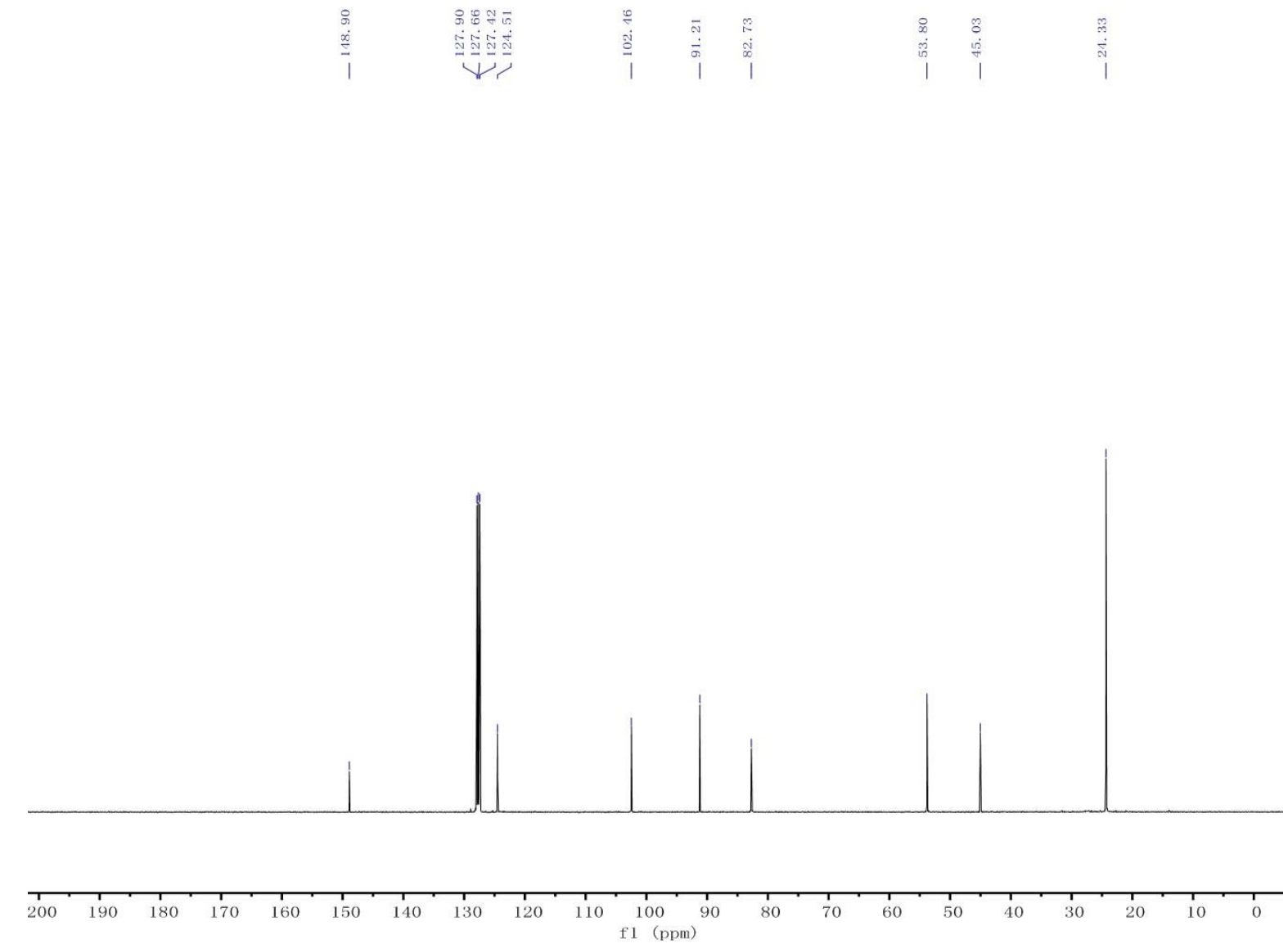

Figure S24. ${ }^{13} \mathrm{C}-\mathrm{NMR}$ spectrum $\left(125 \mathrm{MHz}, \mathrm{C}_{6} \mathrm{D}_{6}\right)$ of the product of 1,2-hydroboration of 3methoxypyridine catalyzed by 1 . 


\section{1-(4,4,5,5-Tetramethyl-1,3,2-dioxaborolan-2-yl)-3-fluoro-1,2-dihydropyridine (2g):}

Colorless liquid (202 mg, 90\%):

${ }^{1} \mathrm{H}$ NMR (500 MHz, $\left.\mathrm{C}_{6} \mathrm{D}_{6}\right): 6.38(\mathrm{dd}, J=5.0 \mathrm{~Hz}, 1 \mathrm{H}), 5.32(\mathrm{dd}, J=5.0 \mathrm{~Hz}, J=15.0 \mathrm{~Hz}, 1 \mathrm{H})$, $4.72(\mathrm{~m}, 1 \mathrm{H}), 4.28(\mathrm{~s}, 2 \mathrm{H}), 0.97$ (s, 12H) ppm.

${ }^{13} \mathrm{C}$ NMR (125 MHz, $\left.\mathrm{C}_{6} \mathrm{D}_{6}\right): 152.7,150.0,127.2,127.1,99.7,99.6,98.9,98.8,43.2,42.9,24.2$ ppm.

Anal. Calc. for $\mathrm{C}_{11} \mathrm{H}_{17} \mathrm{BFNO}_{2}$ : C, 58.70; H, 7.61; N, 6.22. Found: C, 58.68; H, 7.54; N, 6.22.

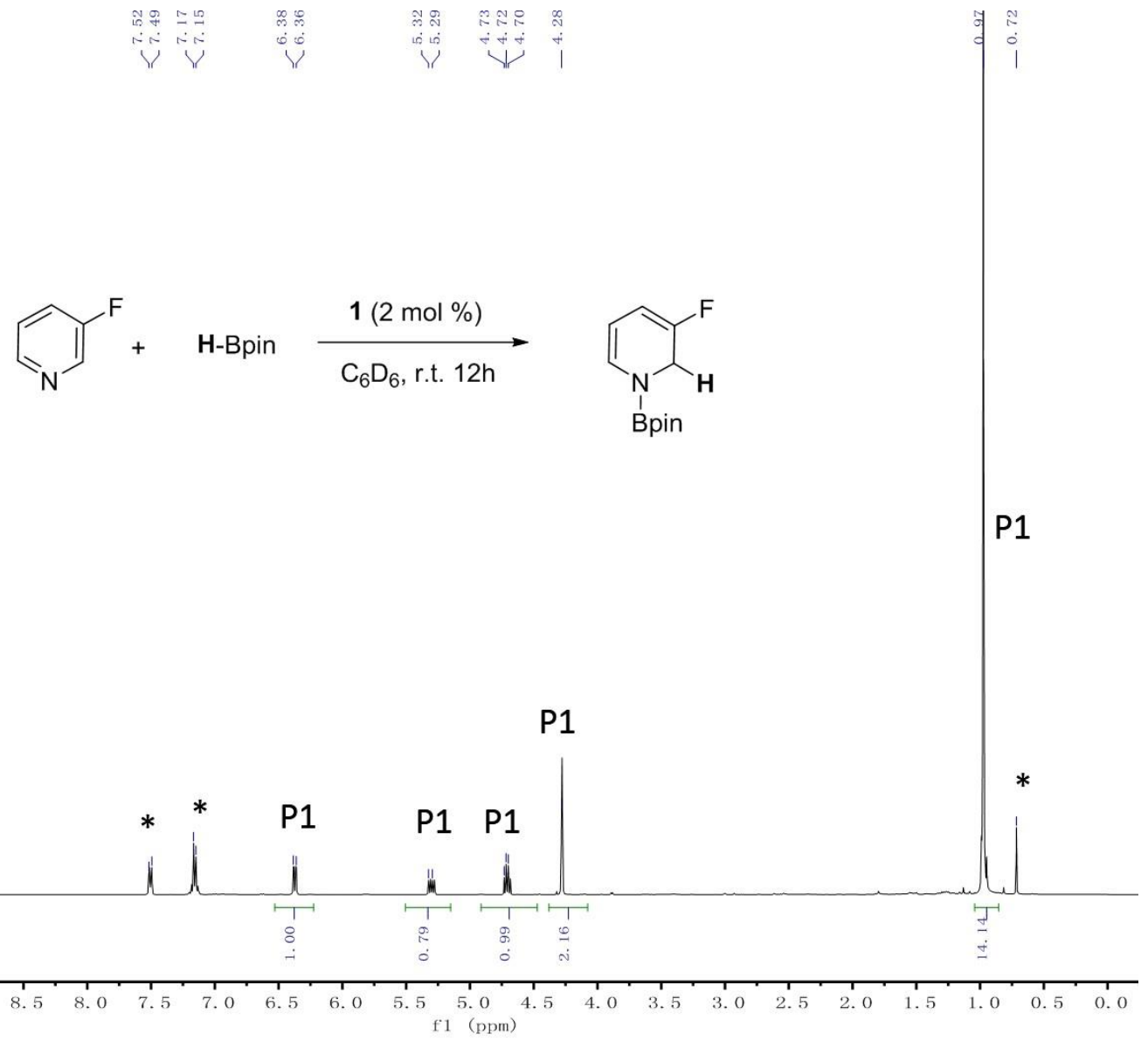

Figure S25. ${ }^{1} \mathrm{H}-\mathrm{NMR}$ spectrum $\left(500 \mathrm{MHz}, \mathrm{C}_{6} \mathrm{D}_{6}\right)$ of the product of 1,2-hydroboration of 3fluoropyridine catalyzed by 1 . Assignment: P1 = predominant product; $*$ internal standard (triphenylmethylsilane). 


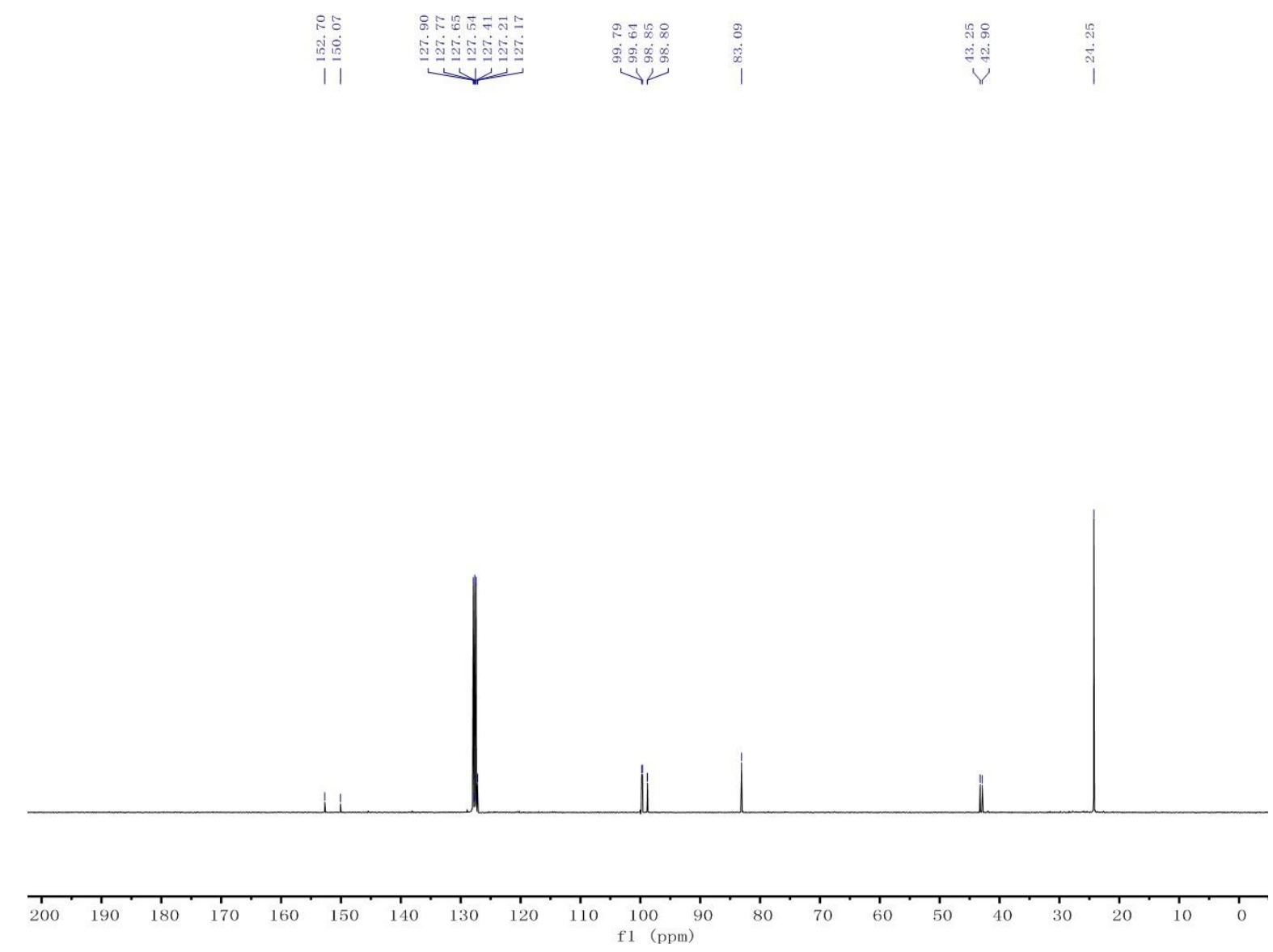

Figure S26. ${ }^{13} \mathrm{C}-\mathrm{NMR}$ spectrum $\left(125 \mathrm{MHz}, \mathrm{C}_{6} \mathrm{D}_{6}\right)$ of the product of 1,2-hydroboration of 3fluoropyridine catalyzed by 1 . 


\section{1-(4,4,5,5-Tetramethyl-1,3,2-dioxaborolan-2-yl)-3-chloro-1,2-dihydropyridine (2h):}

Colorless liquid (219 mg, 91\%):

${ }^{1} \mathrm{H}$ NMR (500 MHz, C $\left.6 \mathrm{D}_{6}\right): 6.26(\mathrm{~d}, J=10.0 \mathrm{~Hz}, 1 \mathrm{H}), 5.54(\mathrm{~d}, J=5.0 \mathrm{~Hz}, 1 \mathrm{H}), 4.52$ (dd, $J=5.0$ $\mathrm{Hz}, 1 \mathrm{H}), 4.07$ (s, 2H), $0.72(\mathrm{~s}, 12 \mathrm{H}) \mathrm{ppm}$.

${ }^{13} \mathrm{C}$ NMR (125 MHz, C6 $\mathrm{D}_{6}$ ): 130.0, 121.0, 119.1, 101.3, 83.1, 48.1, $24.2 \mathrm{ppm}$.

Anal. Calc. for $\mathrm{C}_{11} \mathrm{H}_{17} \mathrm{BCINO}_{2}$ : C, 54.70; H, 7.10; N, 5.80. Found: C, 54.66; H, 6.98; N, 5.78.

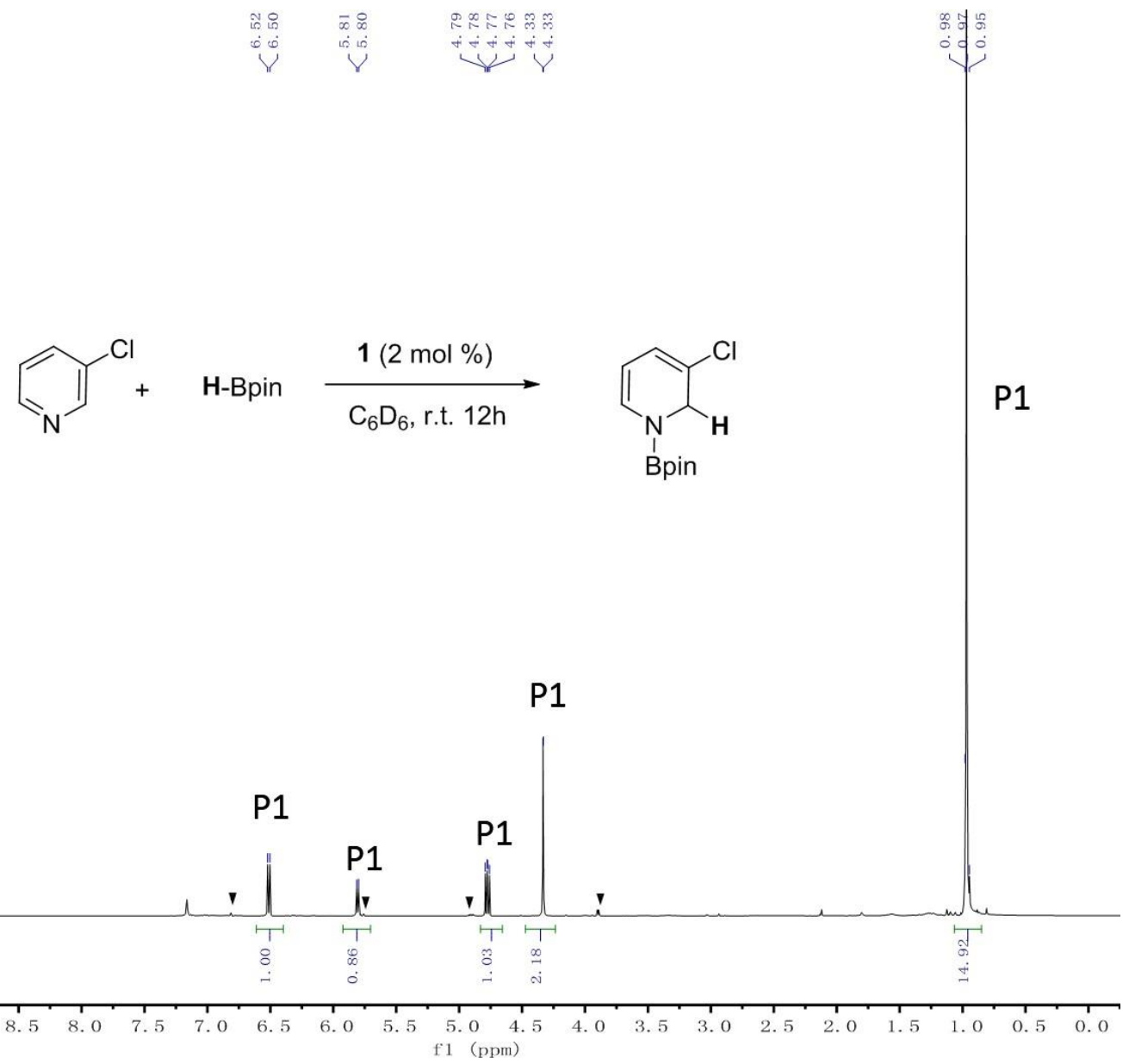

Figure S27. ${ }^{1} \mathrm{H}-\mathrm{NMR}$ spectrum $\left(500 \mathrm{MHz}, \mathrm{C}_{6} \mathrm{D}_{6}\right)$ of the product of 1,2-hydroboration of 3chloropyridine catalyzed by 1 . Assignment: P1 = predominant product of 3-chloro-1,2dihydropyridine; ${ }^{\top}$ 5-chloro-1,2-dihydropyridine. 


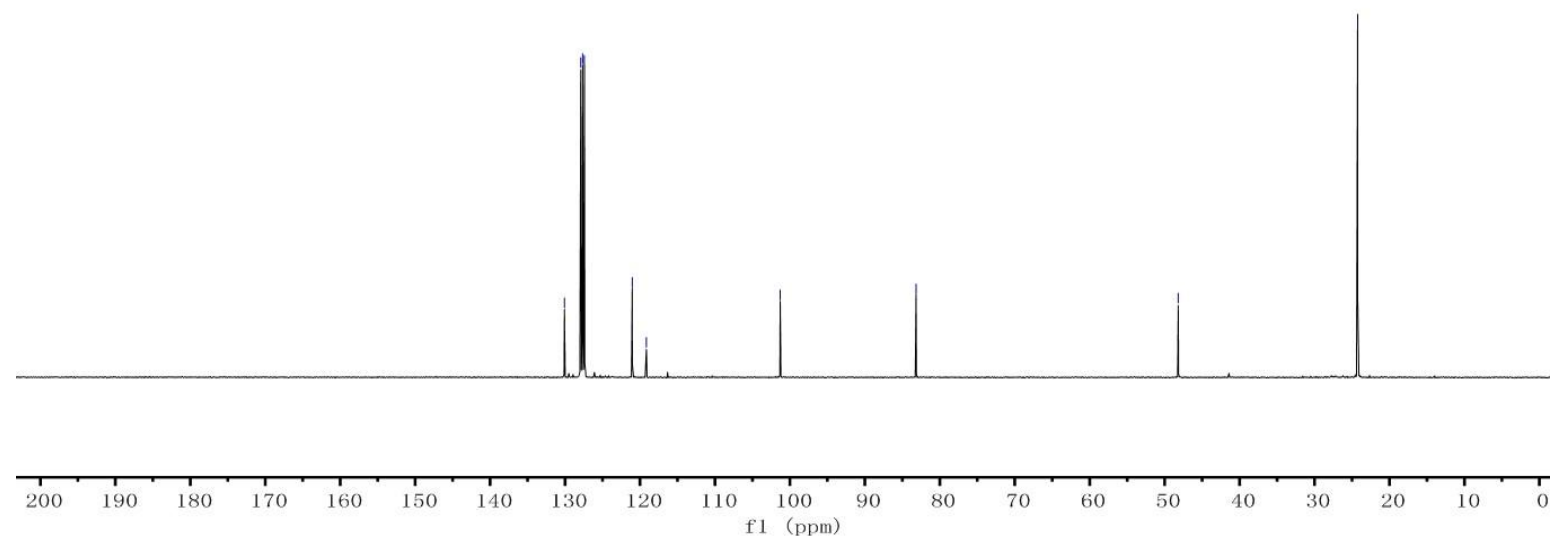

Figure S28. ${ }^{13} \mathrm{C}-\mathrm{NMR}$ spectrum $\left(125 \mathrm{MHz}, \mathrm{C}_{6} \mathrm{D}_{6}\right)$ of the product of 1,2-hydroboration of 3chloropyridine catalyzed by $\mathbf{1}$. 


\section{1-(4,4,5,5-Tetramethyl-1,3,2-dioxaborolan-2-yl)-3-bromo-1,2-dihydropyridine (2i):}

Colorless oil (253 mg, 89\%):

${ }^{1} \mathrm{H}$ NMR (500 MHz, $\left.\mathrm{C}_{6} \mathrm{D}_{6}\right): 6.34(\mathrm{~d}, J=5.0 \mathrm{~Hz}, 1 \mathrm{H}), 5.79(\mathrm{~d}, J=10.0 \mathrm{~Hz} 1 \mathrm{H}), 4.52(\mathrm{dd}, J=10.0$ $\mathrm{Hz}, 1 \mathrm{H}), 4.19$ (s, 2H), 0.73 (s, 12H) ppm.

${ }^{13} \mathrm{C}$ NMR (125 MHz, $\left.\mathrm{C}_{6} \mathrm{D}_{6}\right)$ : 130.5, 125.2, 108.1, 102.0, 83.1, 49.9, $24.2 \mathrm{ppm}$.

Anal. Calc. for $\mathrm{C}_{11} \mathrm{H}_{17} \mathrm{BBrNO}_{2}$ : C, 46.20; H, 5.99; N, 4.90. Found: C, 46.15; H, 5.75; N, 4.87.

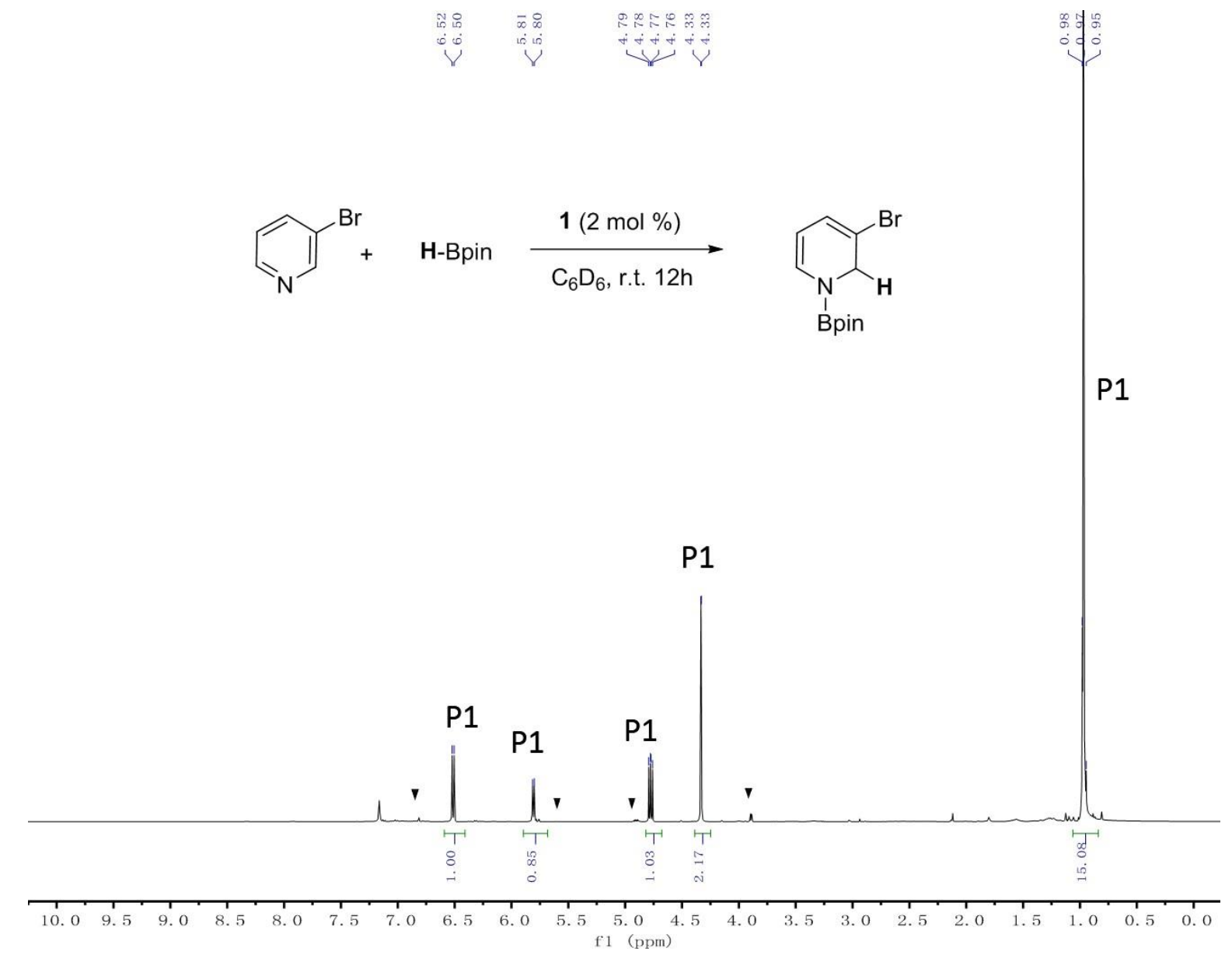

Figure S29. ${ }^{1} \mathrm{H}-\mathrm{NMR}$ spectrum $\left(500 \mathrm{MHz}, \mathrm{C}_{6} \mathrm{D}_{6}\right)$ of the product of 1,2-hydroboration of 3bromopyridine catalyzed by 1 . Assignment: P1 = predominant product of 3-bromo-1,2dihydropyridine; ${ }^{\top}$ 5-bromo-1,2-dihydropyridine. 


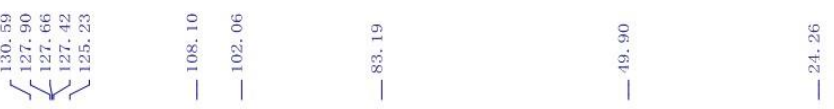
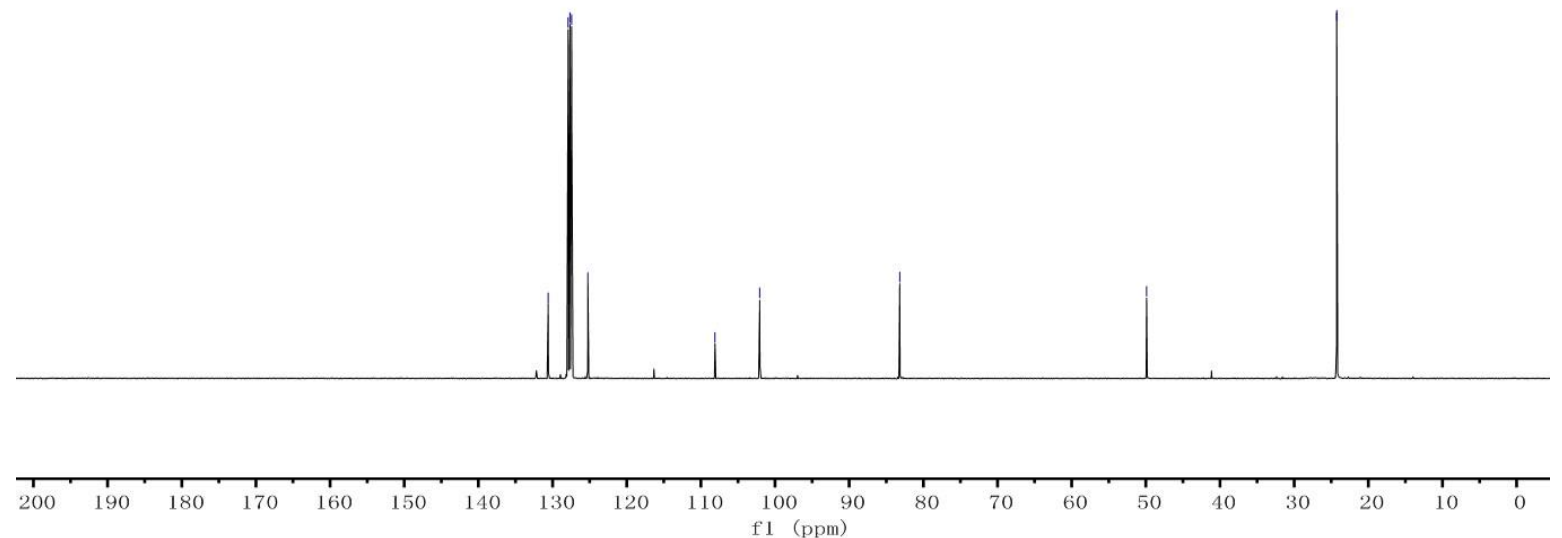

Figure S30. ${ }^{13} \mathrm{C}-\mathrm{NMR}$ spectrum $\left(125 \mathrm{MHz}, \mathrm{C}_{6} \mathrm{D}_{6}\right)$ of the product of 1,2-hydroboration of 3bromopyridine catalyzed by $\mathbf{1}$. 


\section{1-(4,4,5,5-Tetramethyl-1,3,2-dioxaborolan-2-yl)-3-iodo-1,2-dihydropyridine (2j):}

Colorless oil (259 mg, 78\%):

${ }^{1} \mathrm{H}$ NMR $\left(500 \mathrm{MHz}, \mathrm{C}_{6} \mathrm{D}_{6}\right): 6.65(\mathrm{~d}, J=5.0 \mathrm{~Hz}, 1 \mathrm{H}), 6.28(\mathrm{~d}, J=5.0 \mathrm{~Hz} 1 \mathrm{H}), 4.68(\mathrm{dd}, J=5.0$ $\mathrm{Hz}, 1 \mathrm{H}), 4.43$ (s, 2H), 1.00 (s, 12H) ppm.

${ }^{13} \mathrm{C}$ NMR (125 MHz, $\left.\mathrm{C}_{6} \mathrm{D}_{6}\right): 133.5,131.4,103.5,83.1,82.7,53.1,24.2 \mathrm{ppm}$.

Anal. Calc. for $\mathrm{C}_{11} \mathrm{H}_{17} \mathrm{BINO}_{2}$ : C, 39.68; H, 5.15; N, 4.21. Found: C, 69.60; H, 5.01; N, 4.23.
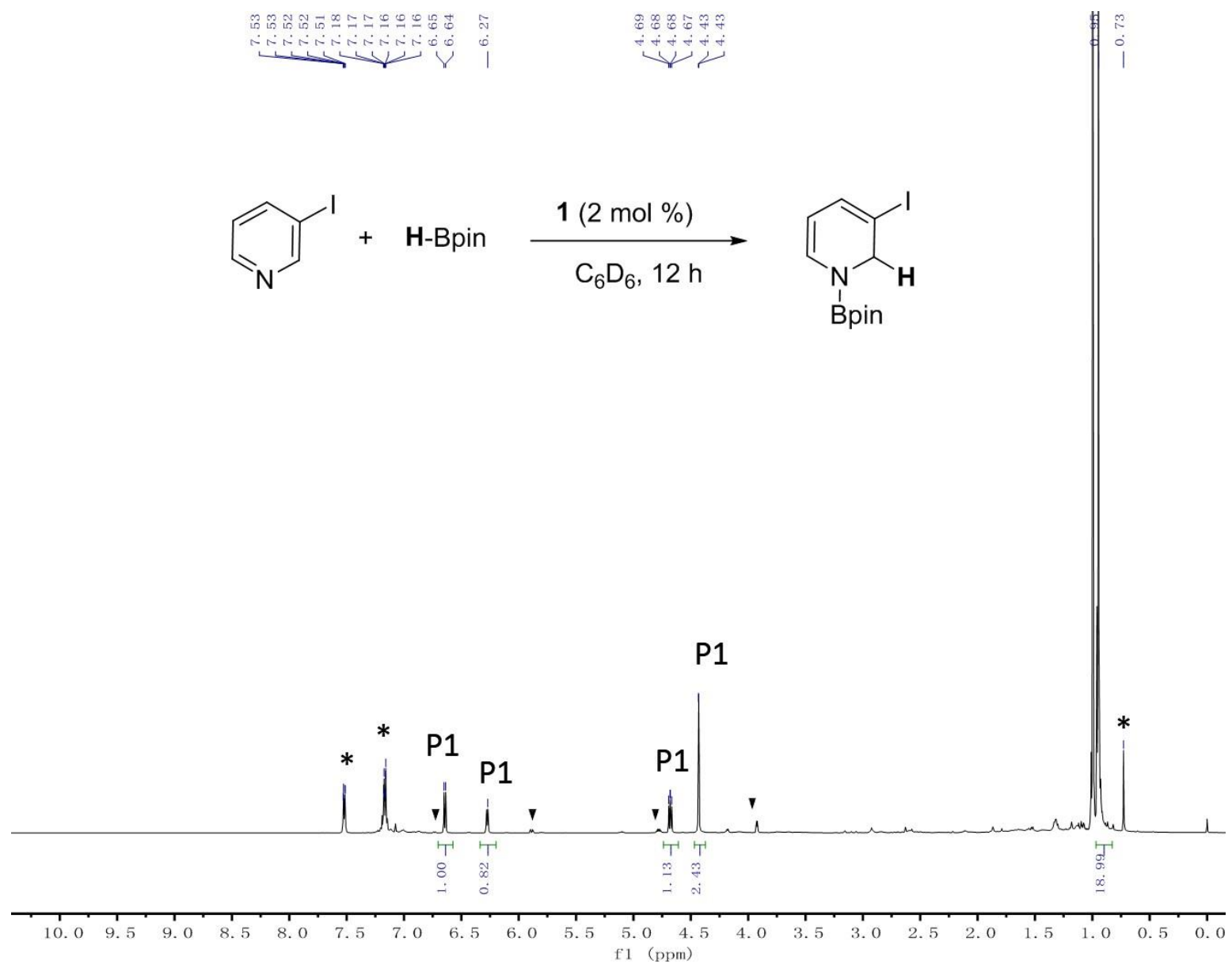

Figure S31. ${ }^{1} \mathrm{H}-\mathrm{NMR}$ spectrum $\left(500 \mathrm{MHz}, \mathrm{C}_{6} \mathrm{D}_{6}\right)$ of the product of 1,2-hydroboration of 3iodopyridine catalyzed by 1 . Assignment: P1 = predominant product of 3-iodo-1,2dihydropyridine; * internal standard (triphenylmethylsilane); 5-iodo-1,2-dihydropyridine. 


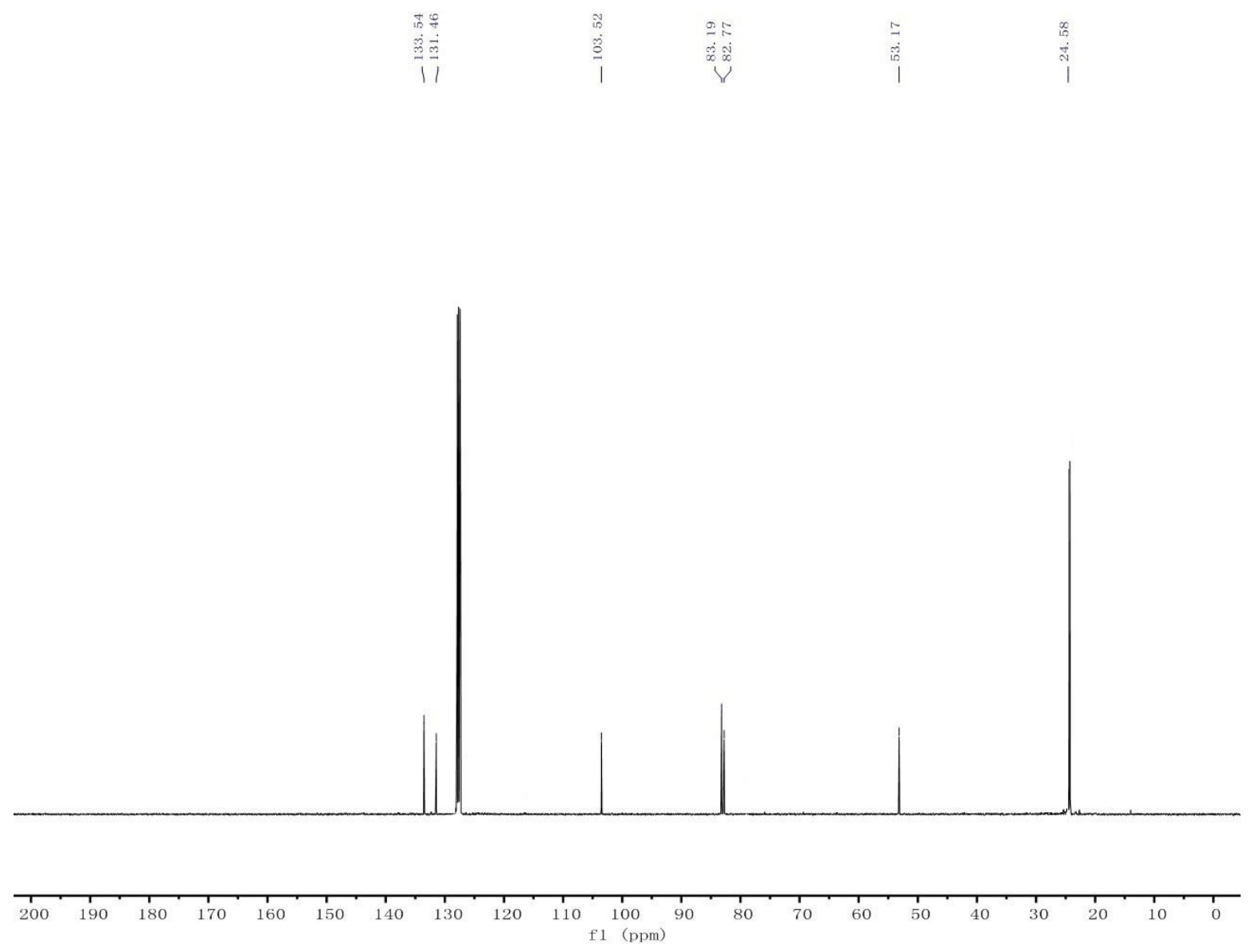

Figure S32. ${ }^{13} \mathrm{C}-\mathrm{NMR}$ spectrum $\left(125 \mathrm{MHz}, \mathrm{C}_{6} \mathrm{D}_{6}\right)$ of the product of 1,2-hydroboration of 3iodopyridine catalyzed by $\mathbf{1}$. 


\section{1-(4,4,5,5-tetramethyl-1,3,2-dioxaborolan-2-yl)-1,2-dihydroquinoline (2k):}

White solid (239 mg, 93\%):

${ }^{1} \mathrm{H}$ NMR $\left(500 \mathrm{MHz}, \mathrm{C}_{6} \mathrm{D}_{6}\right): 7.83(\mathrm{~d}, J=10.0 \mathrm{~Hz}, 1 \mathrm{H}), 7.16(\mathrm{t}, J=10.0 \mathrm{~Hz}, 1 \mathrm{H}), 6.85-6.81(\mathrm{~m}$, $2 \mathrm{H}), 6.27(\mathrm{~d}, J=10.0 \mathrm{~Hz}, 1 \mathrm{H}), 5.58(\mathrm{td}, J=10.0 \mathrm{~Hz}, 1 \mathrm{H}), 4.17(\mathrm{~s}, 2 \mathrm{H}), 1.05(\mathrm{~s}, 12 \mathrm{H}) \mathrm{ppm}$.

${ }^{13} \mathrm{C}$ NMR $\left(125 \mathrm{MHz}, \mathrm{C}_{6} \mathrm{D}_{6}\right)$ : 141.9, 127.8,127.5, 126.7, 126.5, 124.2, 121.5, 120.8, 82.4, 43.2, $24.3 \mathrm{ppm}$.

Anal. Calc. for $\mathrm{C}_{15} \mathrm{H}_{20} \mathrm{BNO}_{2}$ : C, 70.06; H, 7.84; N, 5.45. Found: C, 70.10; H, 7.56; N, 5.43.

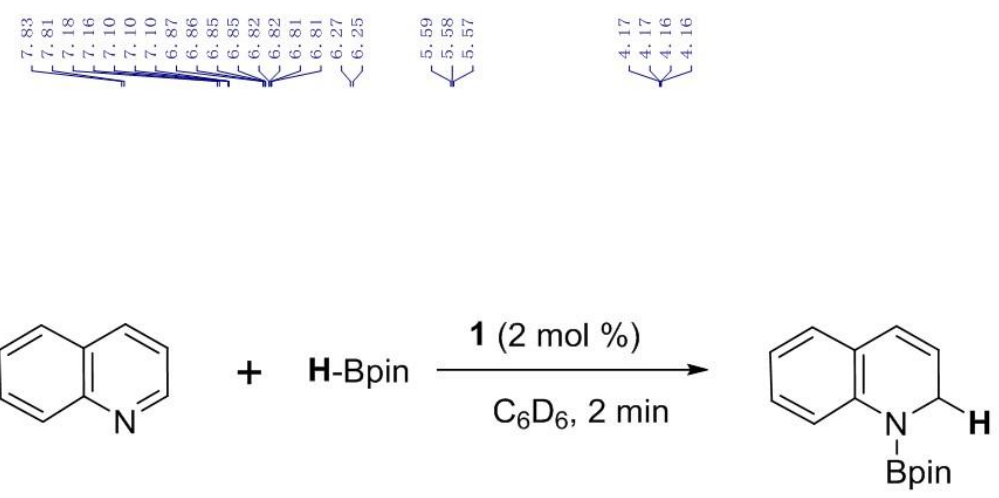

P1

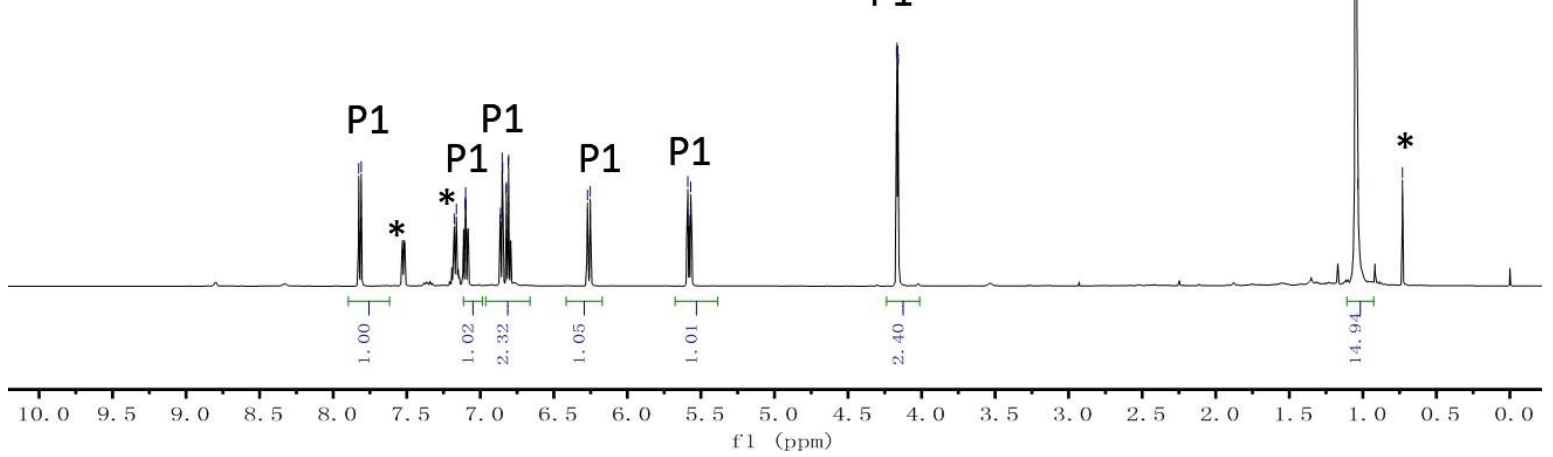

Figure S33. ${ }^{1} \mathrm{H}-\mathrm{NMR}$ spectrum $\left(500 \mathrm{MHz}, \mathrm{C}_{6} \mathrm{D}_{6}\right)$ of the product of 1,2-hydroboration of quinoline catalyzed by 1. Assignment: P1 = predominant product; * internal standard (triphenylmethylsilane). 

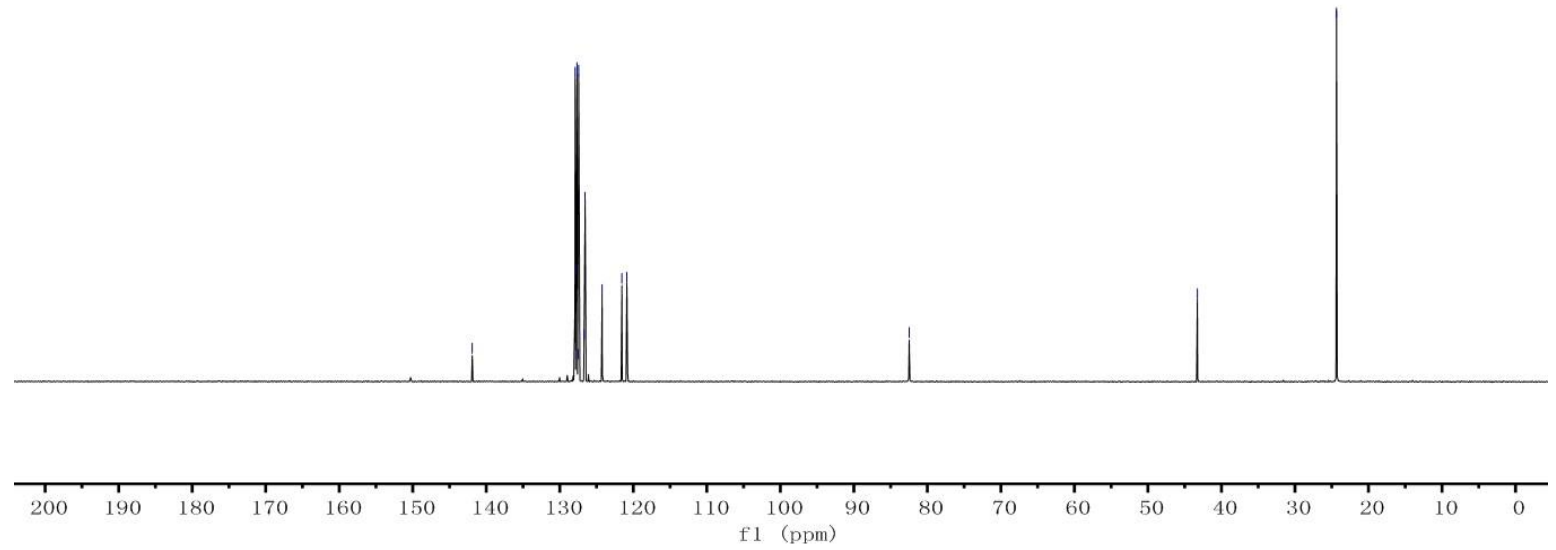

Figure S34. ${ }^{13} \mathrm{C}-\mathrm{NMR}$ spectrum $\left(125 \mathrm{MHz}, \mathrm{C}_{6} \mathrm{D}_{6}\right)$ of the product of 1,2-hydroboration of quinoline catalyzed by $\mathbf{1}$. 


\section{2-(4,4,5,5-tetramethyl-1,3,2-dioxaborolan-2-yl)-1,2-dihydroisoquinoline (2l):}

Colorless oil (233 mg, 91\%):

${ }^{1} \mathrm{H}$ NMR $\left(500 \mathrm{MHz}, \mathrm{C}_{6} \mathrm{D}_{6}\right): 6.98(\mathrm{~d}, J=10.0 \mathrm{~Hz}, 1 \mathrm{H}), 6.88(\mathrm{td}, J=5.0 \mathrm{~Hz}, J=10.0 \mathrm{~Hz}, 1 \mathrm{H})$, $6.82(\mathrm{td}, J=5.0 \mathrm{~Hz}, J=10.0 \mathrm{~Hz}, 1 \mathrm{H}), 6.79(\mathrm{t}, J=10.0 \mathrm{~Hz}, 1 \mathrm{H}), 6.72(\mathrm{~d}, J=10.0 \mathrm{~Hz}, 1 \mathrm{H}), 5.62$ $(\mathrm{d}, J=10.0 \mathrm{~Hz}, 1 \mathrm{H}), 4.62(\mathrm{~s}, 2 \mathrm{H}), 1.01(\mathrm{~s}, 12 \mathrm{H}) \mathrm{ppm}$.

${ }^{13} \mathrm{C}$ NMR $\left(125 \mathrm{MHz}, \mathrm{C}_{6} \mathrm{D}_{6}\right): 133.2,132.7,128.6,127.2,125.7,125.0,123.3,105.8,82.9,45.8$, $24.3 \mathrm{ppm}$.

Anal. Calc. for $\mathrm{C}_{15} \mathrm{H}_{20} \mathrm{BNO}_{2}$ : C, 70.06; H, 7.84; N, 5.45. Found: C, 70.05; H, 7.60; N, 5.43.

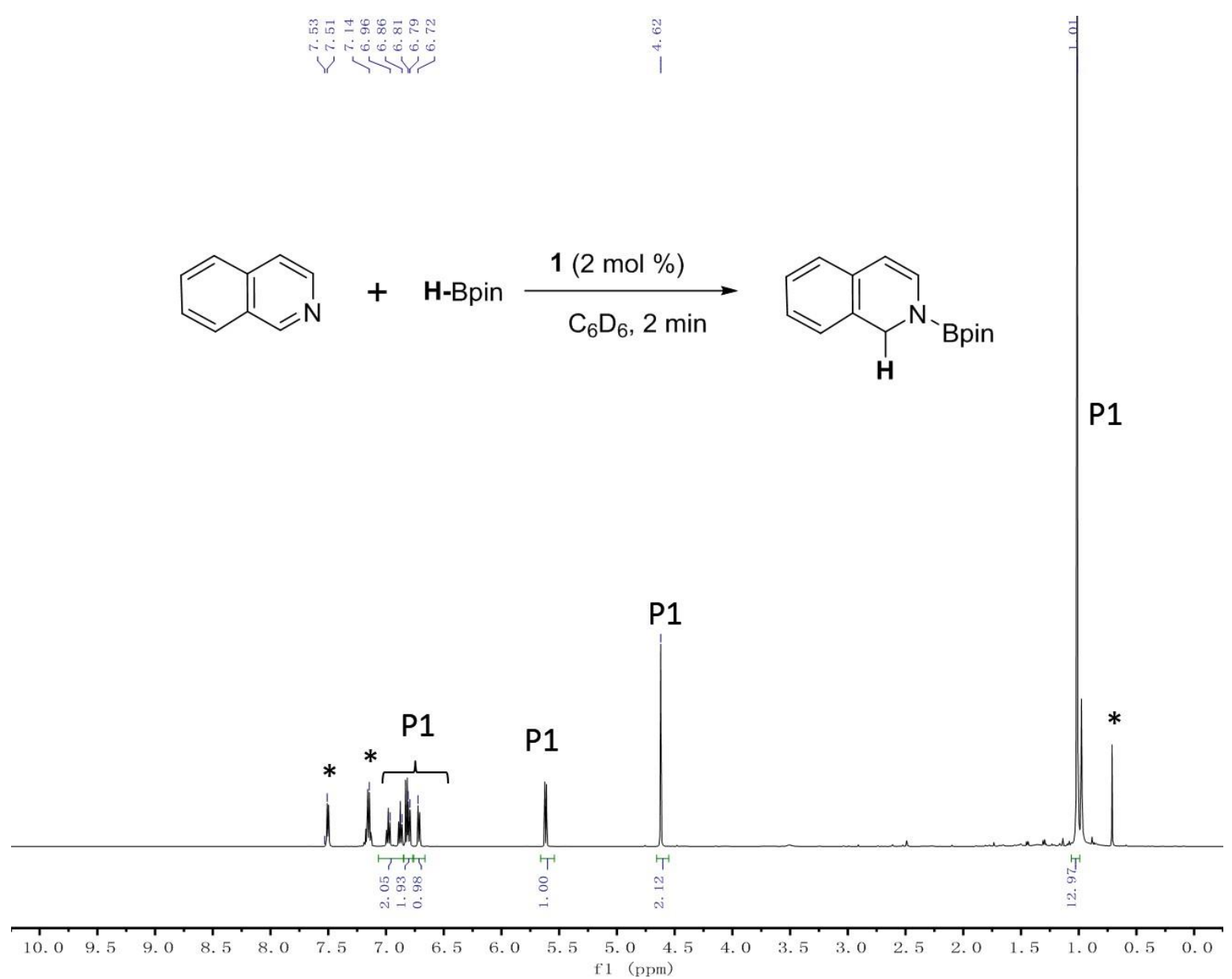

Figure S35. ${ }^{1} \mathrm{H}-\mathrm{NMR}$ spectrum $\left(500 \mathrm{MHz}, \mathrm{C}_{6} \mathrm{D}_{6}\right)$ of the product of 1,2-hydroboration of isoquinoline catalyzed by 1 . Assignment: $\mathrm{P} 1=$ predominant product; $*$ internal standard (triphenylmethylsilane). 


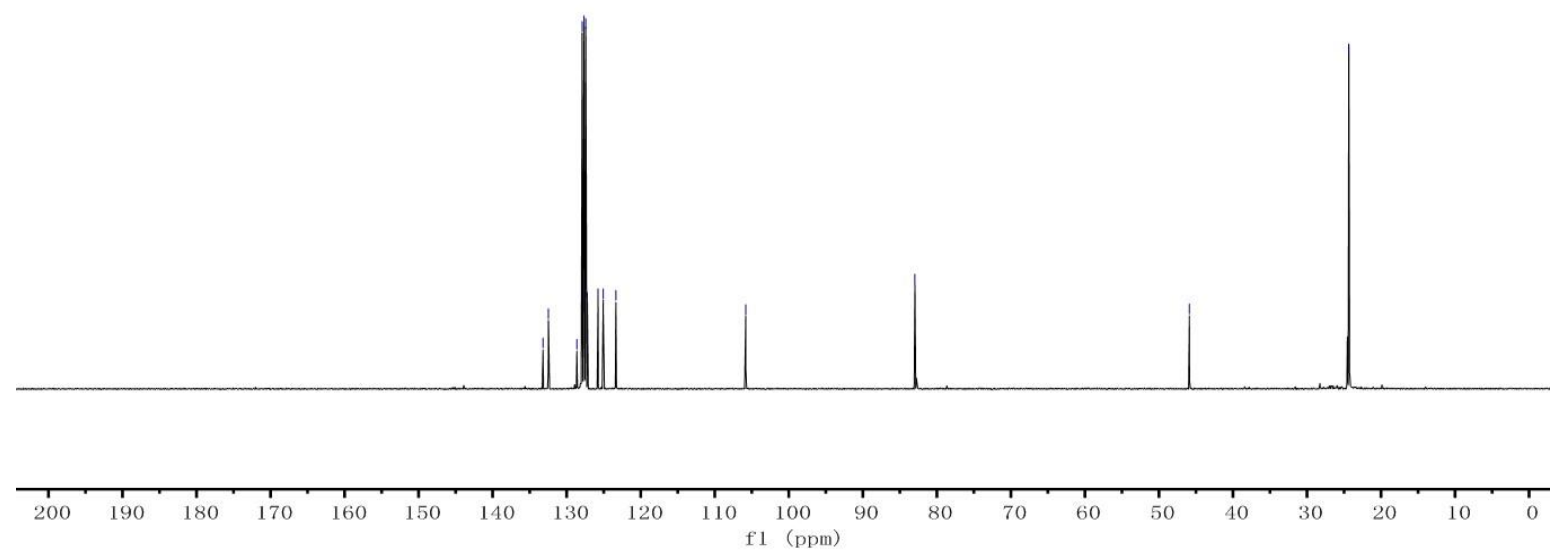

Figure S36. ${ }^{13} \mathrm{C}-\mathrm{NMR}$ spectrum $\left(125 \mathrm{MHz}, \mathrm{C}_{6} \mathrm{D}_{6}\right)$ of the product of 1,2-hydroboration of isoquinoline catalyzed by $\mathbf{1}$. 


\section{3-Me-1-(4,4,5,5-tetramethyl-1,3,2-dioxaborolan-2-yl)-1,2-dihydroquinoline (2m):}

White solid (244 mg, 90\%):

${ }^{1} \mathrm{H}$ NMR $\left(500 \mathrm{MHz}, \mathrm{C}_{6} \mathrm{D}_{6}\right): 7.85(\mathrm{~d}, J=10.0 \mathrm{~Hz}, 1 \mathrm{H}), 7.09(\mathrm{td}, J=10.0 \mathrm{~Hz}, 1 \mathrm{H}), 6.86-6.83(\mathrm{~m}$, 2H), 6.00 (s, 1H), 4.08 (s, 2H), 1.48 (s, 3H), 1.04 (s, 12H) ppm.

${ }^{13} \mathrm{C}$ NMR $\left(125 \mathrm{MHz}, \mathrm{C}_{6} \mathrm{D}_{6}\right): 140.3,133.6,127.3,126.9,125.7,121.6,121.5,120.3,82.4,47.9$, 24.3, $20.1 \mathrm{ppm}$.

Anal. Calc. for $\mathrm{C}_{16} \mathrm{H}_{22} \mathrm{BNO}_{2}$ : C, 70.87; H, 8.18; N, 5.17. Found: C, 70.85; H, 8.03; N, 5.15.

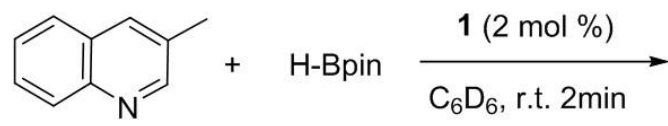<smiles>[B]N1CC(C)=Cc2ccccc21</smiles>

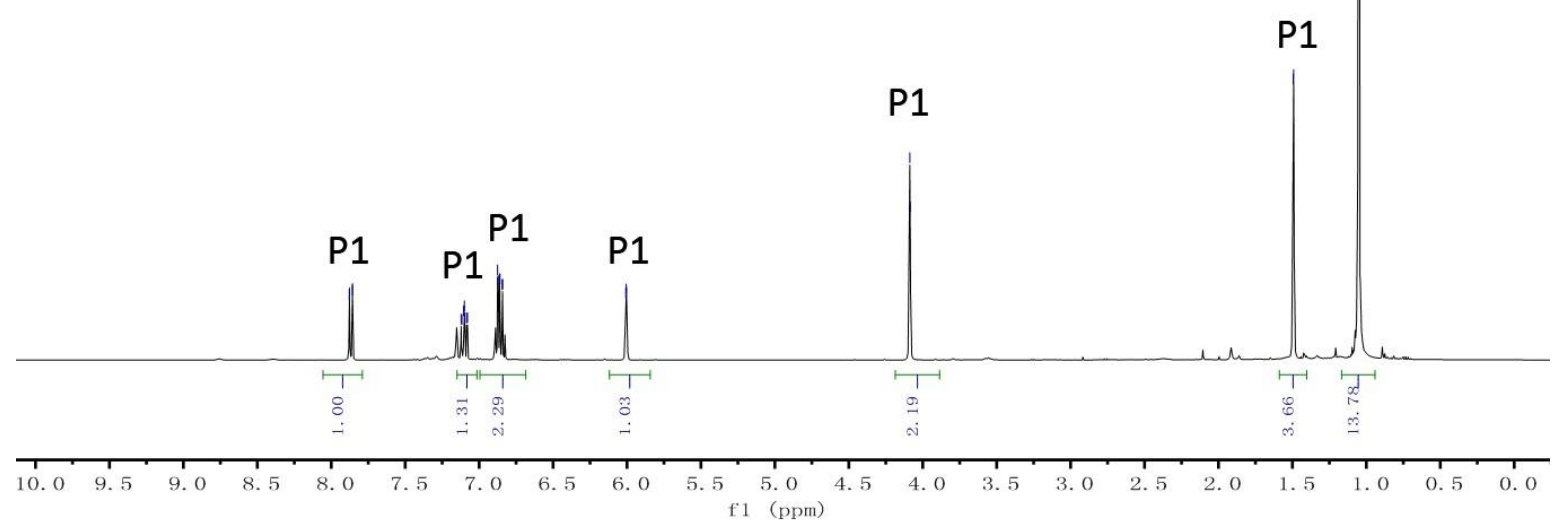

Figure S37. ${ }^{1} \mathrm{H}-\mathrm{NMR}$ spectrum $\left(500 \mathrm{MHz}, \mathrm{C}_{6} \mathrm{D}_{6}\right)$ of the product of hydroboration of 3-Mequinoline catalyzed by $\mathbf{1}$. Assignment: $\mathrm{P} 1=$ predominant product . 

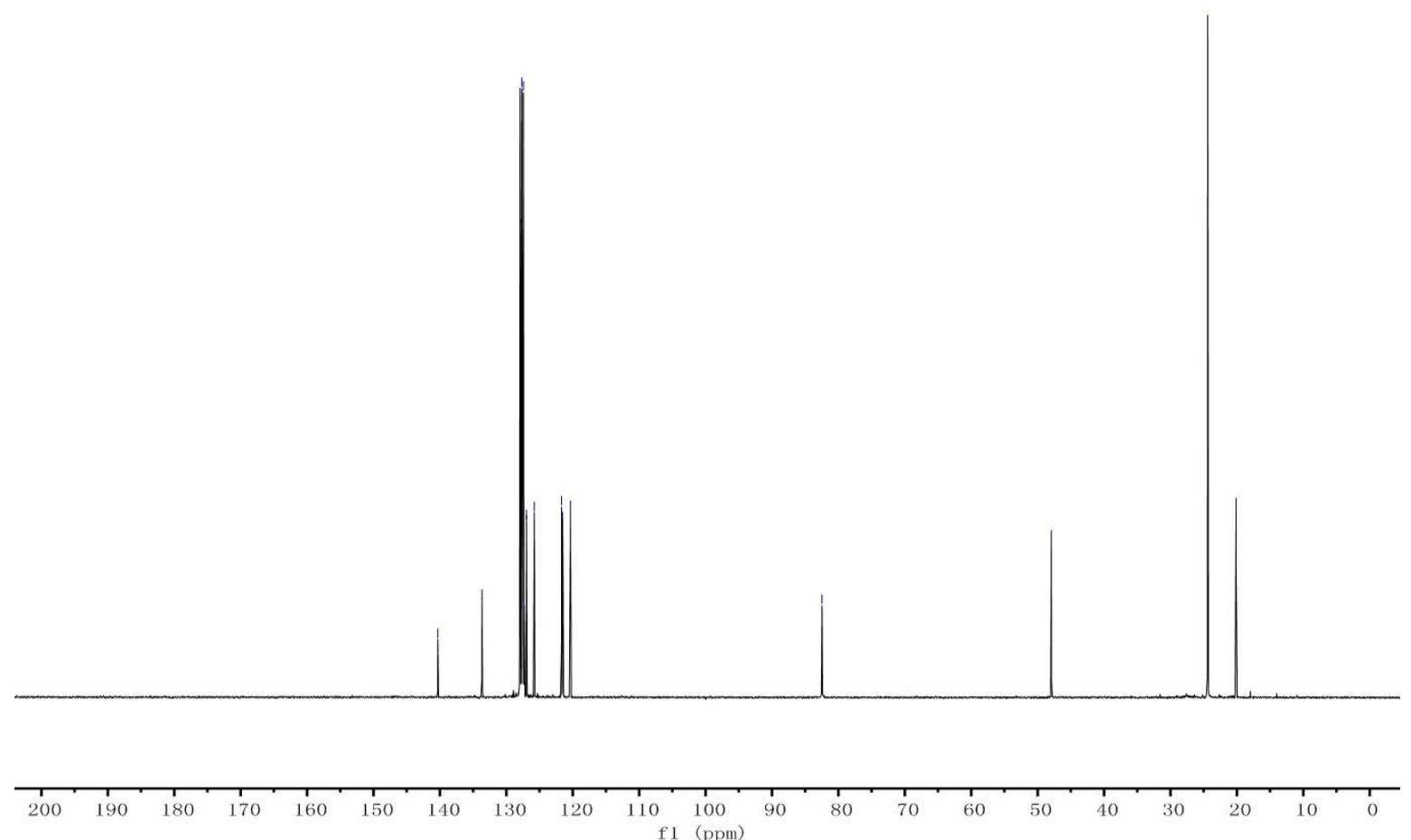

Figure S38. ${ }^{13} \mathrm{C}$-NMR spectrum $\left(125 \mathrm{MHz}, \mathrm{C}_{6} \mathrm{D}_{6}\right)$ of the product of 1,2-hydroboration of 3-Mequinoline catalyzed by $\mathbf{1}$. 


\section{1-Me-2-(4,4,5,5-tetramethyl-1,3,2-dioxaborolan-2-yl)-1,2-dihydroisoquinoline (2n):}

White solid (224 mg, 83\%):

${ }^{1} \mathrm{H}$ NMR $\left(500 \mathrm{MHz}, \mathrm{C}_{6} \mathrm{D}_{6}\right): 7.00(\mathrm{td}, J=10.0 \mathrm{~Hz}, 1 \mathrm{H}), 6.92(\mathrm{td}, J=10.0 \mathrm{~Hz}, 1 \mathrm{H}), 6.86(\mathrm{~d}, J=$ $5.0 \mathrm{~Hz}, 1 \mathrm{H}), 6.75(\mathrm{~d}, J=10.0 \mathrm{~Hz}, 2 \mathrm{H}), 5.92(\mathrm{~d}, J=5.0 \mathrm{~Hz}, 1 \mathrm{H}), 5.02(\mathrm{q}, J=5.0 \mathrm{~Hz}, 1 \mathrm{H}), 1.35$ (d, $J=10.0 \mathrm{~Hz}, 3 \mathrm{H}), 1.03(\mathrm{~s}, 12 \mathrm{H}) \mathrm{ppm}$.

${ }^{13} \mathrm{C}$ NMR (125 MHz, $\left.\mathrm{C}_{6} \mathrm{D}_{6}\right): 133.7,131.5,129.9,127.0,125.7,125.3,123.7,104.1,82.9,82.7$, $51.0,25.5 \mathrm{ppm}$.

Anal. Calc. for $\mathrm{C}_{16} \mathrm{H}_{22} \mathrm{BNO}_{2}$ : C, 70.87; H, 8.18; N, 5.17. Found: C, 70.87; H, 8.06; N, 5.14.

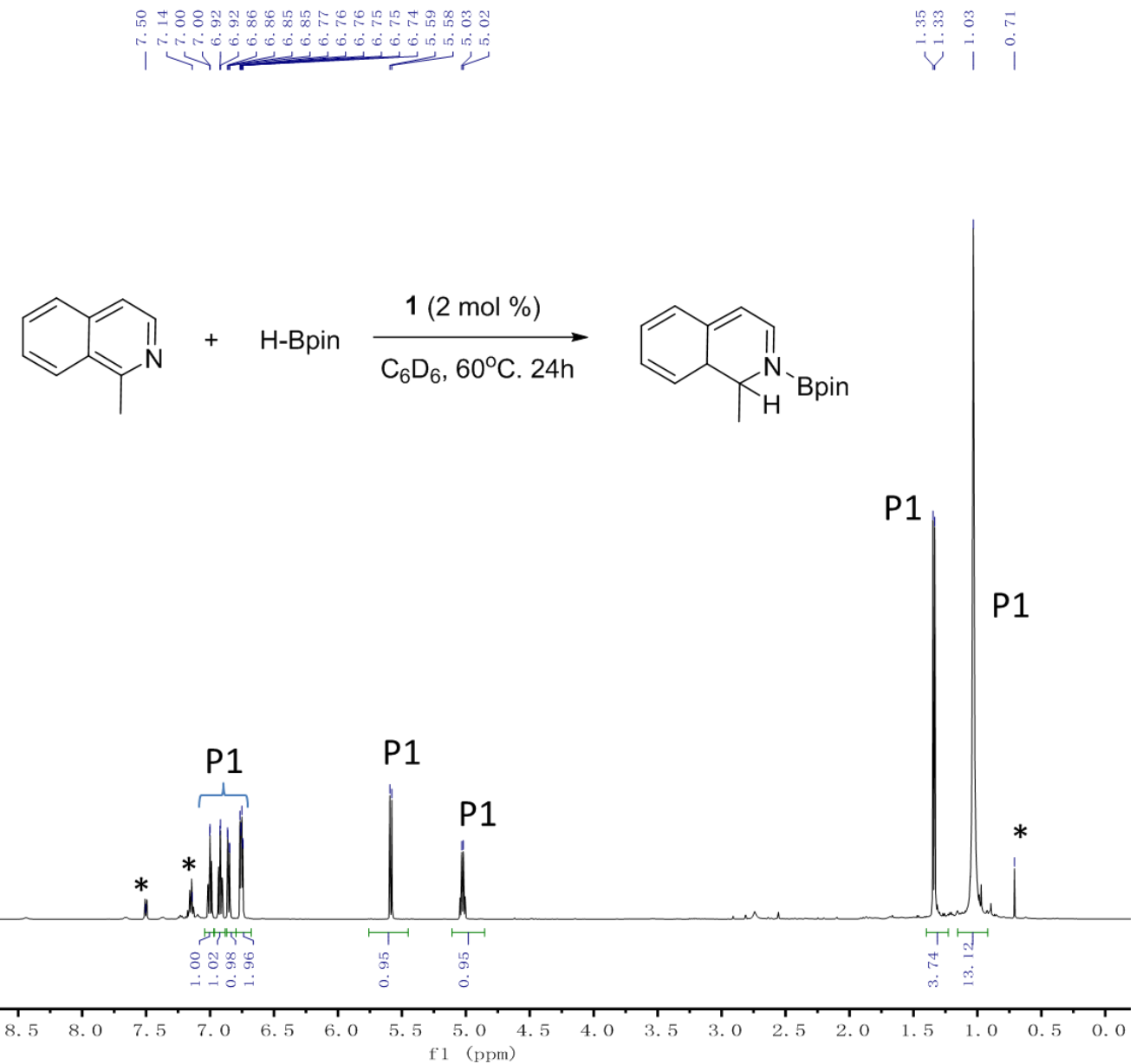

Figure S39. ${ }^{1} \mathrm{H}-\mathrm{NMR}$ spectrum $\left(500 \mathrm{MHz}, \mathrm{C}_{6} \mathrm{D}_{6}\right)$ of the product of hydroboration of 1-Meisoquinoline catalyzed by 1. Assignment: P1 = predominant product; * internal standard (triphenylmethylsilane). 

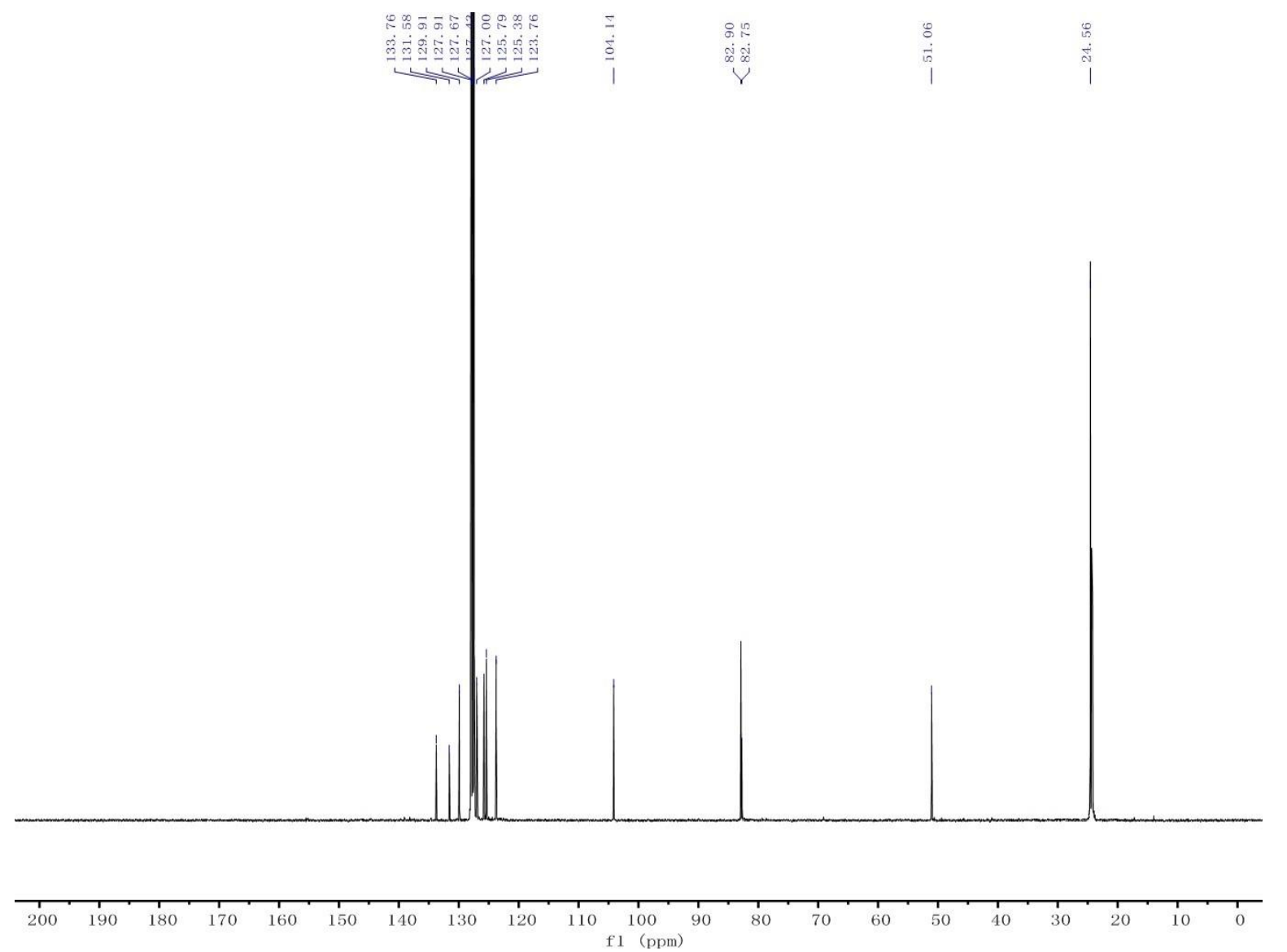

Figure S40. ${ }^{13} \mathrm{C}-\mathrm{NMR}$ spectrum $\left(125 \mathrm{MHz}, \mathrm{C}_{6} \mathrm{D}_{6}\right)$ of the product of 1,2-hydroboration of 1-Meisoquinoline catalyzed by $\mathbf{1}$. 


\section{3-Me-2-(4,4,5,5-tetramethyl-1,3,2-dioxaborolan-2-yl)-1,2-dihydroisoquinoline (2o):}

Colorless solid (249 mg, 92\%):

${ }^{1} \mathrm{H}$ NMR (500 MHz, $\left.\mathrm{C}_{6} \mathrm{D}_{6}\right): 7.02(\mathrm{td}, J=5.0 \mathrm{~Hz}, 1 \mathrm{H}), 6.92(\mathrm{~d}, J=5.0 \mathrm{~Hz}, 1 \mathrm{H}), 6.88(\mathrm{~d}, J=10.0$ $\mathrm{Hz}, 2 \mathrm{H}), 5.72$ (s, 1H), 4.62 (s, 2H), 2.25 (s, 3H), 0.99 (s, 12H) ppm.

${ }^{13} \mathrm{C}$ NMR (125 MHz, $\left.\mathrm{C}_{6} \mathrm{D}_{6}\right)$ : 142.6, 134.3, 129.5, 127.1, 125.1, 124.3, 122.4, 108.4, 82.3, 48.1, 24.2, $21.5 \mathrm{ppm}$.

Anal. Calc. for $\mathrm{C}_{16} \mathrm{H}_{22} \mathrm{BNO}_{2}$ : C, 70.87; H, 8.18; N, 5.17. Found: C, 70.91; H, 8.07; N, 5.17.

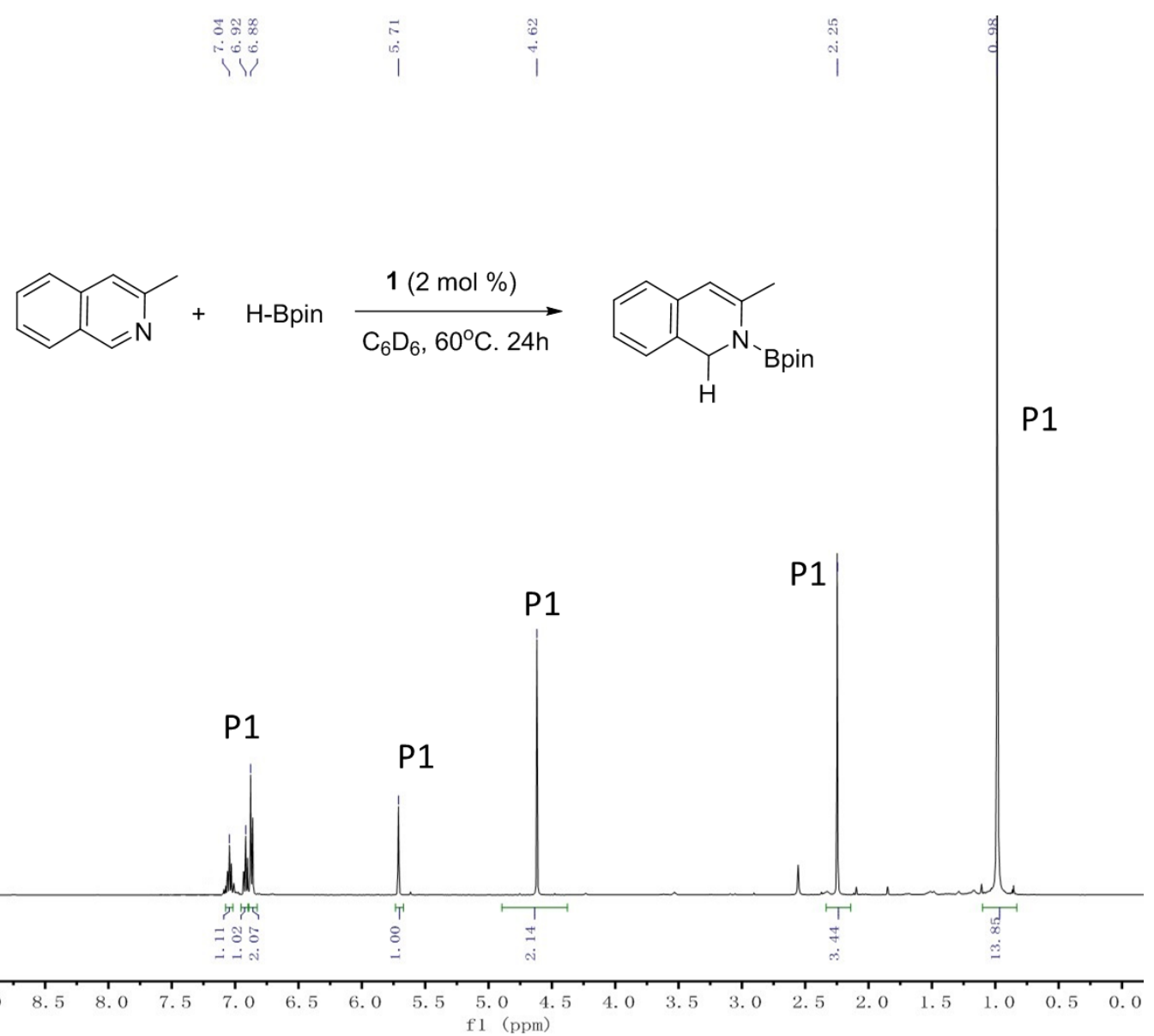

Figure S41. ${ }^{1} \mathrm{H}-\mathrm{NMR}$ spectrum $\left(500 \mathrm{MHz}, \mathrm{C}_{6} \mathrm{D}_{6}\right)$ of the product of hydroboration of 3-Meisoquinoline catalyzed by $\mathbf{1}$. Assignment: $\mathrm{P} 1=$ predominant product. 

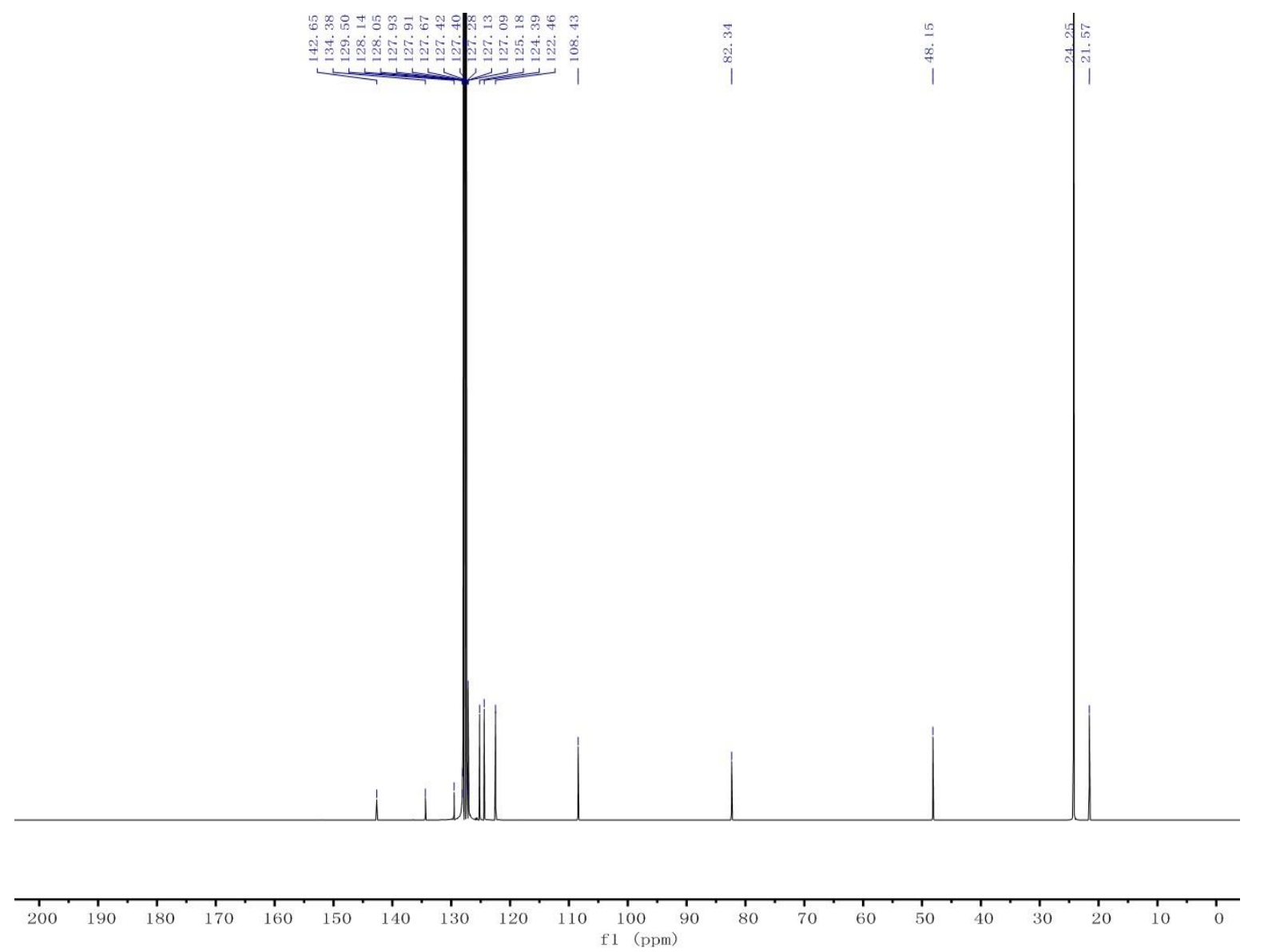

Figure S42. ${ }^{13} \mathrm{C}-\mathrm{NMR}$ spectrum $\left(125 \mathrm{MHz}, \mathrm{C}_{6} \mathrm{D}_{6}\right)$ of the product of 1,2-hydroboration of 3-Meisoquinoline catalyzed by $\mathbf{1}$. 


\section{1,4-bis(4,4,5,5-tetramethyl-1,3,2-dioxaborolan-2-yl)-1,2,3,4-tetrahydropyrazine (2p):}

Colorless oil (262 mg, 78\%):

${ }^{1} \mathrm{H}$ NMR (500 MHz, $\left.\mathrm{C}_{6} \mathrm{D}_{6}\right): 6.13$ (s, 2H), 3.47 (s, 4H), 0.99 (s, 24H) ppm.

${ }^{13} \mathrm{C}$ NMR (125 MHz, $\mathrm{C}_{6} \mathrm{D}_{6}$ ): 110.7, 82.3, 42.1, 24.4 ppm.

Anal. Calc. for $\mathrm{C}_{16} \mathrm{H}_{30} \mathrm{~B}_{2} \mathrm{~N}_{2} \mathrm{O}_{4}$ : C, 57.19; H, 9.00; N, 8.34. Found: C, 57.17; H, 8.87; N, 8.29.

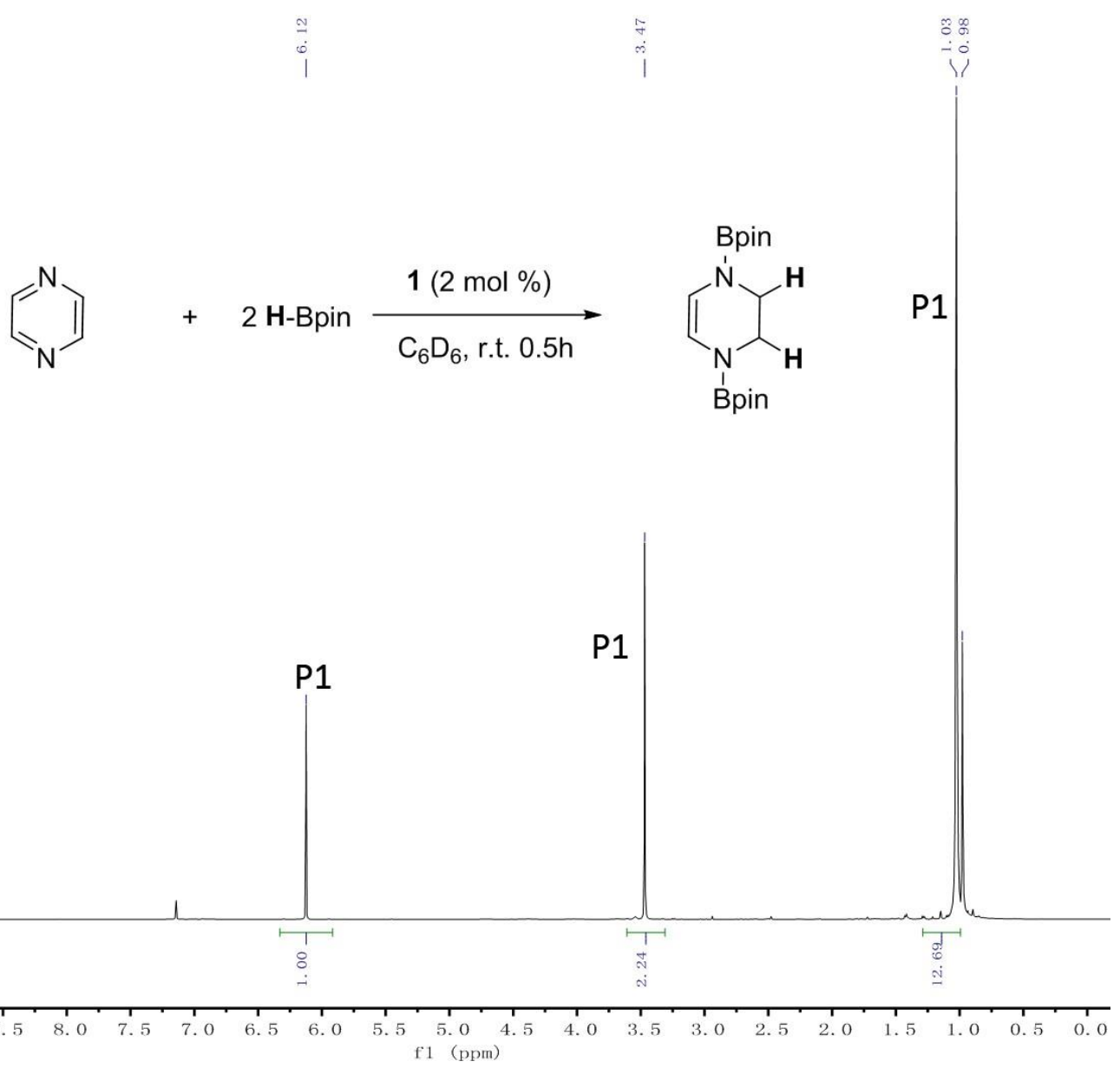

Figure S43. ${ }^{1} \mathrm{H}-\mathrm{NMR}$ spectrum $\left(500 \mathrm{MHz}, \mathrm{C}_{6} \mathrm{D}_{6}\right)$ of the product of 1,2-hydroboration of pyrazine catalyzed by 1 . Assignment: $\mathrm{P} 1=$ predominant product. 

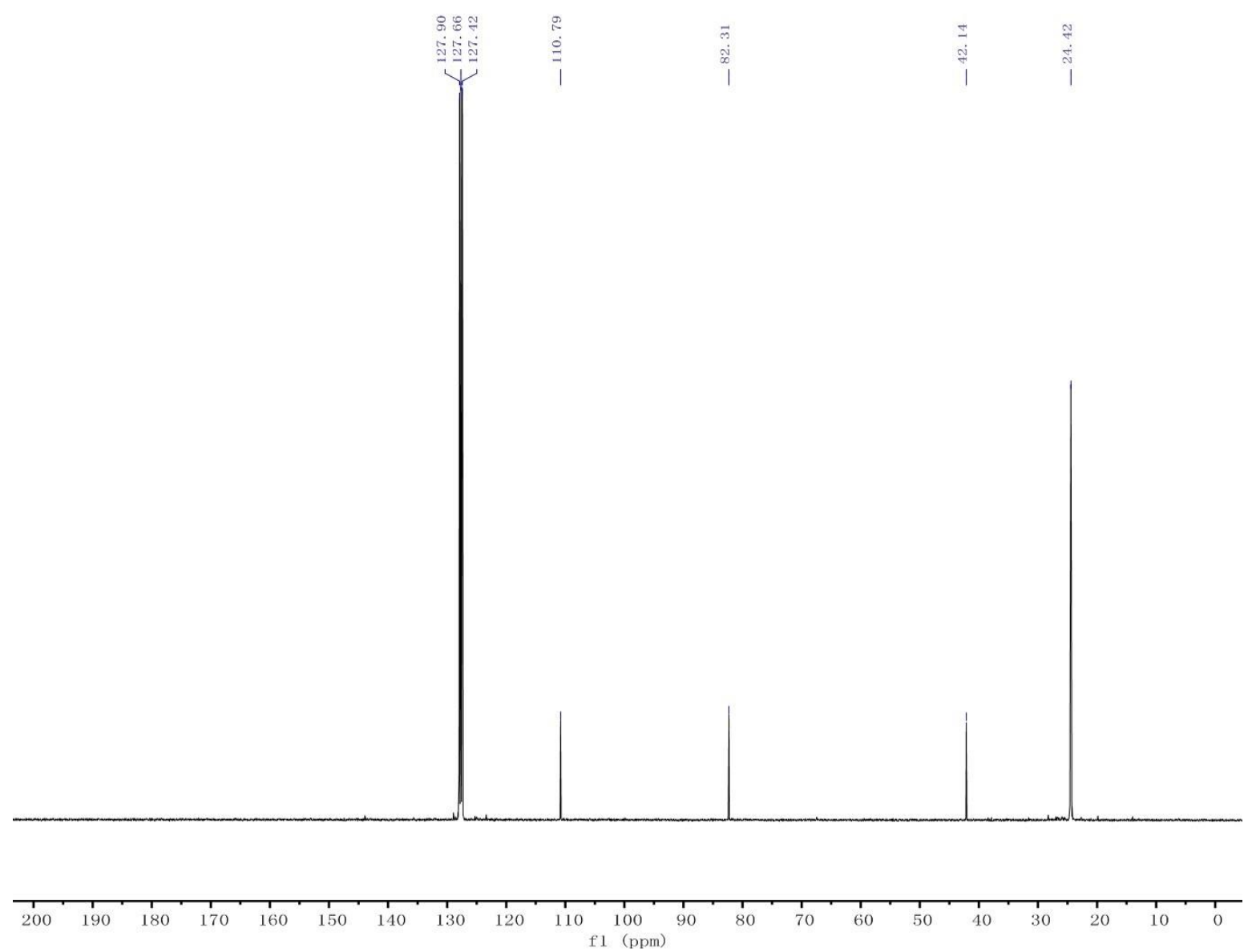

Figure S44. ${ }^{13} \mathrm{C}-\mathrm{NMR}$ spectrum $\left(125 \mathrm{MHz}, \mathrm{C}_{6} \mathrm{D}_{6}\right)$ of the product of 1,2-hydroboration of pyrazine catalyzed by 1 . 


\section{1,3-bis (4,4,5,5-tetramethyl-1,3,2-dioxaborolan-2-yl)-1,2,3,4-tetrahydropyrimidine (2q):}

Pale yellow oil (238 $\mathrm{mg}, 71 \%)$ :

${ }^{1} \mathrm{H}$ NMR $\left(500 \mathrm{MHz}, \mathrm{C}_{6} \mathrm{D}_{6}\right): 6.81(\mathrm{~d}, J=5.0 \mathrm{~Hz}, 1 \mathrm{H}), 4.80(\mathrm{~s}, 2 \mathrm{H}), 4.62(\mathrm{~m}, 1 \mathrm{H}), 3.80(\mathrm{~s}, 2 \mathrm{H})$, $1.05(\mathrm{~s}, 12 \mathrm{H}), 1.00(\mathrm{~s}, 12 \mathrm{H}) \mathrm{ppm}$.

${ }^{13} \mathrm{C}$ NMR (125 MHz, $\left.\mathrm{C}_{6} \mathrm{D}_{6}\right)$ : 136.8, 109.6, 82.6, 82.1, 57.2, 42.2, 24.3, $24.2 \mathrm{ppm}$.

Anal. Calc. for $\mathrm{C}_{16} \mathrm{H}_{30} \mathrm{~B}_{2} \mathrm{~N}_{2} \mathrm{O}_{4}$ : C, 57.19; H, 9.00; N, 8.34. Found: C, 57.18; H, 8.79; N, 8.30.

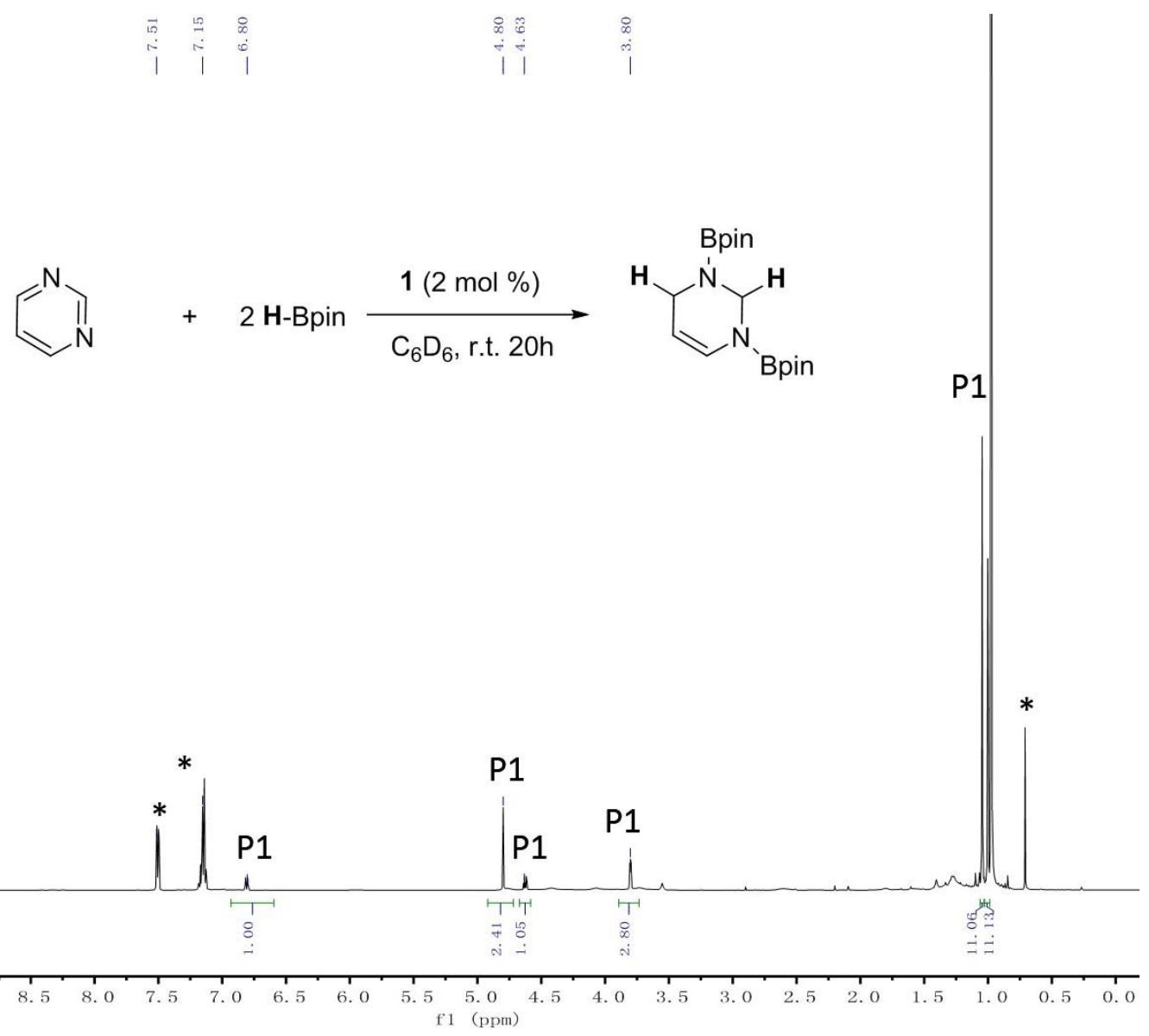

Figure S45. ${ }^{1} \mathrm{H}-\mathrm{NMR}$ spectrum $\left(500 \mathrm{MHz}, \mathrm{C}_{6} \mathrm{D}_{6}\right)$ of the product of 1,2-hydroboration of pyrimidine catalyzed by 1 . Assignment: $\mathrm{P} 1=$ predominant product; * internal standard (triphenylmethylsilane). 


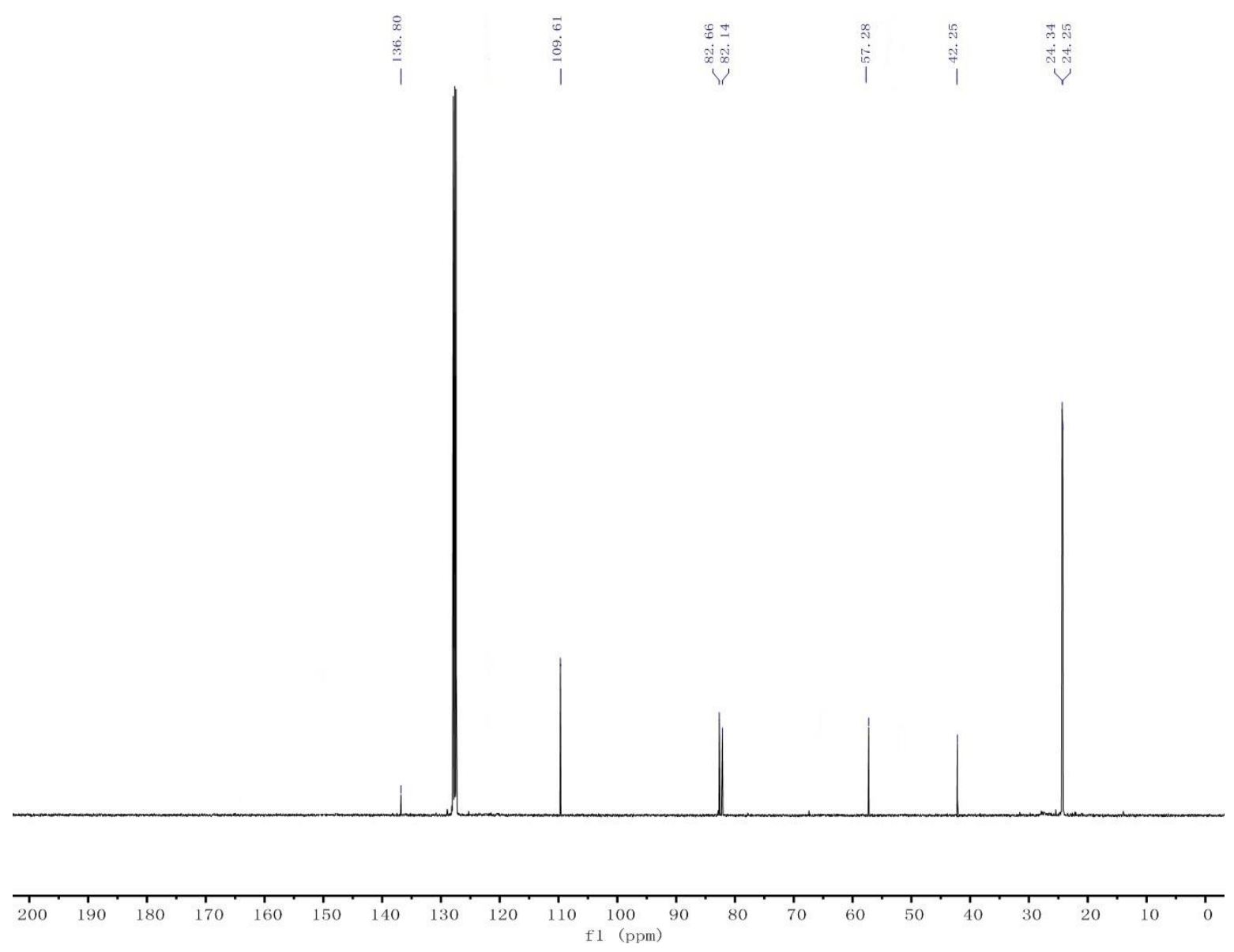

Figure S46. ${ }^{13} \mathrm{C}-\mathrm{NMR}$ spectrum $\left(125 \mathrm{MHz}, \mathrm{C}_{6} \mathrm{D}_{6}\right)$ of the product of 1,2-hydroboration of pyrimidine catalyzed by $\mathbf{1}$. 
2,5-di-Me-1,4-bis(4,4,5,5-tetramethyl-1,3,2-dioxaborolan-2-yl)-1,2,3,4-tetrahydropyrazine (2r):

Colorless oil (201 mg, 55\%):

${ }^{1} \mathrm{H}$ NMR (500 MHz, $\left.\mathrm{C}_{6} \mathrm{D}_{6}\right): 6.00(\mathrm{~s}, 1 \mathrm{H}), 3.98(\mathrm{q}, J=10.0 \mathrm{~Hz}, 1 \mathrm{H}), 3.68(\mathrm{~d}, J=10.0 \mathrm{~Hz}, 1 \mathrm{H})$, $3.06(\mathrm{dd}, J=15.0 \mathrm{~Hz}, 1 \mathrm{H}), 2.11(\mathrm{~s}, 3 \mathrm{H}), 1.25(\mathrm{~d}, J=10.0 \mathrm{~Hz}, 3 \mathrm{H}), 1.08(\mathrm{~s}, 12 \mathrm{H}), 1.05(\mathrm{~s}, 12 \mathrm{H})$ ppm.

${ }^{13} \mathrm{C}$ NMR (125 MHz, $\left.\mathrm{C}_{6} \mathrm{D}_{6}\right): 116.3,109.4,82.1,47.1,46.0,24.5,24.4,24.3,24.2,23.8,19.2$ ppm.

Anal. Calc. for $\mathrm{C}_{18} \mathrm{H}_{34} \mathrm{~B}_{2} \mathrm{~N}_{2} \mathrm{O}_{4}$ : C, 59.38; H, 9.41; N, 7.69. Found: C, 59.34; H, 9.29; N, 7.68.
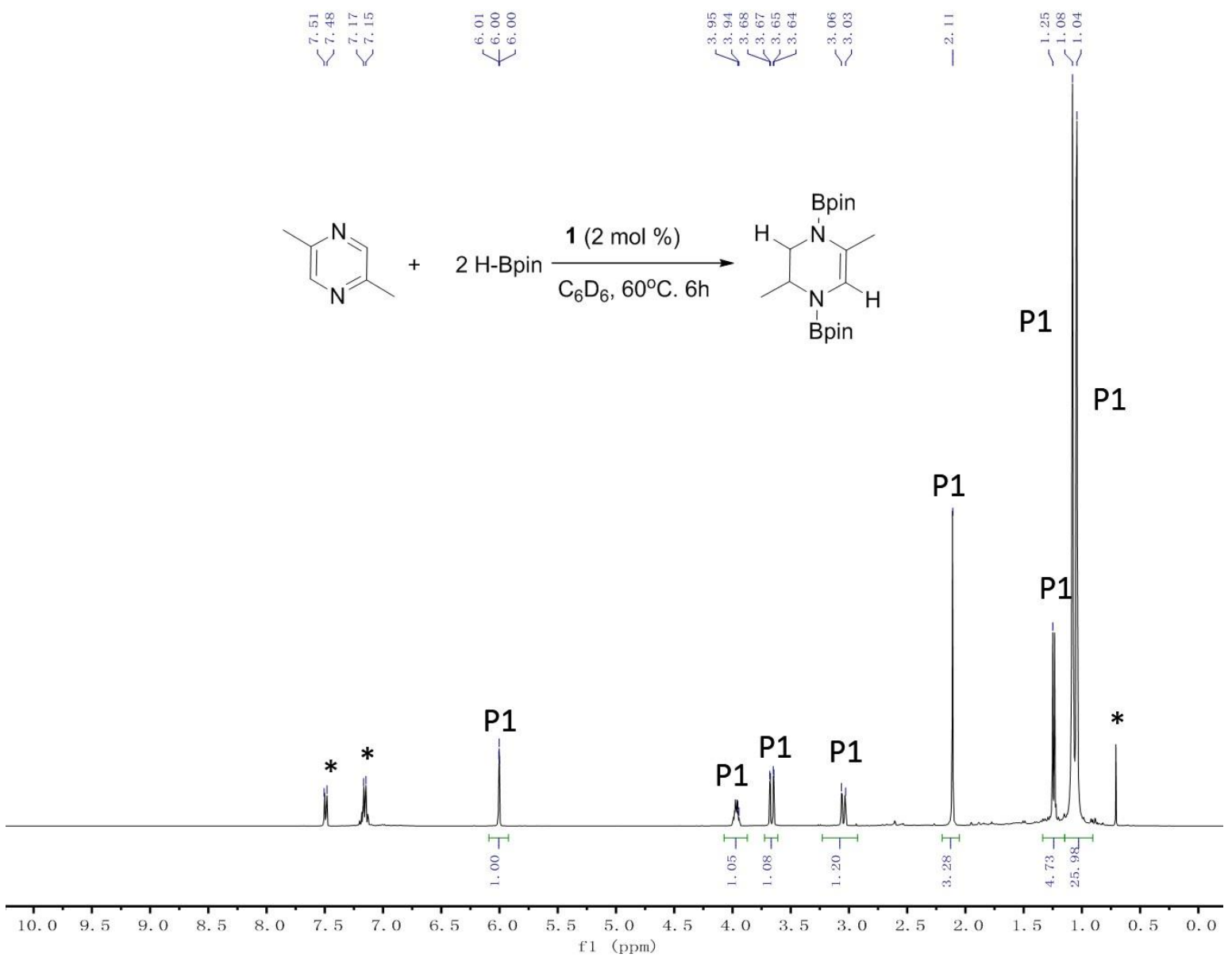

Figure S47. ${ }^{1} \mathrm{H}-\mathrm{NMR}$ spectrum $\left(500 \mathrm{MHz}, \mathrm{C}_{6} \mathrm{D}_{6}\right)$ of the product of hydroboration of 2,5-di-Mepyrazine catalyzed by 1. Assignment: $\mathrm{P} 1=$ predominant product; * internal standard (triphenylmethylsilane). 

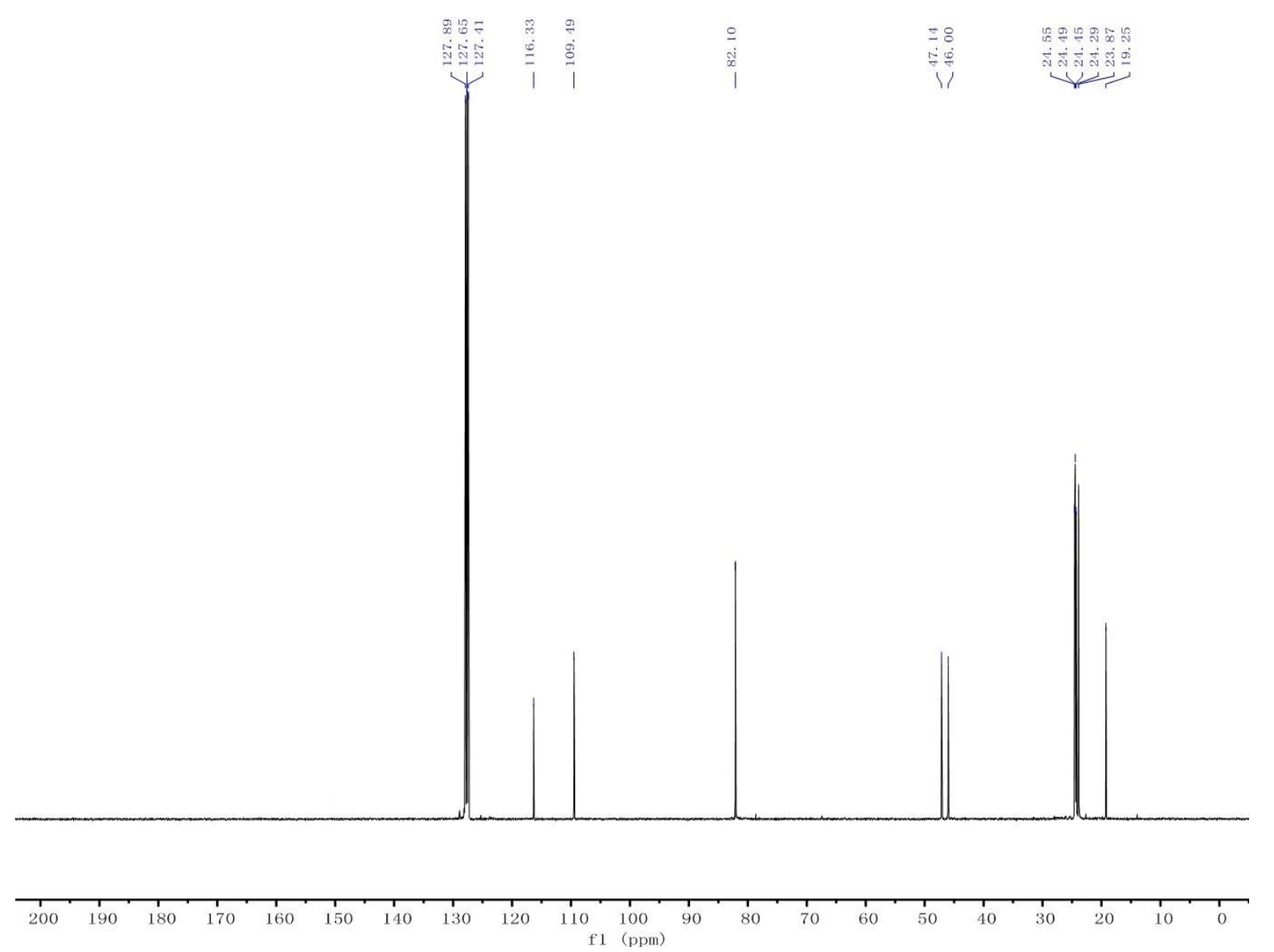

Figure S48. ${ }^{13} \mathrm{C}-\mathrm{NMR}$ spectrum $\left(125 \mathrm{MHz}, \mathrm{C}_{6} \mathrm{D}_{6}\right)$ of the product of 1,2-hydroboration of 2,5-diMe-pyrazine catalyzed by 1 . 
1,4-bis(4,4,5,5-tetramethyl-1,3,2-dioxaborolan-2-yl)-1,4-dihydroquinoxaline (2s):

White solid (341 mg, 89\%):

${ }^{1} \mathrm{H}$ NMR (500 MHz, C $\left.6 \mathrm{D}_{6}\right): 8.14(\mathrm{~d}, J=5.0 \mathrm{~Hz}, 2 \mathrm{H}), 6.96(\mathrm{~d}, J=10.0 \mathrm{~Hz}, 2 \mathrm{H}), 3.61(\mathrm{~s}, 4 \mathrm{H})$, $1.01(\mathrm{~s}, 24 \mathrm{H}) \mathrm{ppm}$.

${ }^{13} \mathrm{C}$ NMR (125 MHz, $\left.\mathrm{C}_{6} \mathrm{D}_{6}\right): 132.8,121.1,120.7,82.3,43.8,24.3 \mathrm{ppm}$.

Anal. Calc. for $\mathrm{C}_{20} \mathrm{H}_{30} \mathrm{~B}_{2} \mathrm{~N}_{2} \mathrm{O}_{4}$ : C, 62.54; H, 7.87; N, 7.29. Found: C, 62.51; H, 7.71; N, 7.27.
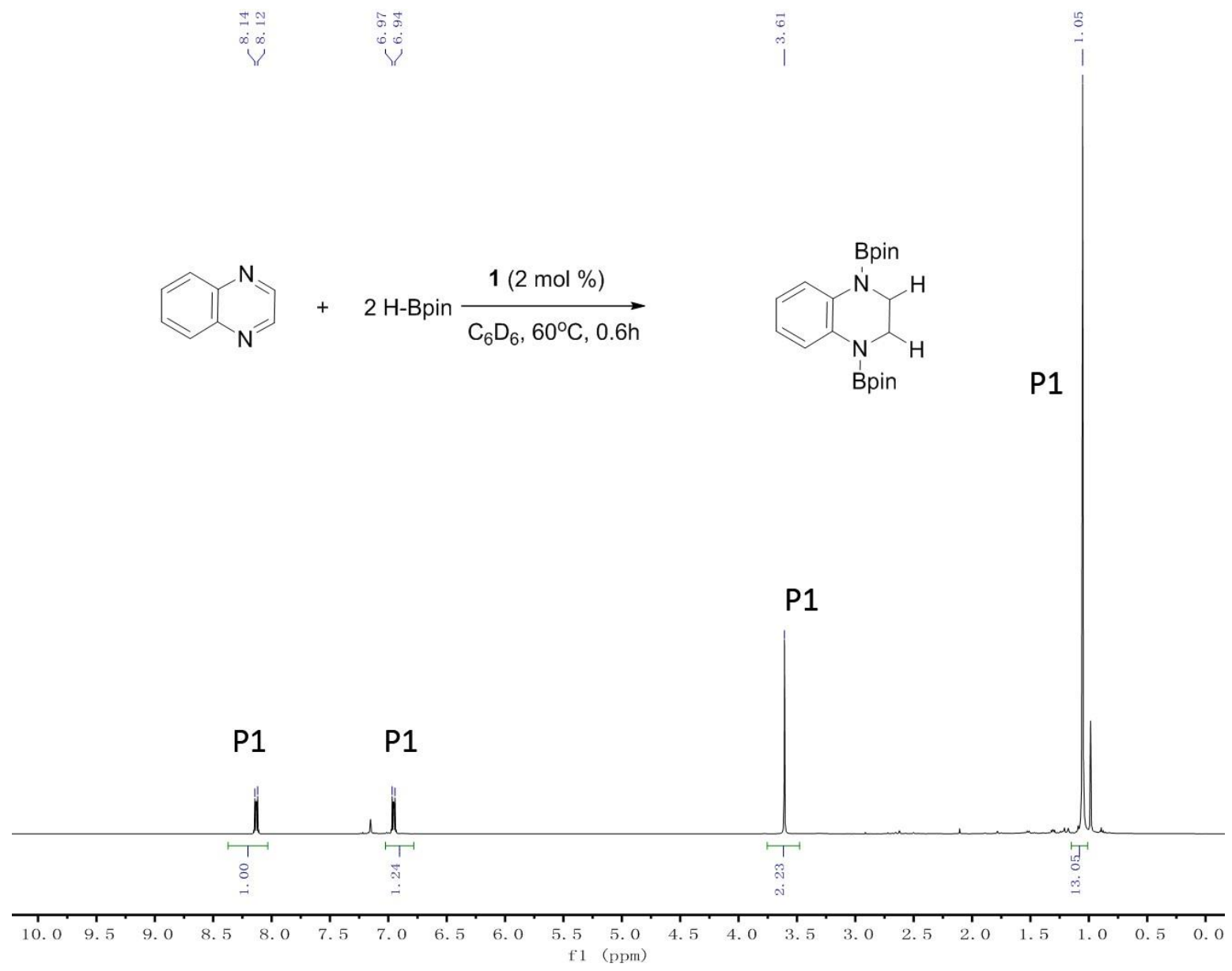

Figure S49. ${ }^{1} \mathrm{H}-\mathrm{NMR}$ spectrum $\left(500 \mathrm{MHz}, \mathrm{C}_{6} \mathrm{D}_{6}\right)$ of the product of hydroboration of quinoxaline catalyzed by 1 . Assignment: $\mathrm{P} 1$ = predominant product. 


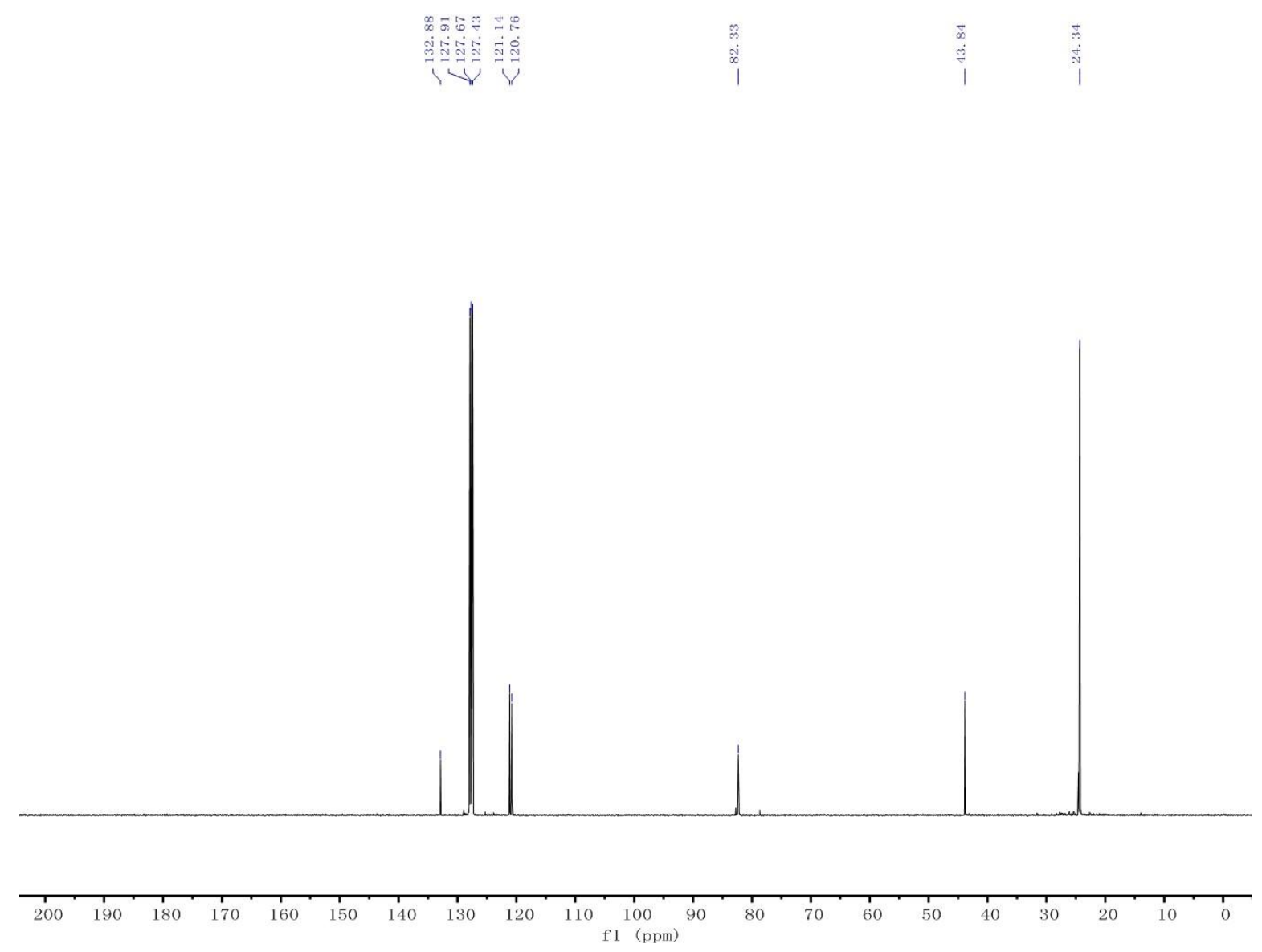

Figure S50. ${ }^{13} \mathrm{C}-\mathrm{NMR}$ spectrum $\left(125 \mathrm{MHz}, \mathrm{C}_{6} \mathrm{D}_{6}\right)$ of the product of 1,2-hydroboration of quinoxaline catalyzed by 1 . 


\section{2-(1-((4,4,5,5-tetramethyl-1,3,2-dioxaborolan-2-yl)oxy)ethyl)pyridine (2t):}

White crystal solid (239 mg, 96\%):

${ }^{1} \mathrm{H}$ NMR $\left(500 \mathrm{MHz}, \mathrm{C}_{6} \mathrm{D}_{6}\right): 8.38(\mathrm{td}, J=5.0 \mathrm{~Hz}, 1 \mathrm{H}), 7.31(\mathrm{dd}, J=10.0 \mathrm{~Hz}, 1 \mathrm{H}), 7.10(\mathrm{dd}, J=$ $10.0 \mathrm{~Hz}, 1 \mathrm{H}), 6.58(\mathrm{dd}, J=10.0 \mathrm{~Hz}, 1 \mathrm{H}), 5.57(\mathrm{q}, J=10.0 \mathrm{~Hz}, 1 \mathrm{H}), 1.61(\mathrm{~d}, J=5.0 \mathrm{~Hz}, 3 \mathrm{H})$, $1.10(\mathrm{~s}, 12 \mathrm{H}), 0.99(\mathrm{~s}, 12 \mathrm{H}) \mathrm{ppm}$.

${ }^{13} \mathrm{C}$ NMR (125 MHz, $\left.\mathrm{C}_{6} \mathrm{D}_{6}\right): 163.8,147.0,136.6,121.7,118.7,81.7,73.2,24.6 \mathrm{ppm}$.

Anal. Calc. for $\mathrm{C}_{13} \mathrm{H}_{20} \mathrm{BNO}_{3}$ : C, 62.68; H, 8.09; N, 5.62. Found: C, 62.67; H, 7.97; N, 5.70.

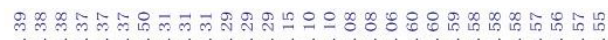

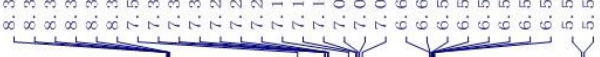

$\underset{O}{\stackrel{H}{\longrightarrow}} \stackrel{1(2 \mathrm{~mol} \%)}{\mathrm{C}_{6} \mathrm{D}_{6}, \text { r.t. } 2 \mathrm{~min}}$

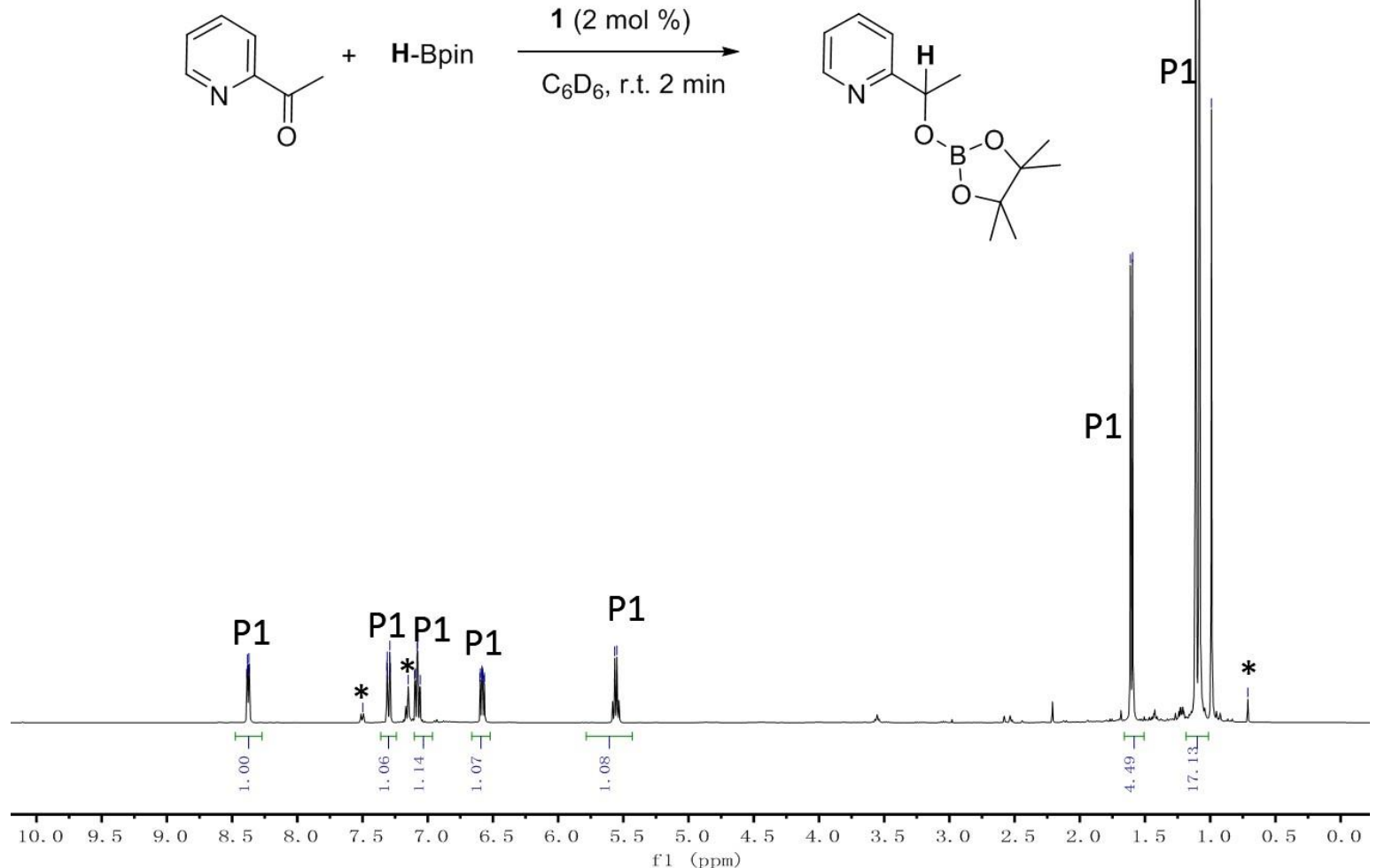

Figure S51. ${ }^{1} \mathrm{H}-\mathrm{NMR}$ spectrum $\left(500 \mathrm{MHz}, \mathrm{C}_{6} \mathrm{D}_{6}\right)$ of the product of 1,2-hydroboration of 1(pyridin-2-yl)ethan-1-one catalyzed by 1 . Assignment: $\mathrm{P} 1=$ predominant product; $*$ internal standard (triphenylmethylsilane). 


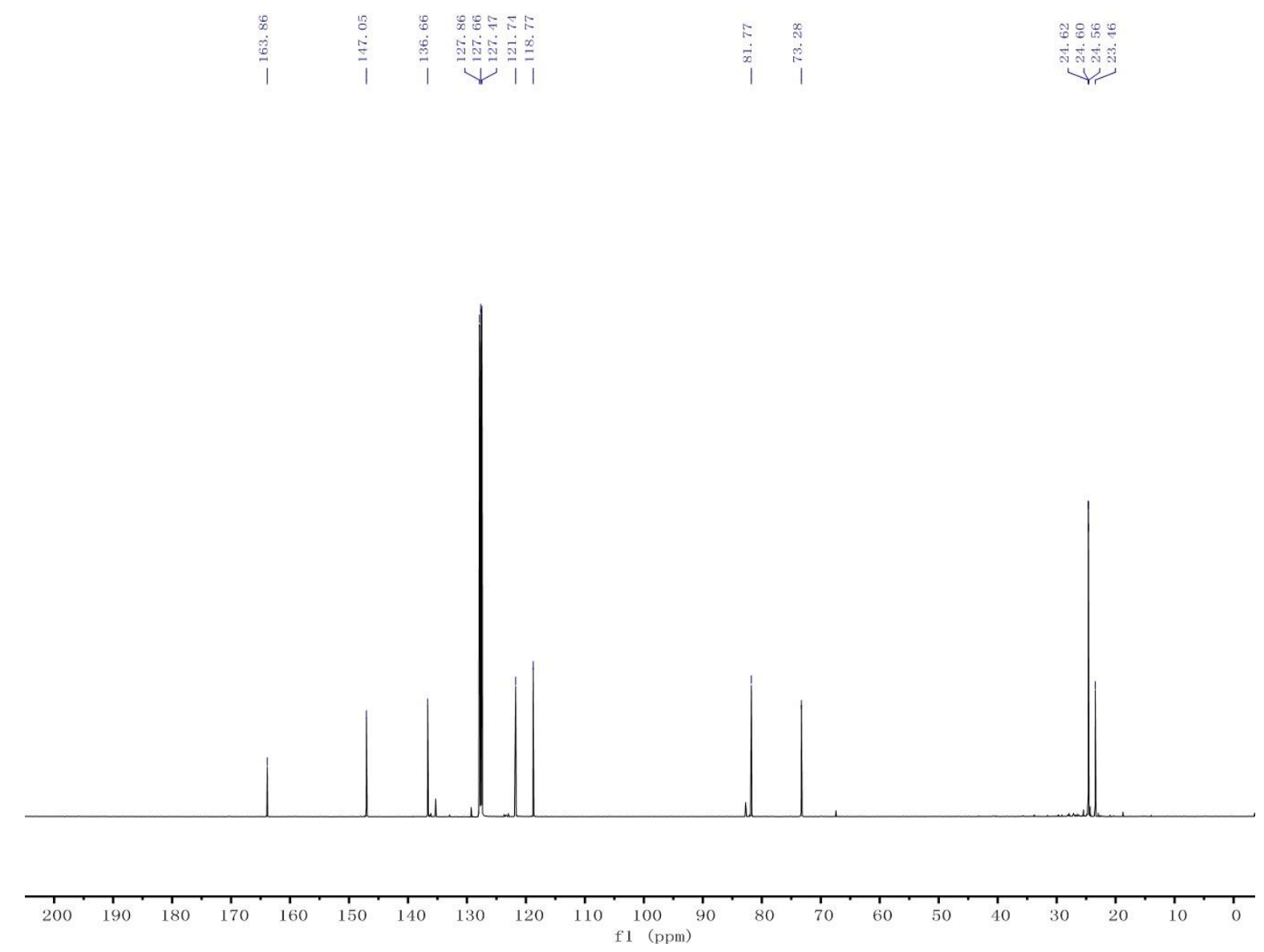

Figure S52. ${ }^{13} \mathrm{C}-\mathrm{NMR}$ spectrum $\left(125 \mathrm{MHz}, \mathrm{C}_{6} \mathrm{D}_{6}\right)$ of the product of 1,2-hydroboration of 1(pyridin-2-yl)ethan-1-one catalyzed by $\mathbf{1}$. 


\section{3-(((4,4,5,5-tetramethyl-1,3,2-dioxaborolan-2-yl)oxy)methyl)pyridine (2u):}

White solid (225 mg, 96\%):

${ }^{1} \mathrm{H}$ NMR $\left(500 \mathrm{MHz}, \mathrm{C}_{6} \mathrm{D}_{6}\right): 8.36(\mathrm{~s}, 1 \mathrm{H}), 8.18(\mathrm{dd}, J=10.0 \mathrm{~Hz}, 1 \mathrm{H}), 7.18(\mathrm{dd}, J=10.0 \mathrm{~Hz}, 1 \mathrm{H})$, 6.67 (dd, $J=10.0 \mathrm{~Hz}, 1 \mathrm{H}), 4.52(\mathrm{~s}, 2 \mathrm{H}), 0.81$ (s, 12H) ppm.

${ }^{13} \mathrm{C}$ NMR (125 MHz, $\left.\mathrm{C}_{6} \mathrm{D}_{6}\right):$ : 149.0, 148.8, 134.5, 133.8, 122.8, 82.5, 64.2, $24.2 \mathrm{ppm}$.

Anal. Calc. for $\mathrm{C}_{12} \mathrm{H}_{18} \mathrm{BNO}_{3}$ : C, 61.31; H, 7.72; N, 5.96. Found: C, 61.28; H, 7.60; N, 5.91.

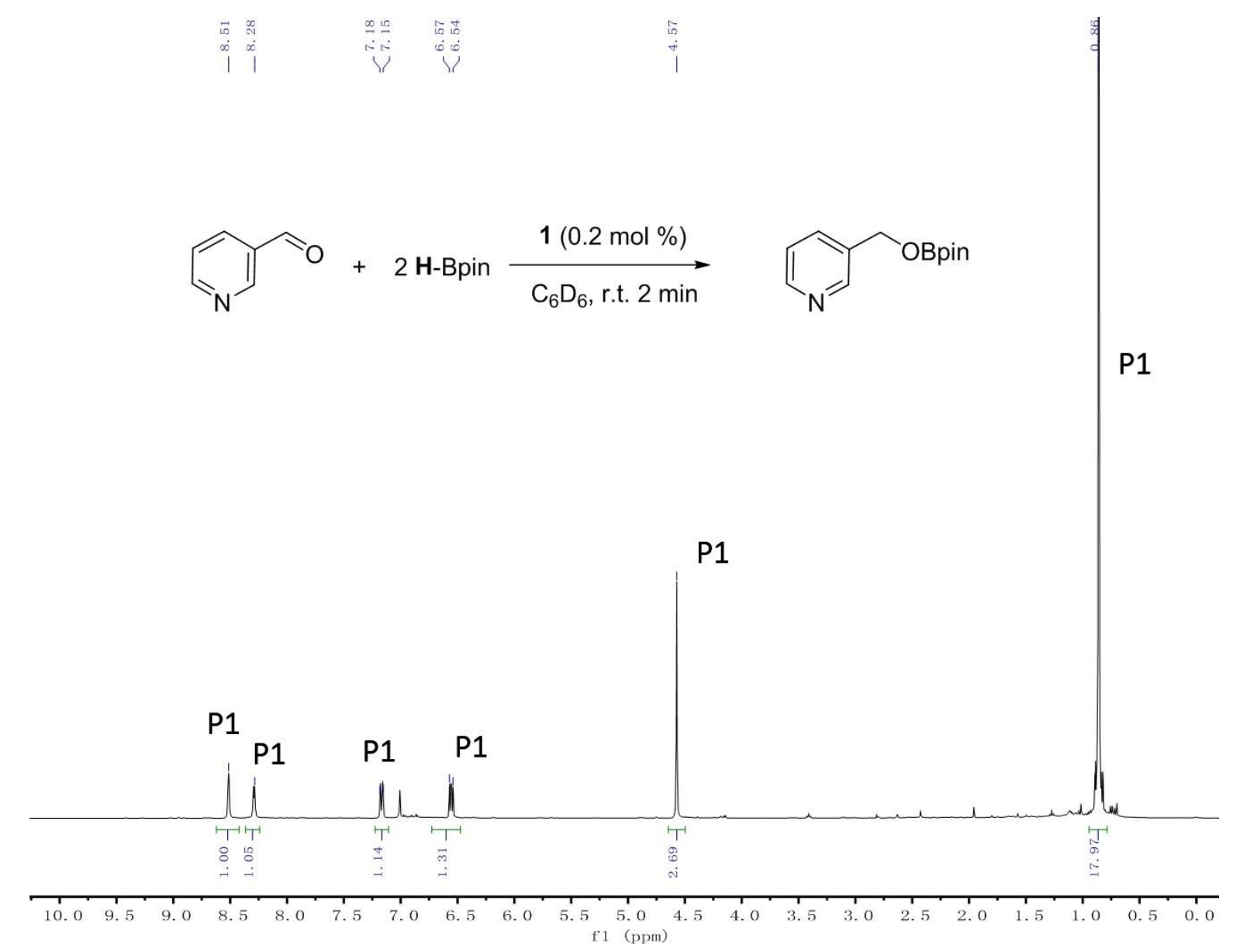

Figure S53. ${ }^{1} \mathrm{H}-\mathrm{NMR}$ spectrum $\left(500 \mathrm{MHz}, \mathrm{C}_{6} \mathrm{D}_{6}\right)$ of the product of 1,2-hydroboration of nicotinaldehyde catalyzed by $\mathbf{1}$. Assignment: $\mathrm{P} 1$ = predominant product. 


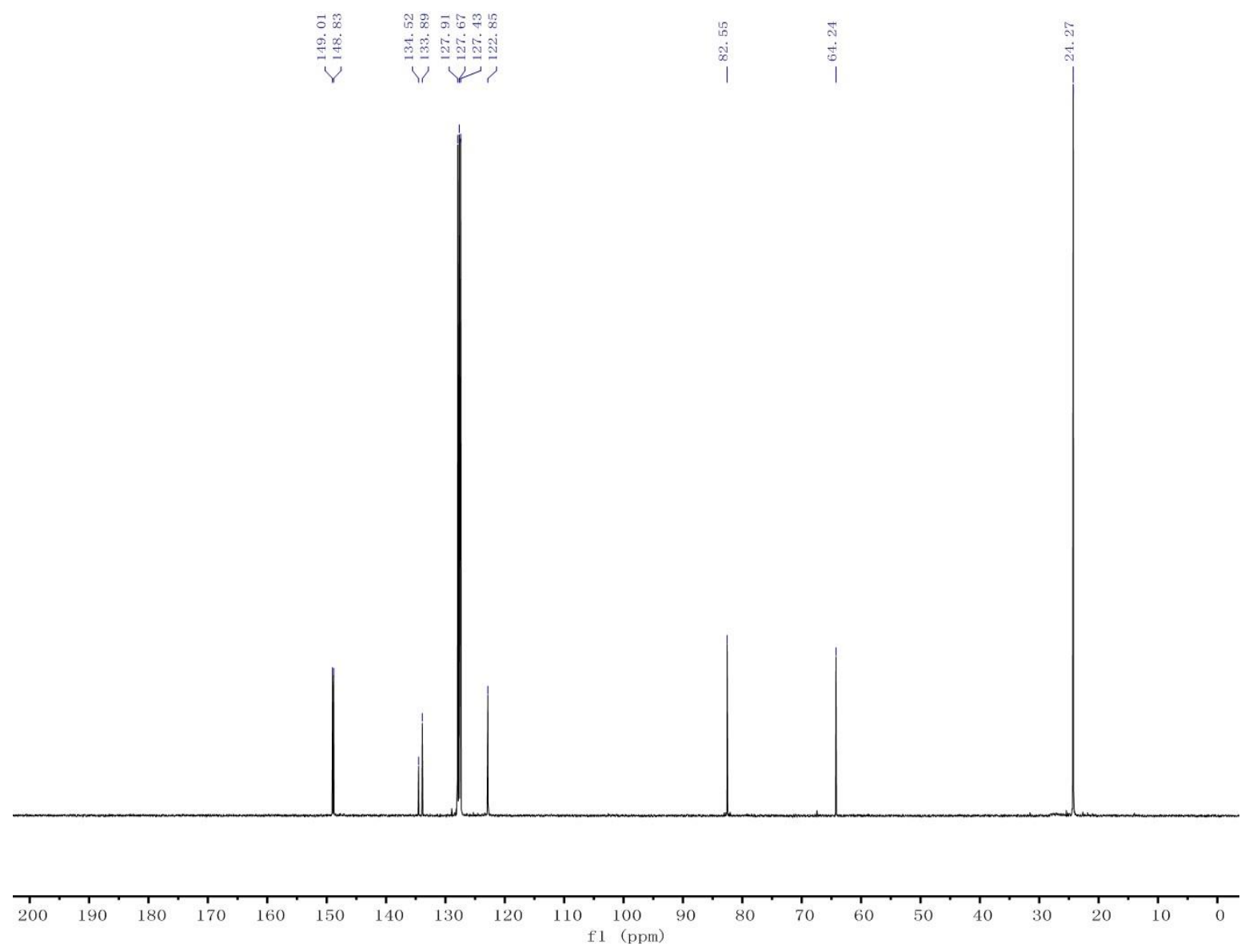

Figure S54. ${ }^{13} \mathrm{C}-\mathrm{NMR}$ spectrum $\left(125 \mathrm{MHz}, \mathrm{C}_{6} \mathrm{D}_{6}\right)$ of the product of 1,2-hydroboration of nicotinaldehyde catalyzed by 1 . 


\section{3-(((4,4,5,5-tetramethyl-1,3,2-dioxaborolan-2-yl)oxy)methyl)pyridine (2v):}

White solid for P1 (195 mg, 83\%):

${ }^{1} \mathrm{H}$ NMR $\left(500 \mathrm{MHz}, \mathrm{C}_{6} \mathrm{D}_{6}\right): 8.64(\mathrm{~s}, 1 \mathrm{H}), 8.43(\mathrm{dd}, J=10.0 \mathrm{~Hz}, 1 \mathrm{H}), 7.16(\mathrm{dd}, J=10.0 \mathrm{~Hz}, 1 \mathrm{H})$, $6.65(\mathrm{dd}, J=10.0 \mathrm{~Hz}, 1 \mathrm{H}), 4.71(\mathrm{~s}, 2 \mathrm{H}), 3.48(\mathrm{~s}, 3 \mathrm{H}), 1.05(\mathrm{~s}, 12 \mathrm{H}), 0.99$ (s, 12H) ppm.

${ }^{13} \mathrm{C}$ NMR (125 MHz, $\left.\mathrm{C}_{6} \mathrm{D}_{6}\right): 148.7,148.5,134.5,134.0,122.9,82.5,67.3,64.2,25.4,24.6,24.2$ ppm.

Anal. Calc. for $\mathrm{C}_{12} \mathrm{H}_{18} \mathrm{BNO}_{3}$ : C, 61.31; H, 7.72; N, 5.96. Found: C, 61.30; H, 7.61; N, 5.96.

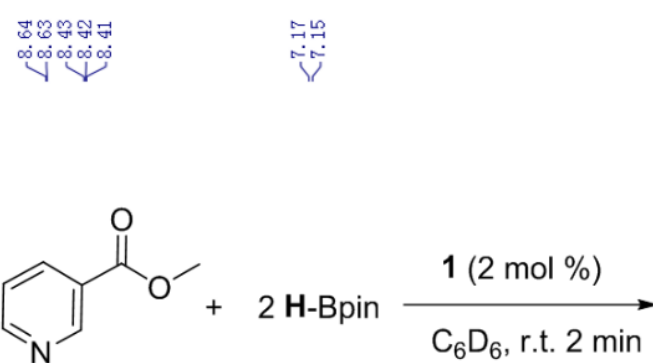<smiles></smiles>

P1

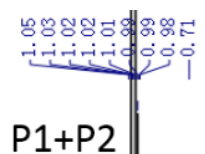

$\mathrm{P} 1+\mathrm{P} 2$
$\mathrm{CH}_{3} \mathrm{OBpin}$

P2

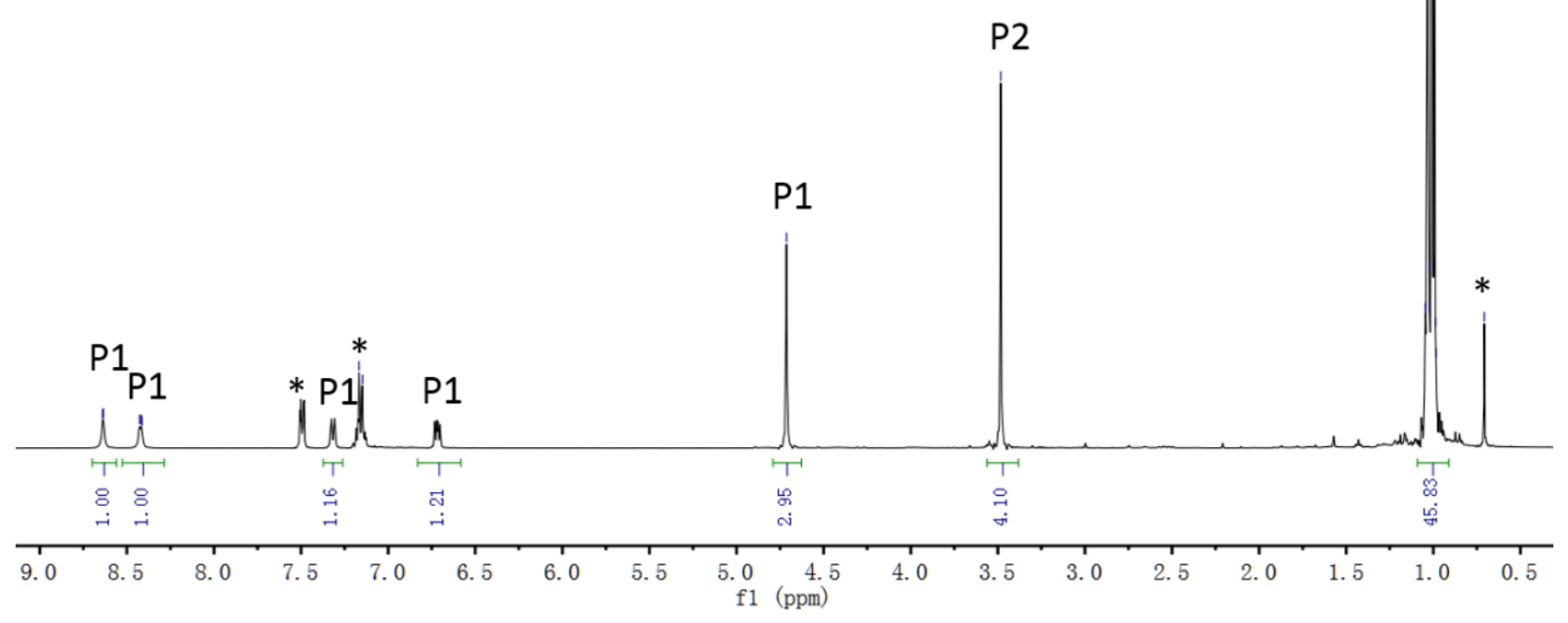

Figure S55. ${ }^{1} \mathrm{H}-\mathrm{NMR}$ spectrum $\left(500 \mathrm{MHz}, \mathrm{C}_{6} \mathrm{D}_{6}\right)$ of the product of 1,2-hydroboration of methyl nicotinate catalyzed by 1 . Assignment: $\mathrm{P} 1=$ predominant product; $\mathrm{P} 2=2$-methoxy-4,4,5,5tetramethyl-1,3,2-dioxaborolane; * internal standard (triphenylmethylsilane). 


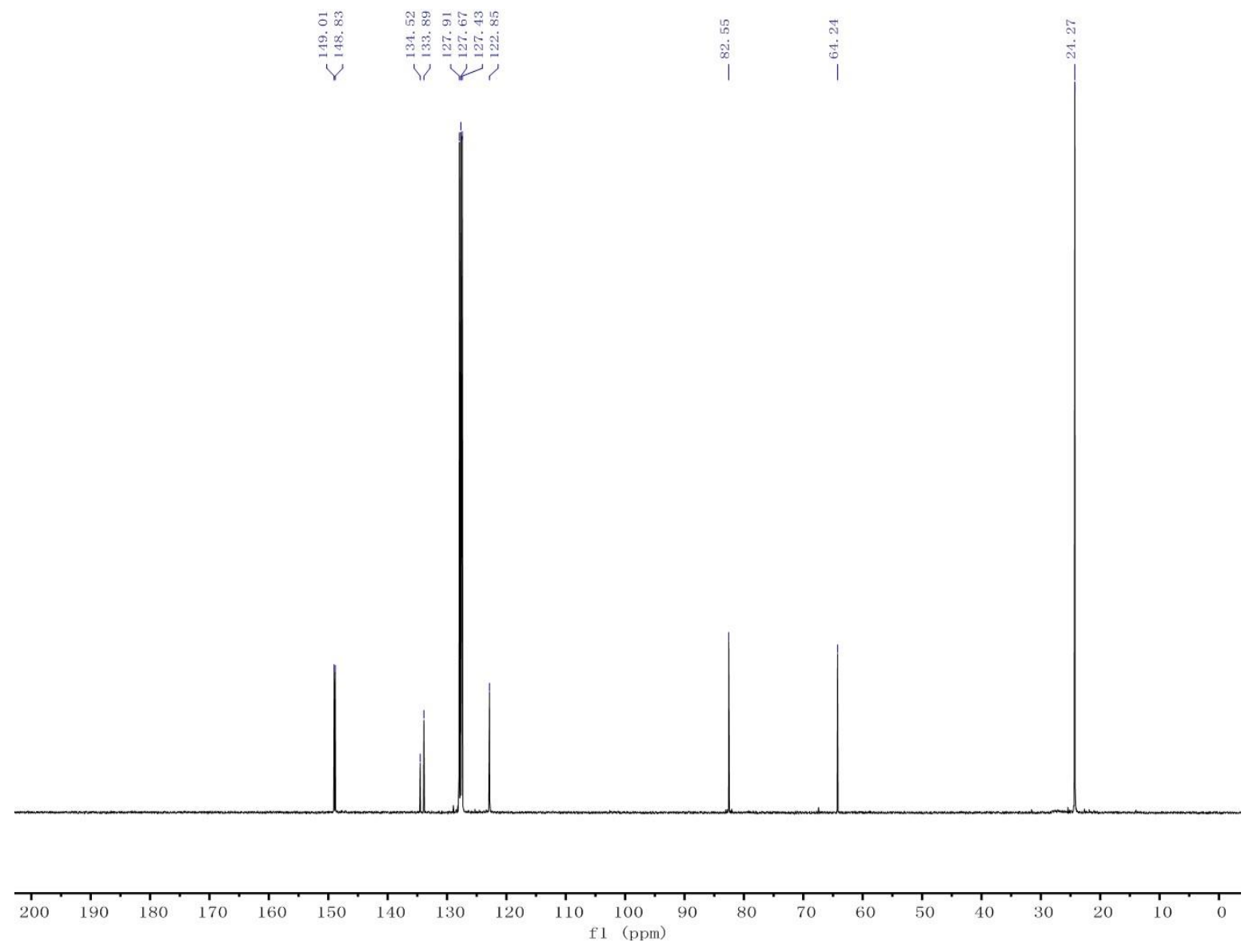

Figure S56. ${ }^{13} \mathrm{C}-\mathrm{NMR}$ spectrum $\left(125 \mathrm{MHz}, \mathrm{C}_{6} \mathrm{D}_{6}\right)$ of the product of 1,2-hydroboration of methyl nicotinate catalyzed by $\mathbf{1}$. 


\section{4-(((4,4,5,5-tetramethyl-1,3,2-dioxaborolan-2-yl)oxy)methyl)pyridine (2w):}

White solid for P1 (183 mg, 78\%):

${ }^{1} \mathrm{H}$ NMR (500 MHz, $\left.\mathrm{C}_{6} \mathrm{D}_{6}\right): 8.51(\mathrm{~s}, 2 \mathrm{H}), 6.90(\mathrm{~d}, J=5.0 \mathrm{~Hz}, 2 \mathrm{H}), 4.70(\mathrm{~s}, 2 \mathrm{H}), 3.51(\mathrm{~s}, 3 \mathrm{H})$, $1.05(\mathrm{~s}, 12 \mathrm{H}), 1.01(\mathrm{~s}, 12 \mathrm{H}) \mathrm{ppm}$.

${ }^{13} \mathrm{C}$ NMR (125 MHz, $\left.\mathrm{C}_{6} \mathrm{D}_{6}\right)$ : 149.8, 120.4, 82.6, 82.1, 64.8, 51.5, 24.3, $24.2 \mathrm{ppm}$.

Anal. Calc. for $\mathrm{C}_{12} \mathrm{H}_{18} \mathrm{BNO}_{3}$ : C, 61.31; H, 7.72; N, 5.96. Found: C, 61.28; H, 7.59; N, 5.95.

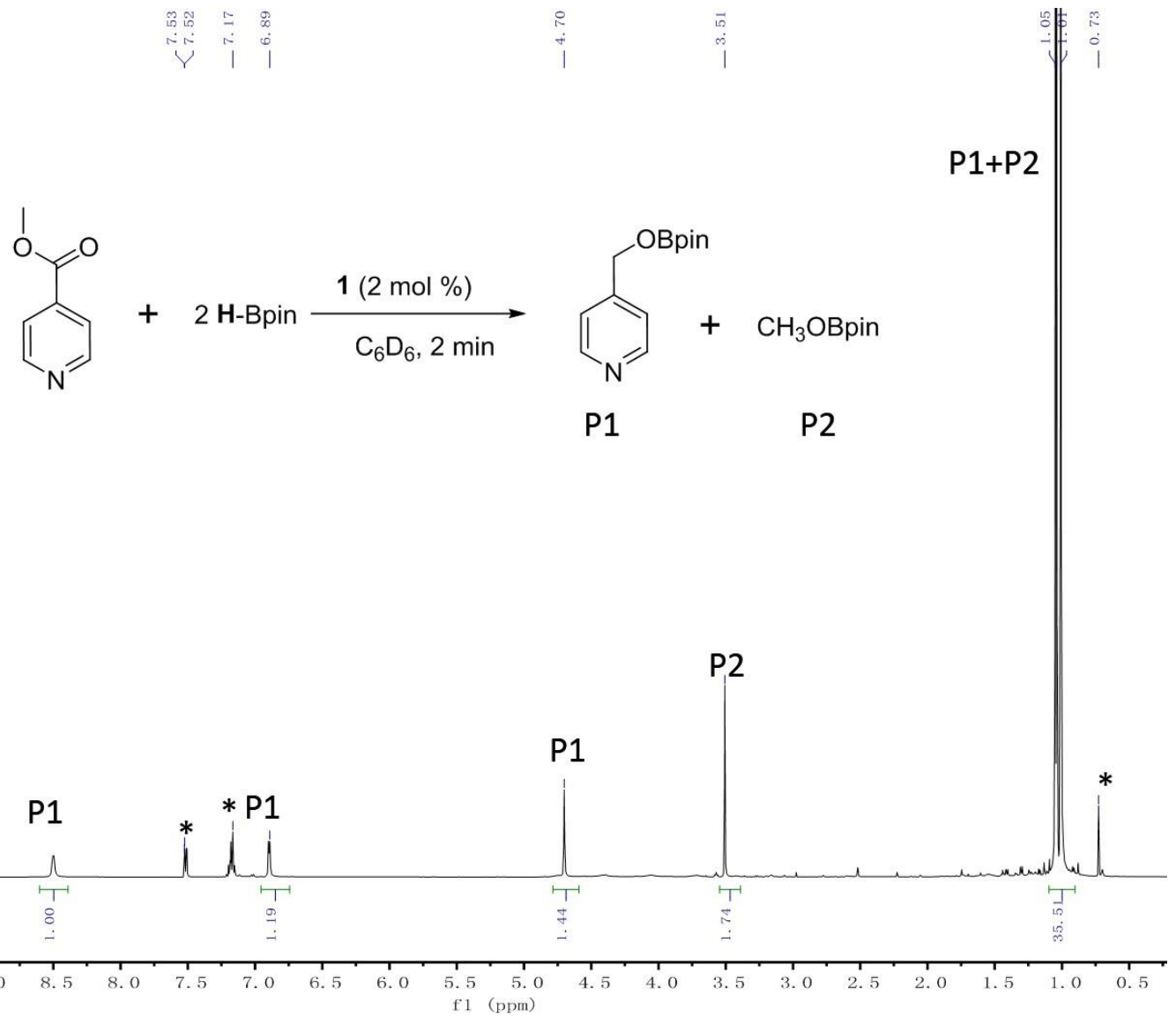

Figure S57. ${ }^{1} \mathrm{H}-\mathrm{NMR}$ spectrum $\left(500 \mathrm{MHz}, \mathrm{C}_{6} \mathrm{D}_{6}\right)$ of the product of 1,2-hydroboration of methyl isonicotinate catalyzed by 1 . Assignment: $\mathrm{P} 1$ = predominant product; $\mathrm{P} 2$ = 2-methoxy-4,4,5,5tetramethyl-1,3,2-dioxaborolane; * internal standard (triphenylmethylsilane). 

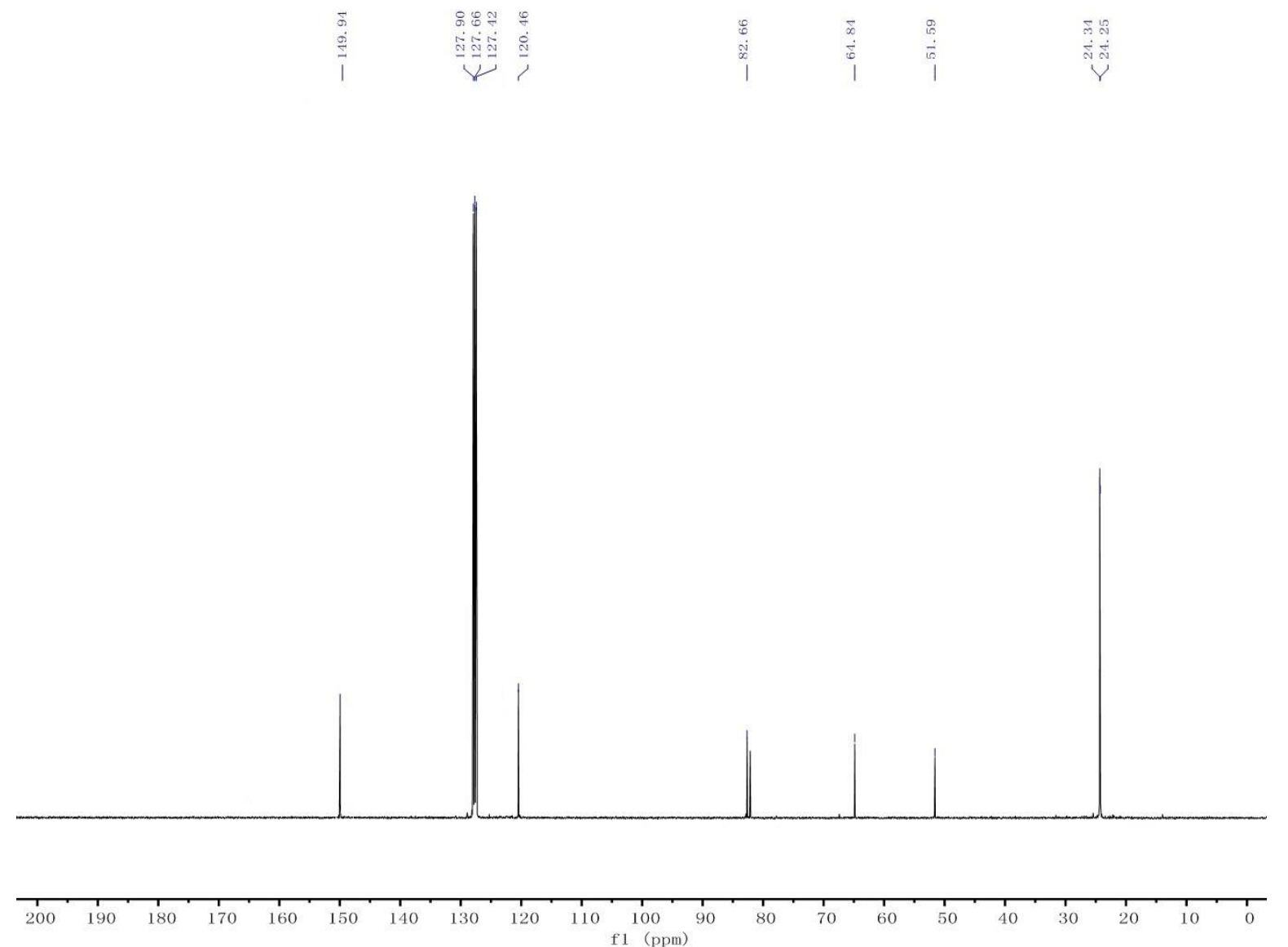

Figure S58. ${ }^{13} \mathrm{C}$-NMR spectrum $\left(125 \mathrm{MHz}, \mathrm{C}_{6} \mathrm{D}_{6}\right)$ of the product of 1,2-hydroboration of methyl isonicotinate catalyzed by $\mathbf{1}$. 
1.4.2 Characterization Data for Hydroboration Products by Catalysts 2 and 3

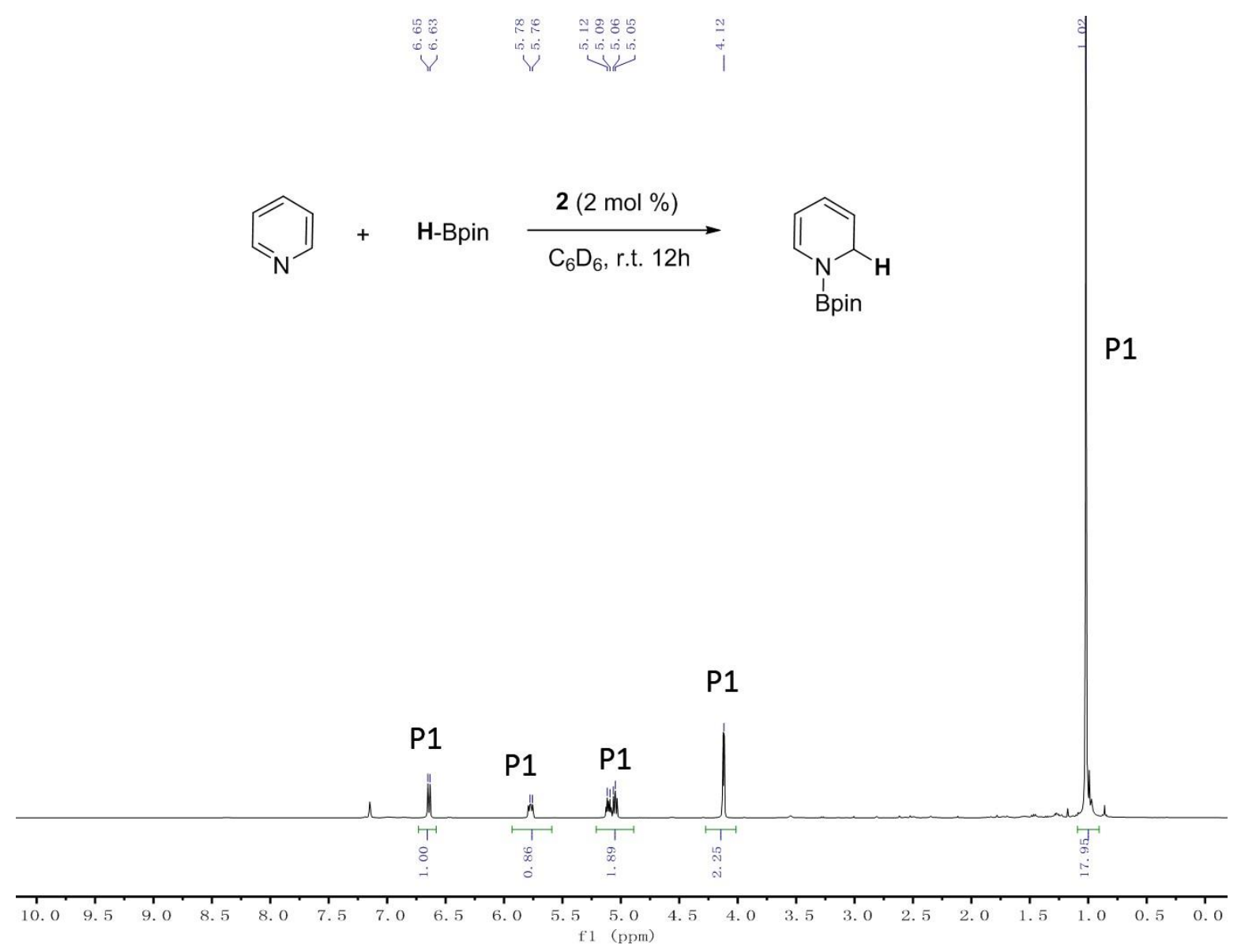

Figure S59. ${ }^{1} \mathrm{H}-\mathrm{NMR}$ spectrum $\left(500 \mathrm{MHz}, \mathrm{C}_{6} \mathrm{D}_{6}\right)$ of the catalytic hydroboration of pyridine based on $2\left(25^{\circ} \mathrm{C}, 12 \mathrm{~h}, 2 \%\right.$ catalyst loading $)$; (P1:P2 > 20:1). 


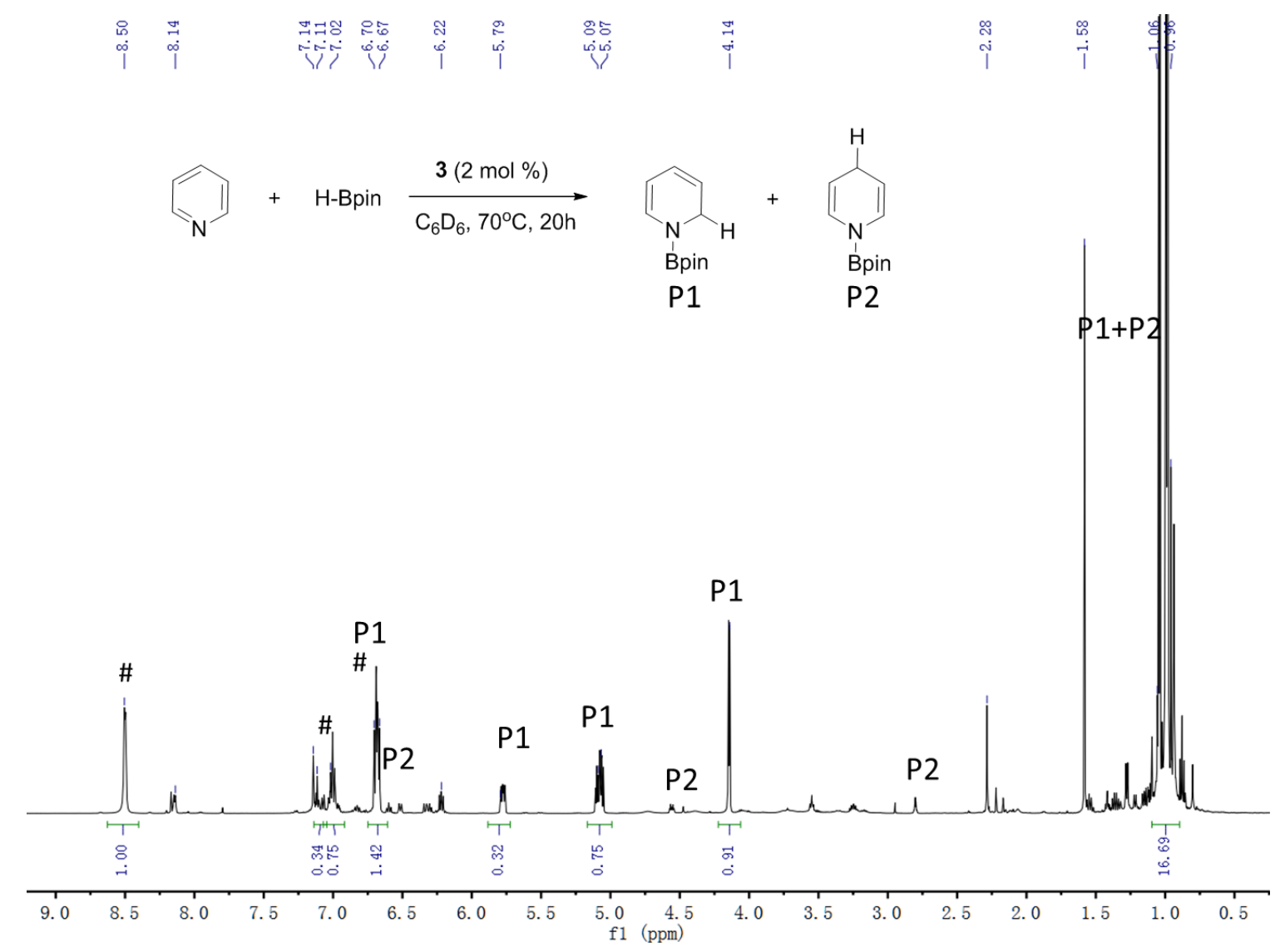

Figure S60. ${ }^{1} \mathrm{H}-\mathrm{NMR}$ spectrum $\left(500 \mathrm{MHz}, \mathrm{C}_{6} \mathrm{D}_{6}\right)$ of the catalytic hydroboration of pyridine based on $3\left(70^{\circ} \mathrm{C}, 20 \mathrm{~h}, 2 \%\right.$ catalyst loading); $(\mathrm{P} 1: \mathrm{P} 2=1.9: 1)$. \# unreacted pyridine. 
1.4.3 Stoichiometric Reaction of complex 1 with 2 equiv. of HBpin

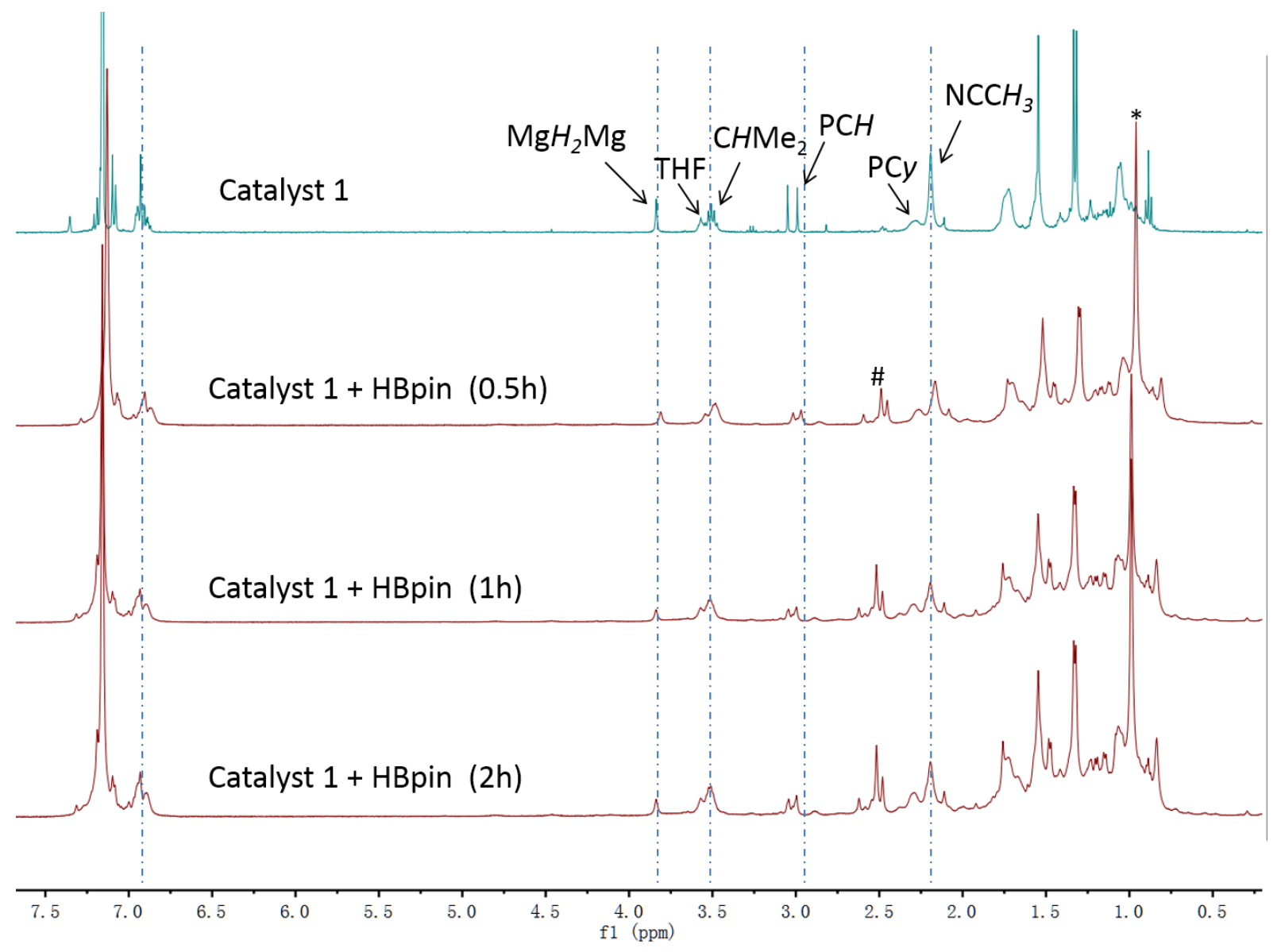

Figure S61. Stacking ${ }^{1} \mathrm{H}-\mathrm{NMR}$ spectra $\left(500 \mathrm{MHz}, \mathrm{C}_{6} \mathrm{D}_{6}\right)$ for monitoring the reaction of 1 with 2 equiv. of HBpin under regular time. ${ }^{\#}$ unidentified peak. 


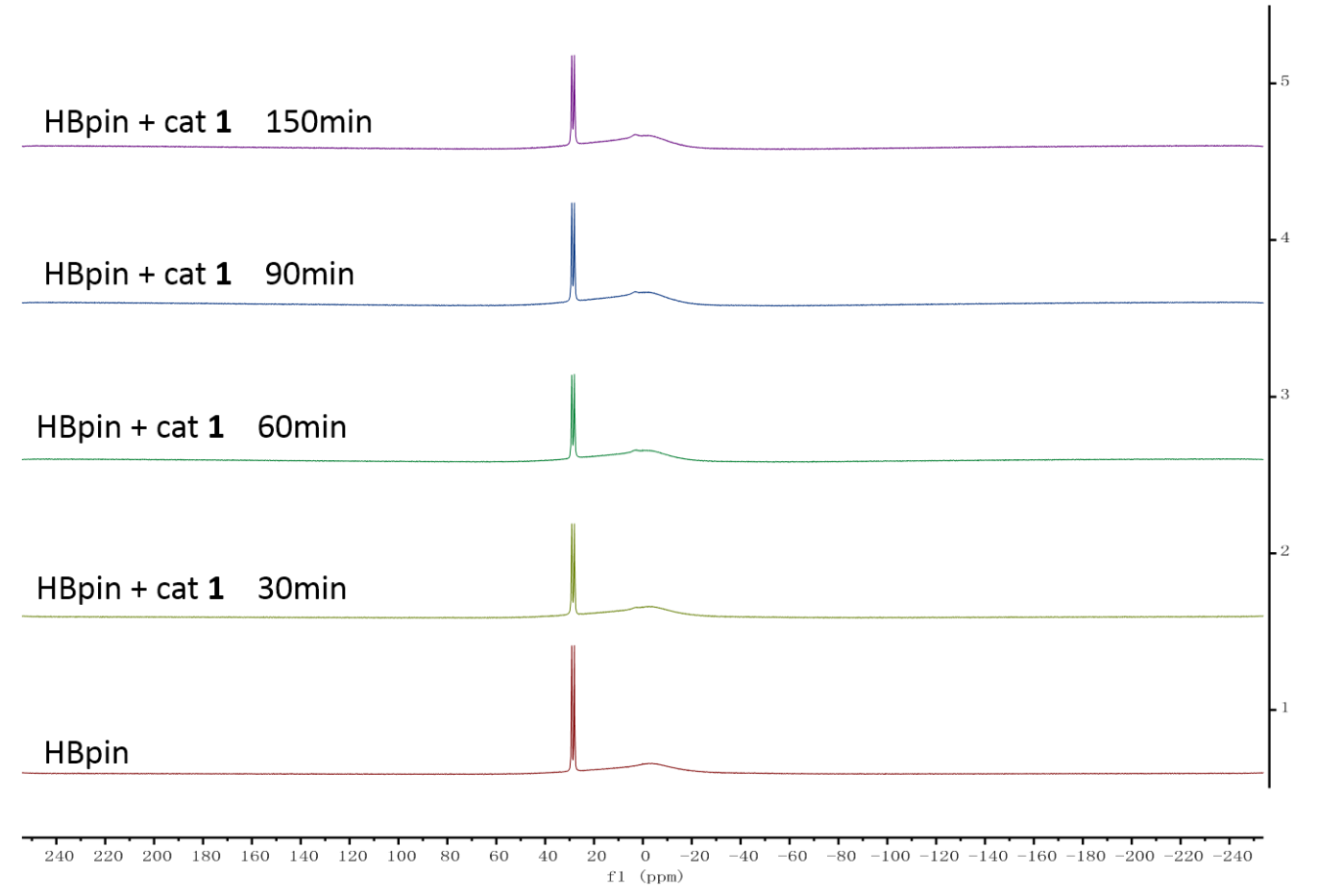

Figure S62. Stacking ${ }^{11}$ B-NMR spectra $\left(160.4 \mathrm{MHz}, \mathrm{C}_{6} \mathrm{D}_{6}\right)$ for monitoring the reaction of 1 with 2 equiv. of HBpin under regular time.

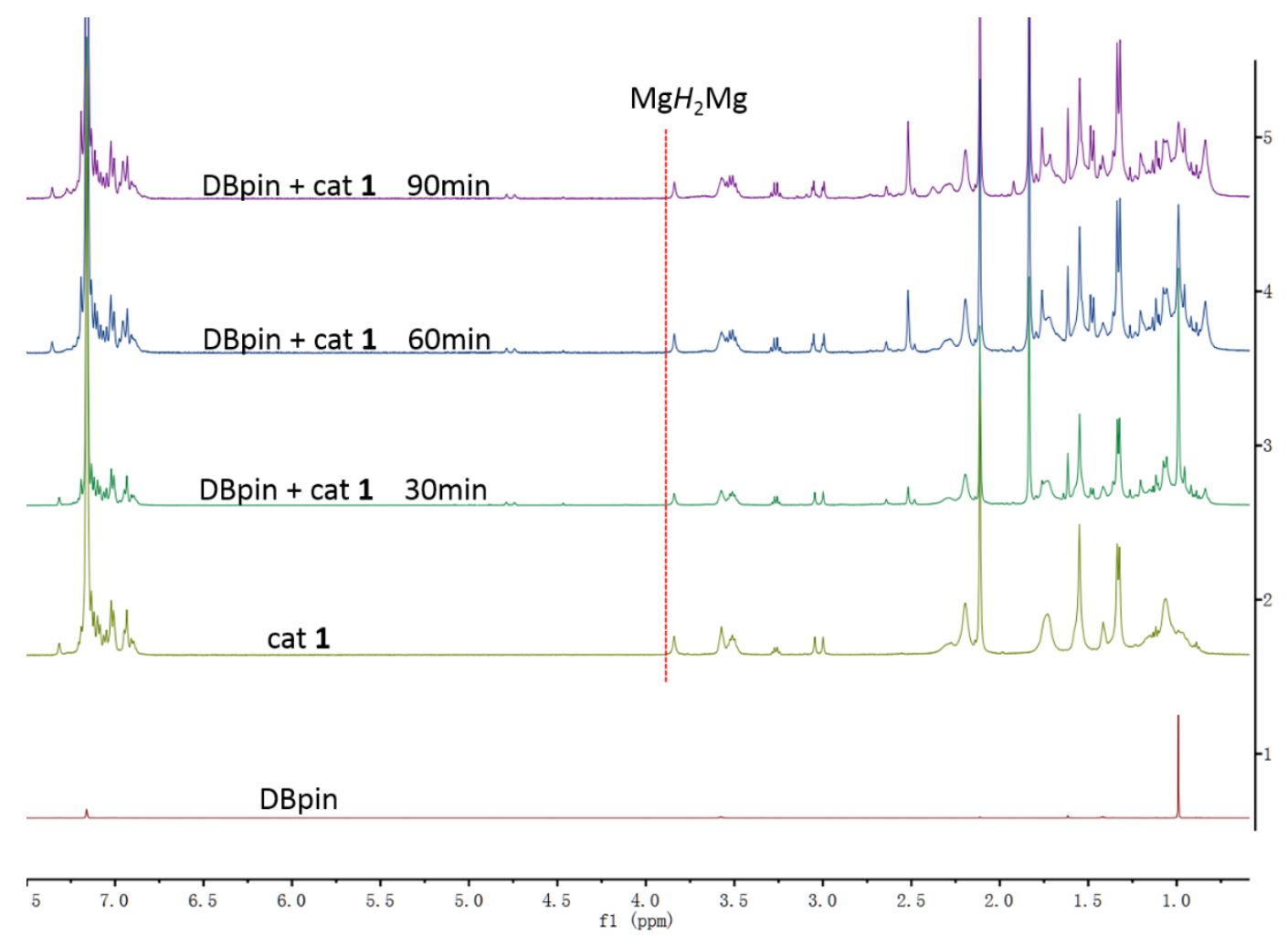

Figure S63. Stacking ${ }^{1} \mathrm{H}-\mathrm{NMR}$ spectra $\left(500 \mathrm{MHz}, \mathrm{C}_{6} \mathrm{D}_{6}\right)$ of monitoring the reaction of 1 with 2 equiv. of DBpin under regular time. 


\subsubsection{Stacking ${ }^{1} \mathrm{H}-\mathrm{NMR}$ Spectra for monitoring the initial reaction process}

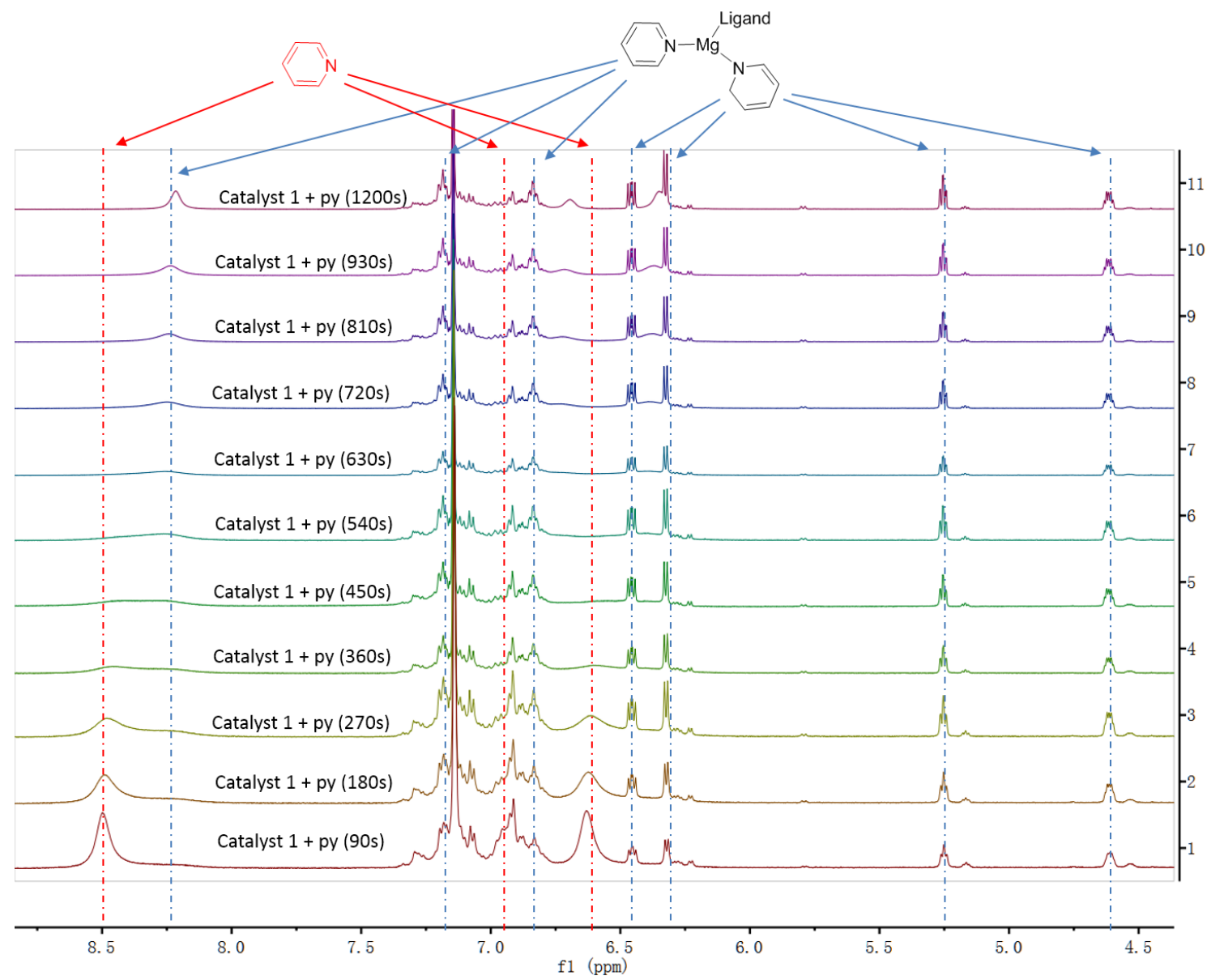

Figure S64. Stacking ${ }^{1} \mathrm{H}-\mathrm{NMR}$ spectra $\left(500 \mathrm{MHz}, \mathrm{C}_{6} \mathrm{D}_{6}\right)$ for monitoring the reaction of cat1 with 1 stoichiometric amount of pyridine. 


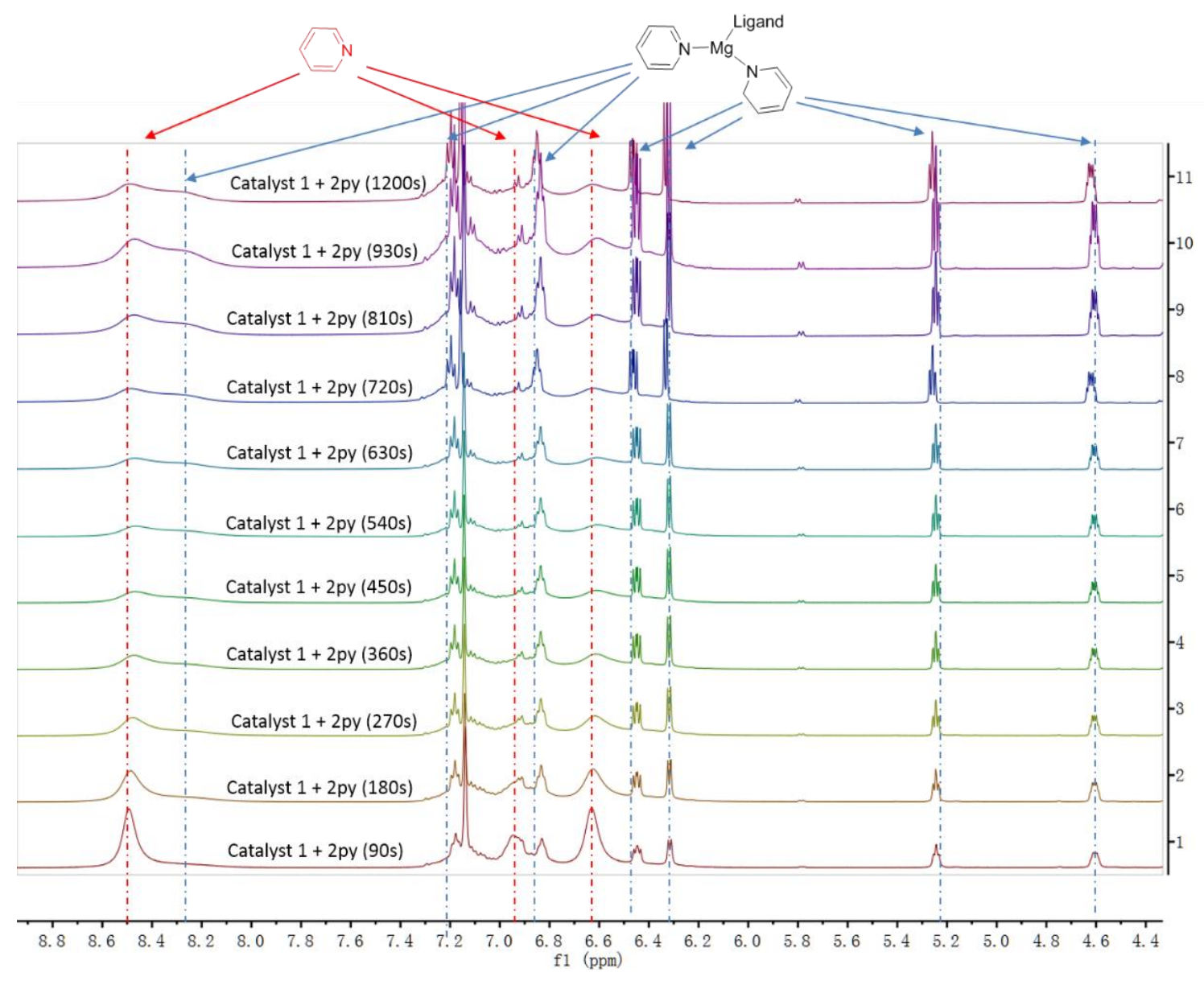

Figure S65. Stacking ${ }^{1} \mathrm{H}-\mathrm{NMR}$ spectra $\left(500 \mathrm{MHz}, \mathrm{C}_{6} \mathrm{D}_{6}\right)$ for monitoring the reaction of cat1 with 2 stoichiometric amount of pyridine. 


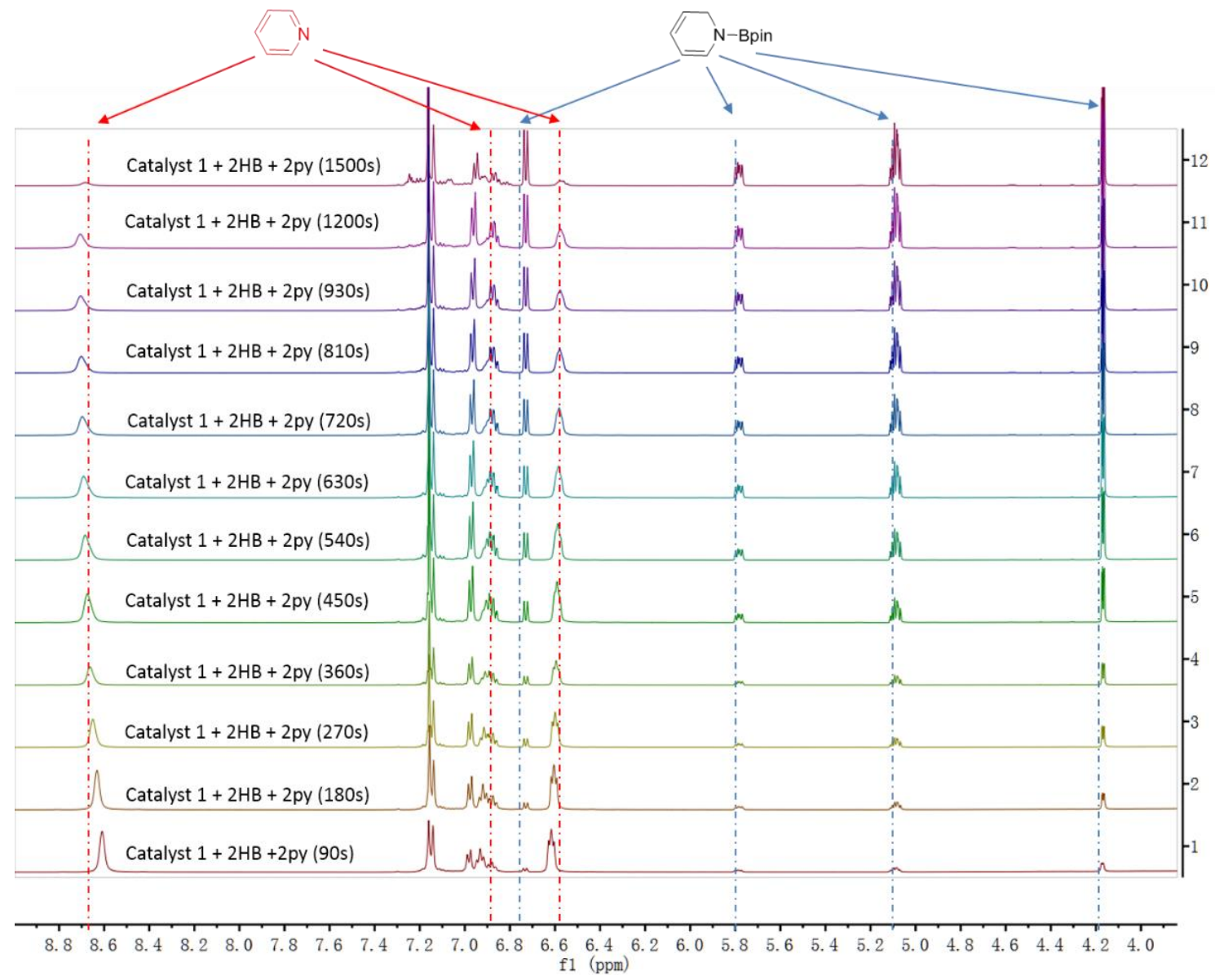

Figure S66. Stacking ${ }^{1} \mathrm{H}-\mathrm{NMR}$ spectra $\left(500 \mathrm{MHz}, \mathrm{C}_{6} \mathrm{D}_{6}\right)$ for monitoring the reaction of cat1 with 2 stoichiometric amount of pyridine and HBpin. 


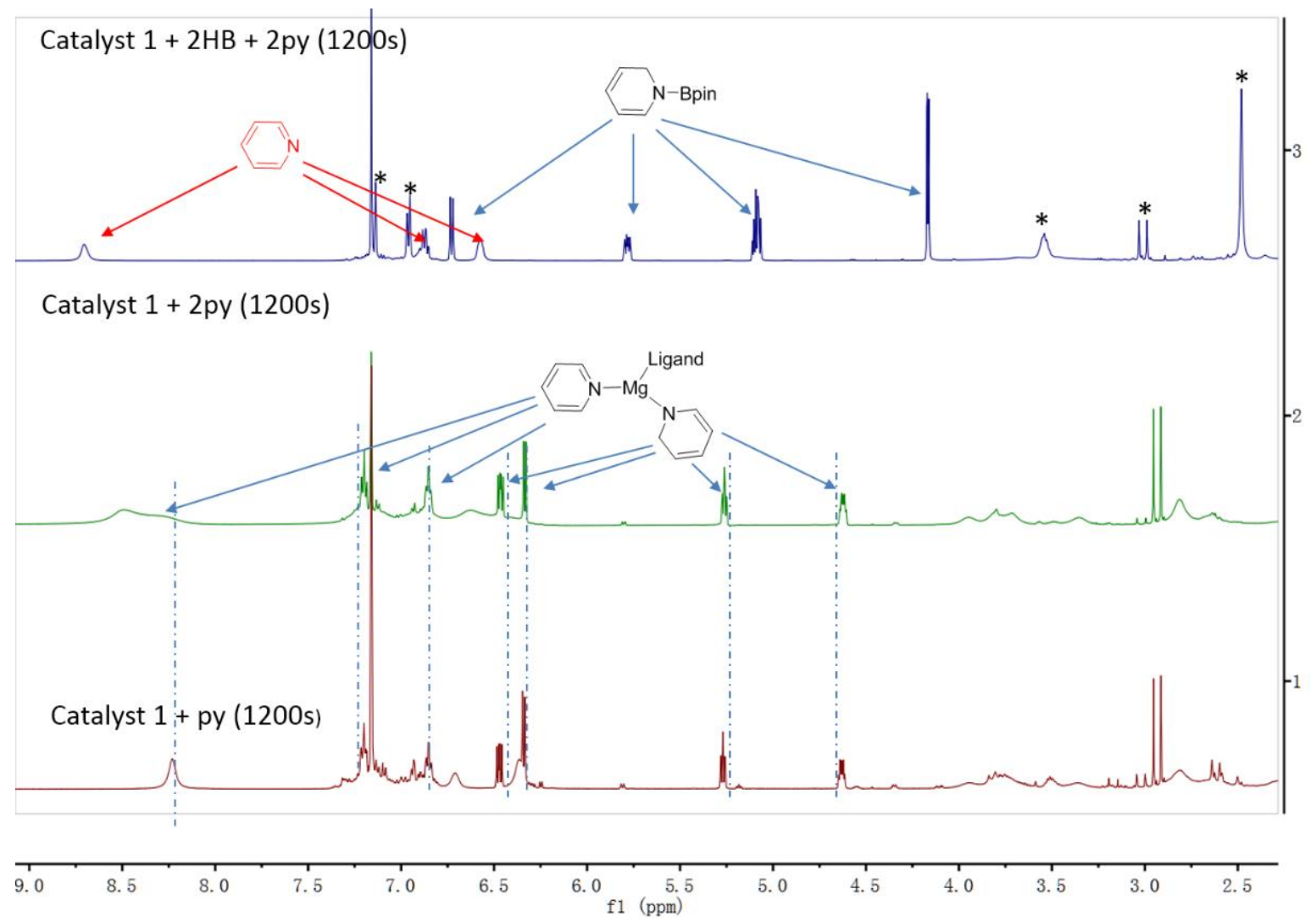

Figure S67. Summary of the ${ }^{1} \mathrm{H}-\mathrm{NMR}$ spectra $\left(500 \mathrm{MHz}, \mathrm{C}_{6} \mathrm{D}_{6}\right)$ of the reactions of cat-1+ py, cat-1+2 py and cat-1+2 py +2 HB. (* marks the signal of cat-1).

\subsection{Kinetic Analysis}

The kinetic analysis were determined by the NMR-scale reactions, in which an early time in the reaction were used to avoid the obvious change of substrate concentrations. Under these conditions, a pseudo-zero-order of the reaction can be approximated with respect to the substrate concentrations. The concentration change of products was measured depending on the integral area ratio of (1,2-dihydropyridine- $\alpha-\mathrm{H}) /\left(\mathrm{Ph}_{3} \mathrm{SiMe}\right)$. Data were fit by least-squares analysis $\left(\mathrm{R}^{2}>\right.$ 0.98) according to eq $\mathrm{S} 1$, where $\mathrm{t}$ is time and [product] is the concentration of product at time $\mathrm{t}$. The turnover frequency (TOF, $\mathrm{h}^{-1}$ ) was calculated from the least-squares determined slope (m) according to eq $\mathrm{S} 2$ where $[\mathrm{Mg}]_{0}$ is the initial concentration of $1 .^{[7]}$

$$
\begin{gathered}
\text { [product] }=m t \\
\operatorname{TOF}\left(h^{-1}\right)=\frac{60 \mathrm{~min}}{h} \times \frac{m}{[M g]_{0}}
\end{gathered}
$$




\section{Pre-catalyst 1 rate order assessment based on initial-rate kinetics}

Varying concentrations of $\mathbf{1}$ while keeping constant concentrations of HBpin and pyridine.

\begin{tabular}{|c|c|c|c|c|}
\hline$[\mathrm{Mg}]_{0}(\mathrm{M})$ & {$[\mathrm{HB}]_{0}(\mathrm{M})$} & {$[\mathrm{py}]_{0}(\mathrm{M})$} & $v_{\mathrm{i}}(\mathrm{M} / \mathrm{s})$ & $R^{2}$ \\
\hline 0.00598 & 0.377 & 0.377 & $7.099 \times 10^{-7}$ & 0.9841 \\
\hline 0.00677 & 0.377 & 0.377 & $8.516 \times 10^{-7}$ & 0.9928 \\
\hline 0.00698 & 0.377 & 0.377 & $8.873 \times 10^{-7}$ & 0.9942 \\
\hline 0.00798 & 0.377 & 0.377 & $9.808 \times 10^{-7}$ & 0.9946 \\
\hline 0.00836 & 0.377 & 0.377 & $1.006 \times 10^{-6}$ & 0.9926 \\
\hline 0.00958 & 0.377 & 0.377 & $1.224 \times 10^{-6}$ & 0.9974 \\
\hline 0.00996 & 0.377 & 0.377 & $1.716 \times 10^{-6}$ & 0.9989 \\
\hline 0.01056 & 0.377 & 0.377 & $1.983 \times 10^{-6}$ & 0.9922 \\
\hline 0.01295 & 0.377 & 0.377 & $3.208 \times 10^{-6}$ & 0.9974 \\
\hline 0.01692 & 0.377 & 0.377 & $4.283 \times 10^{-6}$ & 0.9984 \\
\hline 0.0237 & 0.377 & 0.377 & $8.501 \times 10^{-6}$ & 0.9996 \\
\hline
\end{tabular}

$[\mathrm{Mg}]_{0}$ : initial concentration of 1; $[\mathrm{HB}]_{0}$ : initial concentration of HBpin; [py $]_{0}$ : initial concentration of pyridine.

$v_{\mathrm{i}}$ : initial rate of reaction.

\section{HBpin rate order assessment based on initial-rate kinetics}

Varying concentrations of HBpin while keeping constant concentrations of $\mathbf{1}$ and pyridine.

\begin{tabular}{|c|c|c|c|c|}
\hline$[\mathrm{Mg}]_{0}(\mathrm{M})$ & {$[\mathrm{HB}]_{0}(\mathrm{M})$} & {$[\mathrm{py}]_{0}(\mathrm{M})$} & $v_{\mathrm{i}}(\mathrm{M} / \mathrm{s})$ & $R^{2}$ \\
\hline 0.00836 & 0.1378 & 0.377 & $1.933 \times 10^{-5}$ & 0.9901 \\
\hline 0.00836 & 0.1653 & 0.377 & $2.417 \times 10^{-5}$ & 0.9912 \\
\hline 0.00836 & 0.1997 & 0.377 & $3.167 \times 10^{-5}$ & 0.9872 \\
\hline 0.00836 & 0.2272 & 0.377 & $3.467 \times 10^{-5}$ & 0.9896 \\
\hline
\end{tabular}

$v_{\mathrm{i}}$ : initial rate of reaction.

\section{Pyridine rate order assessment based on initial-rate kinetics}

Varying concentrations of pyridine while keeping constant concentrations of $\mathbf{1}$ and HBpin.

\begin{tabular}{|c|c|c|c|c|}
\hline$[\mathrm{Mg}]_{0}(\mathrm{M})$ & {$[\mathrm{HB}]_{0}(\mathrm{M})$} & {$[\mathrm{py}]_{0}(\mathrm{M})$} & $v_{\mathrm{i}}(\mathrm{M} / \mathrm{s})$ & $R^{2}$ \\
\hline 0.00836 & 0.377 & 0.11 & $7.259 \times 10^{-6}$ & 0.9899 \\
\hline 0.00836 & 0.377 & 0.0825 & $3.819 \times 10^{-6}$ & 0.9815 \\
\hline 0.00836 & 0.377 & 0.09625 & $5.359 \times 10^{-6}$ & 0.9832 \\
\hline 0.00836 & 0.377 & 0.1375 & $1.0165 \times 10^{-5}$ & 0.9902 \\
\hline
\end{tabular}


$v_{\mathrm{i}}$ : initial rate of reaction.

\subsection{Activation Parameters}

Eyring plots for the reaction were plotted according to equation S3. $k$ was calculated by the lnln plot of $[\mathrm{HB}]_{0}$ vs initial rate $v_{i}$, which [pyridine $]_{0}$ and $[\mathrm{Mg}]_{0}$ were assumed didn't change in the initial reaction $(\mathrm{S} 4)$.

$$
\begin{aligned}
& \operatorname{Ln} \frac{k}{T}=\frac{\Delta \mathrm{H}^{\#}}{R} \times \frac{1}{T}+\ln \frac{k_{B}}{h}+\frac{\Delta \mathrm{S}^{\#}}{R} \\
& \frac{\mathrm{d}[P]}{\mathrm{d} t}=k_{o b s}[\mathrm{Mg}]^{1}[\text { pyridine }]^{2}[\mathrm{HBpin}]
\end{aligned}
$$

\begin{tabular}{|c|c|c|c|c|c|c|c|}
\hline$T(\mathrm{~K})$ & {$[\mathrm{Mg}]_{0}(\mathrm{M})$} & {$[\mathrm{py}]_{0}(\mathrm{M})$} & {$[\mathrm{HB}]_{0}(\mathrm{M})$} & $v_{i}(\mathrm{M} / \mathrm{s})$ & $R^{2}$ & $k$ & $R^{2}$ \\
\hline \multirow[t]{4}{*}{298} & 0.00836 & 0.377 & 0.1378 & $1.933 \times 10^{-5}$ & 0.9901 & \multirow[t]{4}{*}{1.8514} & \multirow[t]{4}{*}{0.9839} \\
\hline & 0.00836 & 0.377 & 0.1653 & $2.417 \times 10^{-5}$ & 0.9912 & & \\
\hline & 0.00836 & 0.377 & 0.1997 & $3.167 \times 10^{-5}$ & 0.9872 & & \\
\hline & 0.00836 & 0.377 & 0.2272 & $3.467 \times 10^{-5}$ & 0.9896 & & \\
\hline \multirow[t]{4}{*}{313} & 0.00836 & 0.377 & 0.2065 & $2.233 \times 10^{-5}$ & 0.9854 & \multirow[t]{4}{*}{4.576} & \multirow[t]{4}{*}{0.9865} \\
\hline & 0.00836 & 0.377 & 0.2524 & $2.667 \times 10^{-5}$ & 0.9795 & & \\
\hline & 0.00836 & 0.377 & 0.2983 & $3.183 \times 10^{-5}$ & 0.9805 & & \\
\hline & 0.00836 & 0.377 & 0.333 & $3.367 \times 10^{-5}$ & 0.9946 & & \\
\hline \multirow[t]{4}{*}{323} & 0.00836 & 0.377 & 0.2065 & $3.9177 \times 10^{-5}$ & 0.9874 & \multirow[t]{4}{*}{9.831} & \multirow[t]{4}{*}{0.9907} \\
\hline & 0.00836 & 0.377 & 0.2524 & $4.9243 \times 10^{-5}$ & 0.9789 & & \\
\hline & 0.00836 & 0.377 & 0.2983 & $5.7114 \times 10^{-5}$ & 0.9812 & & \\
\hline & 0.00836 & 0.377 & 0.333 & $6.7099 \times 10^{-5}$ & 0.9865 & & \\
\hline \multirow[t]{4}{*}{333} & 0.00836 & 0.377 & 0.2065 & $1.2867 \times 10^{-4}$ & 0.9821 & \multirow[t]{4}{*}{18.927} & \multirow[t]{4}{*}{0.9930} \\
\hline & 0.00836 & 0.377 & 0.2524 & $1.425 \times 10^{-4}$ & 0.9769 & & \\
\hline & 0.00836 & 0.377 & 0.2983 & $1.5033 \times 10^{-4}$ & 0.9848 & & \\
\hline & 0.00836 & 0.377 & 0.333 & $1.585 \times 10^{-4}$ & 0.9865 & & \\
\hline
\end{tabular}

The collected data of initial rate vs temperature

$T$ : reaction temperature; $[\mathrm{Mg}]_{0}$ : initial concentration of $\mathbf{1} ;[\mathrm{HB}]_{0}$ : initial concentration of $\mathrm{HBpin}$; [py $]_{0}$ : initial concentration of pyridine.

$v_{i}$ : initial rate of reaction. 


\subsection{Determination of the Kinetic Isotope Effect}

Preparation of DBpin: In 20mL hexane solution, $12.4 \mathrm{~mL} \mathrm{BD}$ /THF solution $(1 \mathrm{M})$ was dropwise added into equimolar 2,6-dimethylpyridine, and white precipitate appeared after two days. The precipitate then filtered and washed with hexane to get clean $\mathrm{BD}_{3} \cdot 2,6$-dimethylpyridine solid. $\mathrm{BD}_{3} \cdot 2$,6-dimethylpyridine was mixed with equimolar pinacol in $5 \mathrm{~mL} \mathrm{C}_{6} \mathrm{D}_{6}$ solution, then slowly added $\mathrm{B}\left(\mathrm{C}_{6} \mathrm{~F}_{5}\right)_{3}$ catalyst till no hydrogen gas emitted. The pure DBpin mixed with $\mathrm{C}_{6} \mathrm{D}_{6}$ solution was obtained after vacuum distillation of the reaction solution above. Its concentration was calibrated as $\mathrm{M}=1.68 \mathrm{~mol} / \mathrm{L}$ (colorless liquid, $3.9 \mathrm{~mL}$, yield: $52.8 \%$ ) by reference material of $\mathrm{Ph}_{3} \mathrm{SiMe}$. Further analysis of kinetic isotope effect has been accomplished by comparing the different initial reaction rate of 1/HBpin/py system and 1/DBpin/py system with identical conditions.

\section{Synthetic Route for DBpin within $\mathrm{C}_{6} \mathrm{D}_{6}$ Solution:}

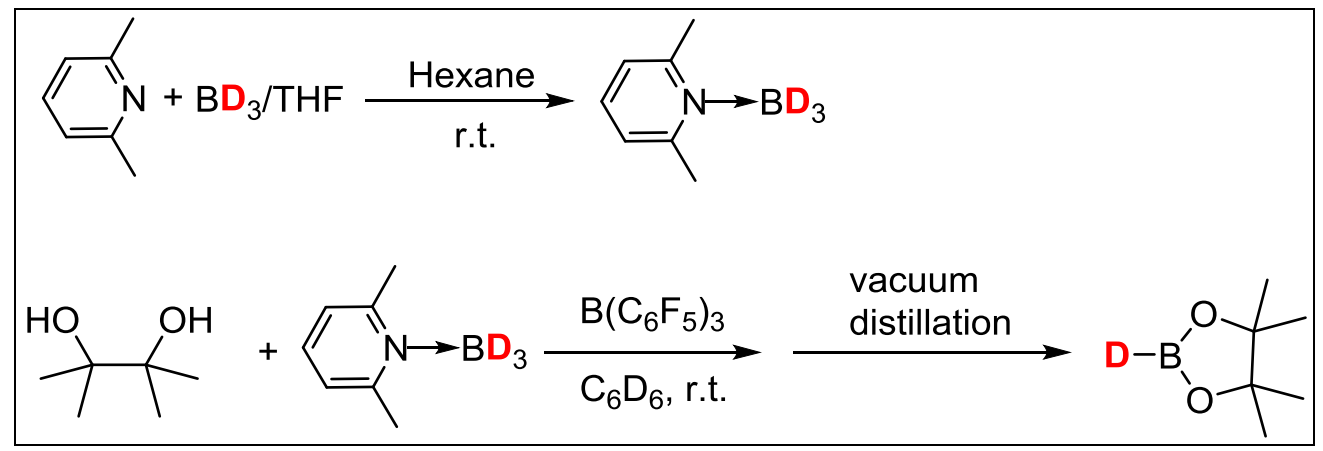

${ }^{1} \mathrm{H}$ NMR of DBpin (500 MHz, $\left.\mathrm{C}_{6} \mathrm{D}_{6}\right): \delta 0.99$ (s, 12H).

${ }^{11} \mathrm{~B}$ NMR of DBpin (160 MHz, $\left.\mathrm{C}_{6} \mathrm{D}_{6}\right): \delta 28.49$ (s). 


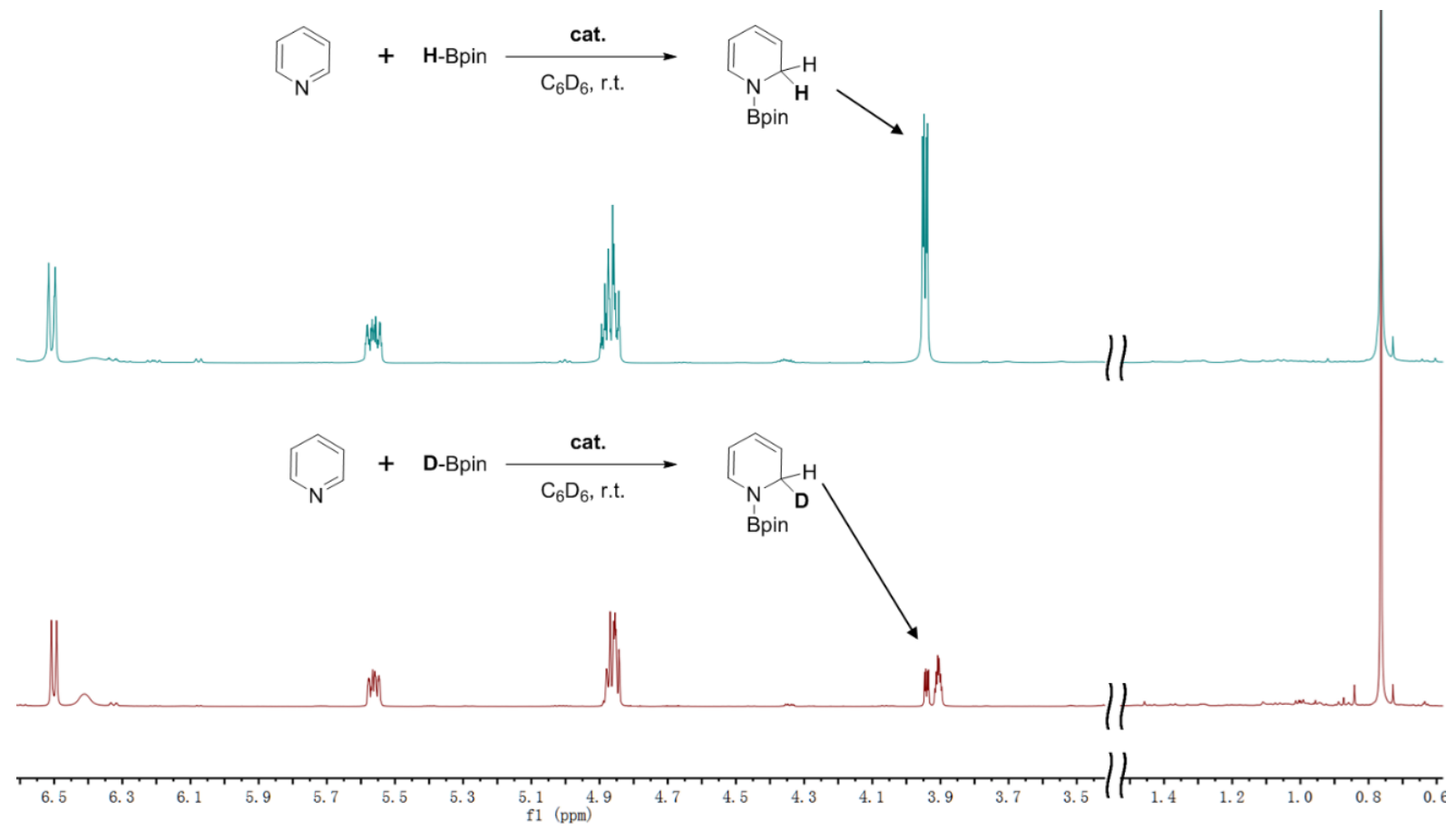

Figure S68. Compared ${ }^{1} \mathrm{H}$ NMR spectra $\left(500 \mathrm{MHz}, \mathrm{C}_{6} \mathrm{D}_{6}\right)$ of $\mathrm{HB}$ and DB reduced products.

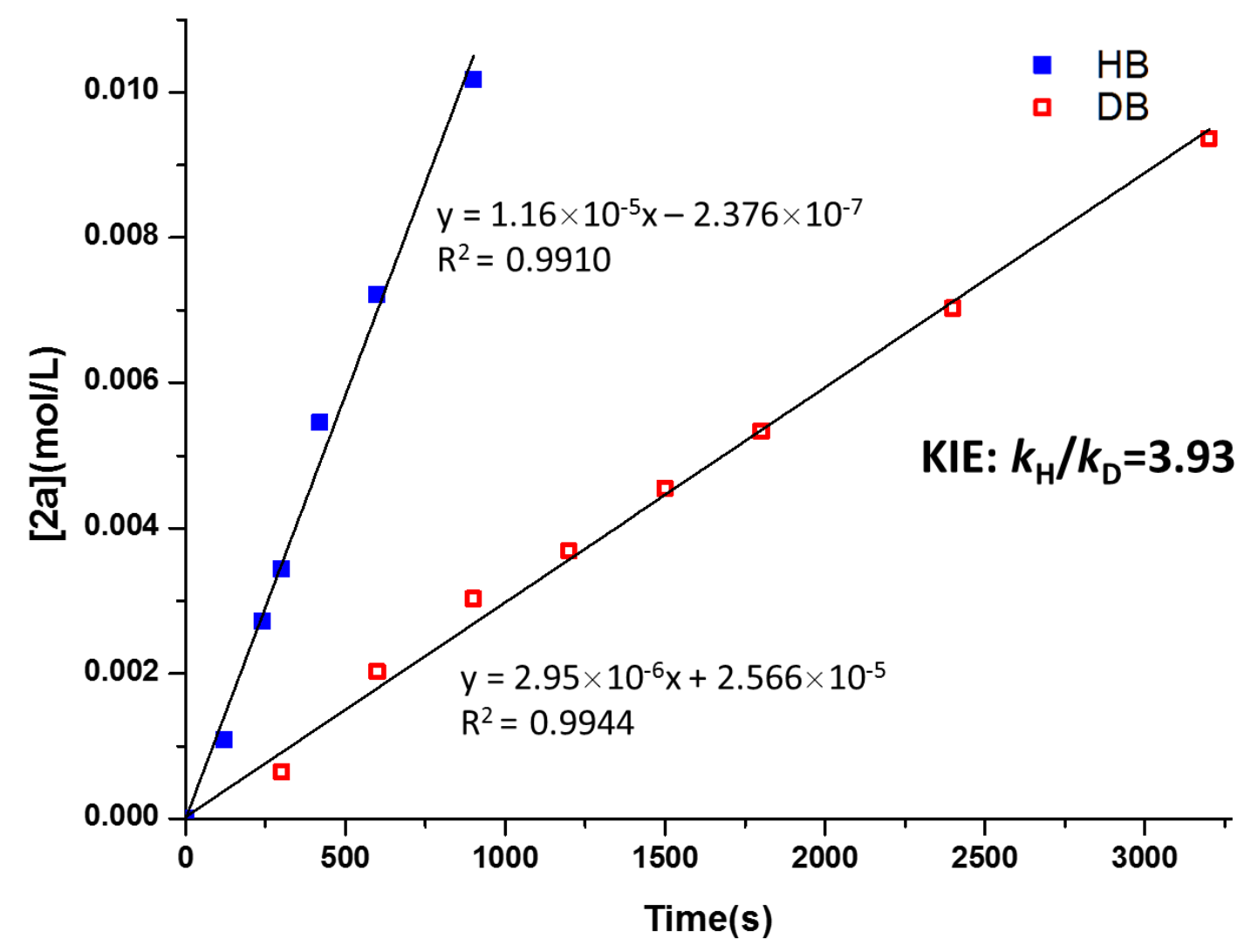

Figure S69. Initial rates of reduction of pyridine by HBpin and DBpin catalyzed by complex 1. 
Table S3. Collected data for different reaction time vs conversion

\begin{tabular}{|c|c|c|c|c|}
\hline \multicolumn{2}{|c|}{$[\mathbf{1}]:[\mathrm{DB} p i n]:[$ pyridine $]=1: 21: 21$} & \multicolumn{3}{|c|}{$[\mathbf{1}]:[\mathrm{HBpin}]:[$ pyridine $]=1: 21: 21$} \\
\hline Time $(\mathrm{s})$ & Conv. $\%$ & Time $(\mathrm{s})$ & Conv. $\%$ \\
\hline 300 & 4.7 & 120 & 6.6 \\
\hline 600 & 5.52 & 240 & 7.57 \\
\hline 900 & 6.12 & 300 & 8.01 \\
\hline 1200 & 6.51 & 420 & 9.2 \\
\hline 1500 & 7.02 & 600 & 10.25 \\
\hline 1800 & 7.49 & 900 & 12.01 \\
\hline 2400 & 8.5 & & \\
\hline 3200 & 9.89 & & \\
\hline
\end{tabular}




\section{COMPUTATIONAL DETAILS}

Molecular geometries of all complexes are optimized at the M06-2X level of density functional theory. The 6-31g (d,p) basis set are used for all atoms like $\mathrm{Mg}, \mathrm{B}, \mathrm{P}, \mathrm{C}, \mathrm{O}, \mathrm{N}$, and H. Frequency calculations at the same level of theory are also performed to identify all the stationary points as minima (zero imaginary frequencies) or transition states (one imaginary frequency) and to provide the thermal correction to free energies at $298.15 \mathrm{~K}$ and $1 \mathrm{~atm}$. The intrinsic reaction coordinate (IRC) analysis ${ }^{[8,9]}$ was carried out to confirm that all stationary points are smoothly connected to each other. To consider the solvent effect, the single-point energy calculations were performed for all the species at the M06-2X level with a same basis set 6-31G** for all atoms, on the conductorlike polarizable continuum model $(\mathrm{PCM}) \cdot{ }^{\left[{ }^{[1-12]}\right.}$ benzene was used as solvent, same as that used in experiments. All the DFT calculations were performed with GAUSSIAN 09 packages. ${ }^{[13]}$

The Gibbs free energy for each species on the potential energy profiles is taken as the sum of the thermal correction to free energies in gas-phase and the single-point energy in solution. Moreover, the free-energy correction of -4.3 or $4.3 \mathrm{kcal} / \mathrm{mol}$, proposed by Martin, Hay, and Pratt $(\mathrm{MHP})^{[14-17]}$ to correct the overestimation of entropic contributions for a reaction at $298.15 \mathrm{~K}$ and 1 atm, was added to correct the Gibbs free energies for $2: 1$ or $1: 2$ transformations. ${ }^{[18-21]}$

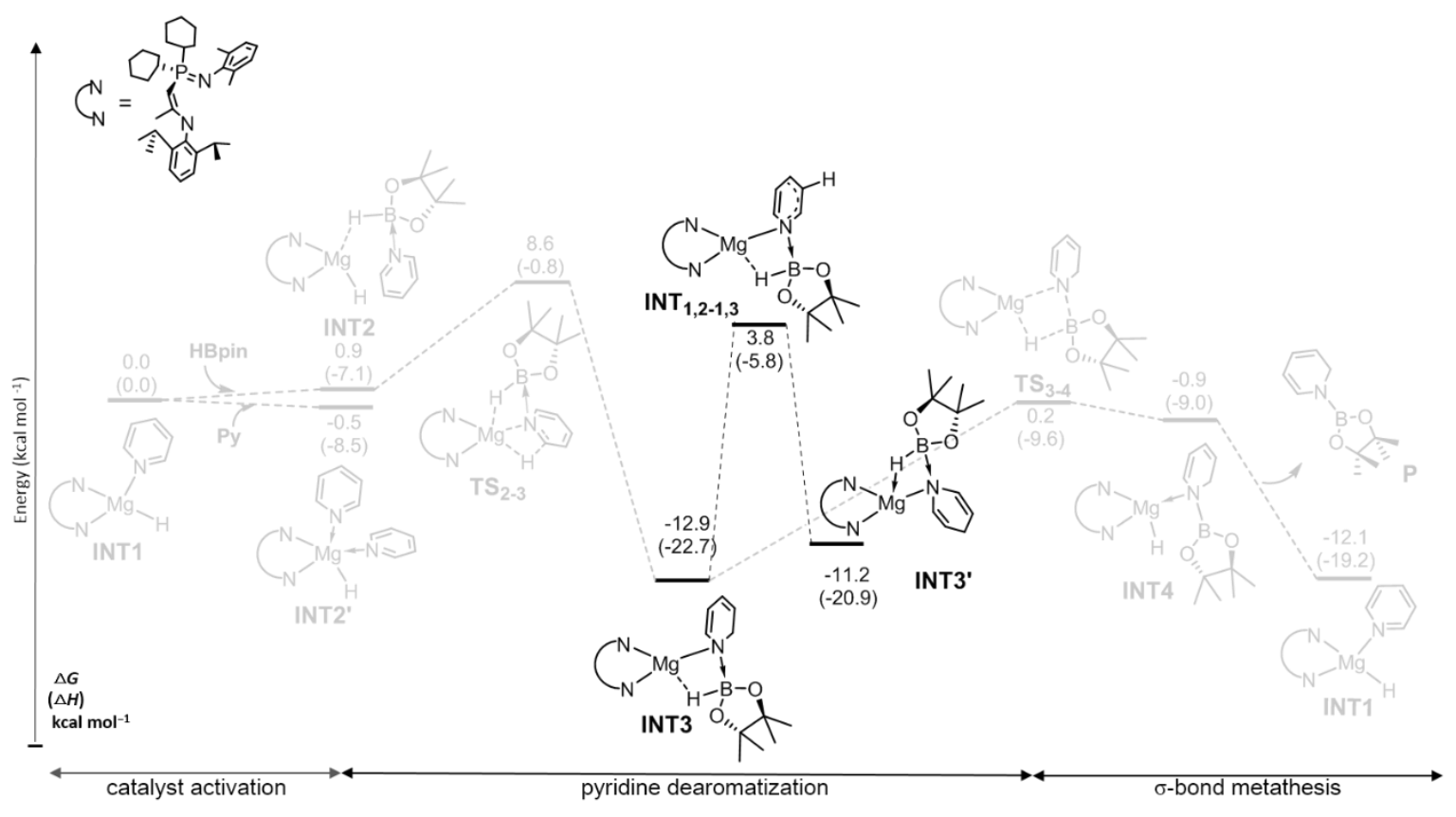

Scheme S1. DFT-derived energy profiles of alternative pathway through "1,3-DHP”state (dark line). 
DFT calculation further revealed that HBpin coordinates to the active metal center in $\mathrm{Mg}-\mathrm{H}-\mathrm{B}$ mode rather than $\mathrm{Mg}-\mathrm{O}-\mathrm{B}$ mode (as shown in Scheme S4 and Scheme S5). Indeed, the formation of the 1,2-addition product INT3o-B through TS2 $\mathbf{O}-\mathbf{B}$ needs smaller energy than INT3 (TS2 O-B $\Delta G=5.3$ vs $\mathbf{T S}_{2-3} \Delta G=7.7 \mathrm{kcal} / \mathrm{mol}$ ). However, at the rate-determination step, the $\mathrm{B}-\mathrm{H}$ bond cleavage becomes much difficult $(\Delta G=21.3 \mathrm{kcal} / \mathrm{mol})$ as compared to the $\mathrm{Mg}-\mathrm{H}-\mathrm{B}$ mode based pathway, owing to the large deformation of TS3o-B.

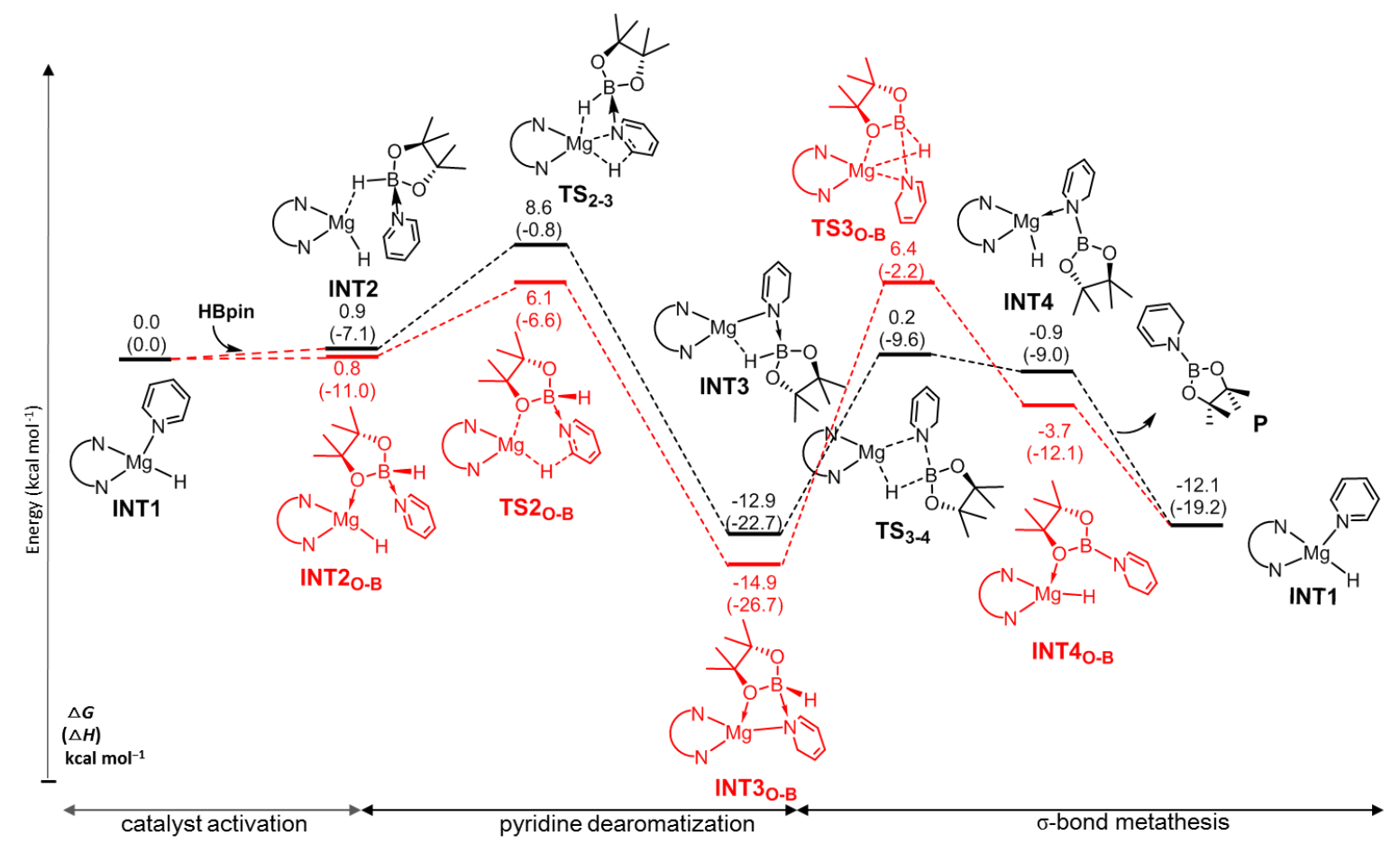

Scheme S2. DFT-derived energy profiles of alternative "Mg-O-B bridge" based 1,2-addition pathway (red line) vs "Mg-H-B bridge" based 1,2-addition pathway (dark line). 


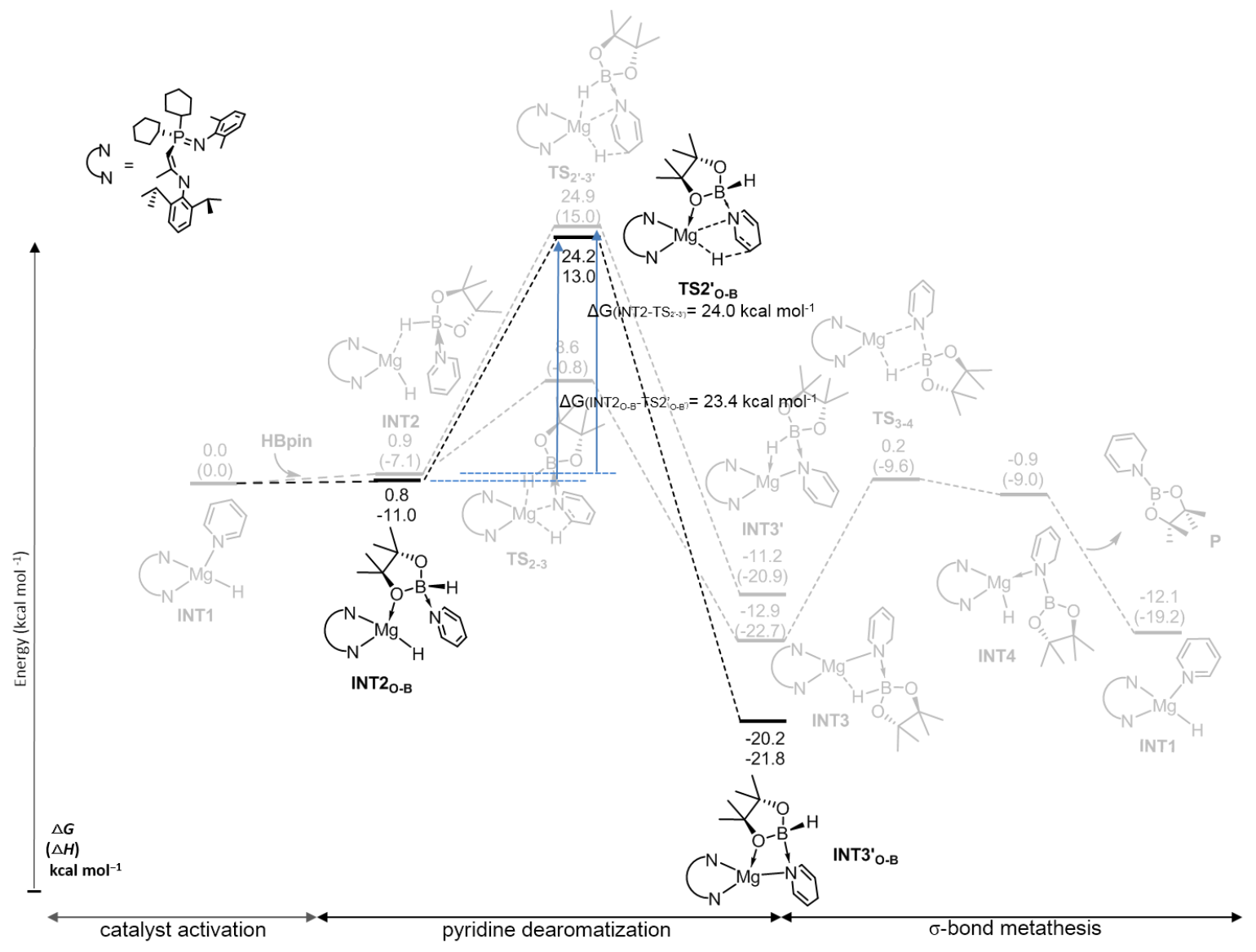

Scheme S3. DFT-derived energy profiles of alternative "Mg-O-B bridge" based 1,4-addition pathway (dark line).

Table S4. Calculated thermodynamic corrections, solution-phase single-point energies in benzene and solution-phase Gibbs free energies in toluene of all the intermediates and transition states (in Hartree).

\begin{tabular}{|c|c|c|c|}
\hline & $\begin{array}{c}\text { Gas-Phase Gibbs } \\
\text { Free Energies } \\
\text { Corrections }\end{array}$ & $\begin{array}{c}\text { Solution-Phase } \\
\text { Single-Point } \\
\text { Energies }\end{array}$ & $\begin{array}{c}\text { Solution-Phase Gibbs } \\
\text { Free Energies }\end{array}$ \\
\hline pre-cat & 1.519861 & -4032.01926 & -4030.499399 \\
\hline
\end{tabular}




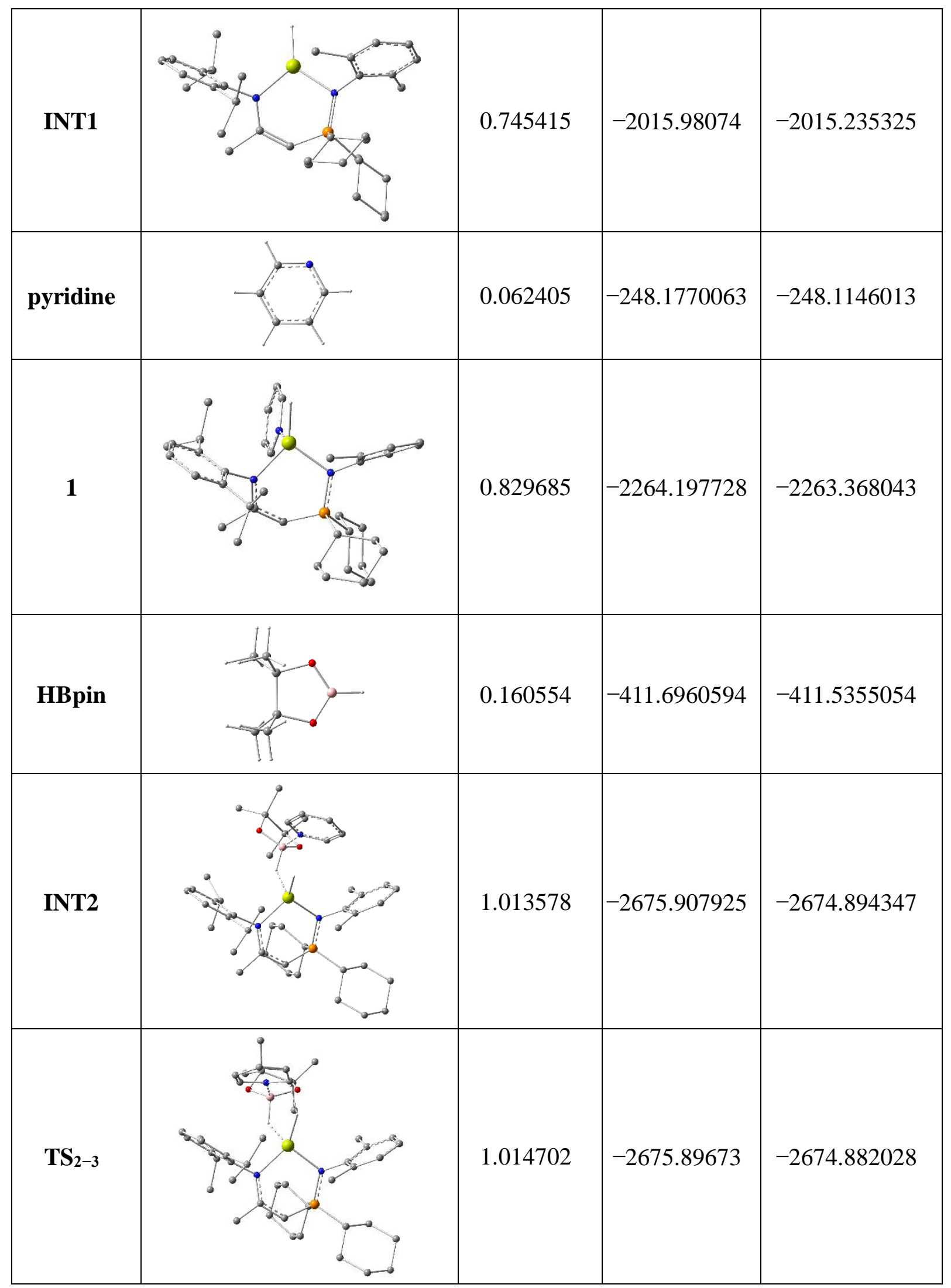




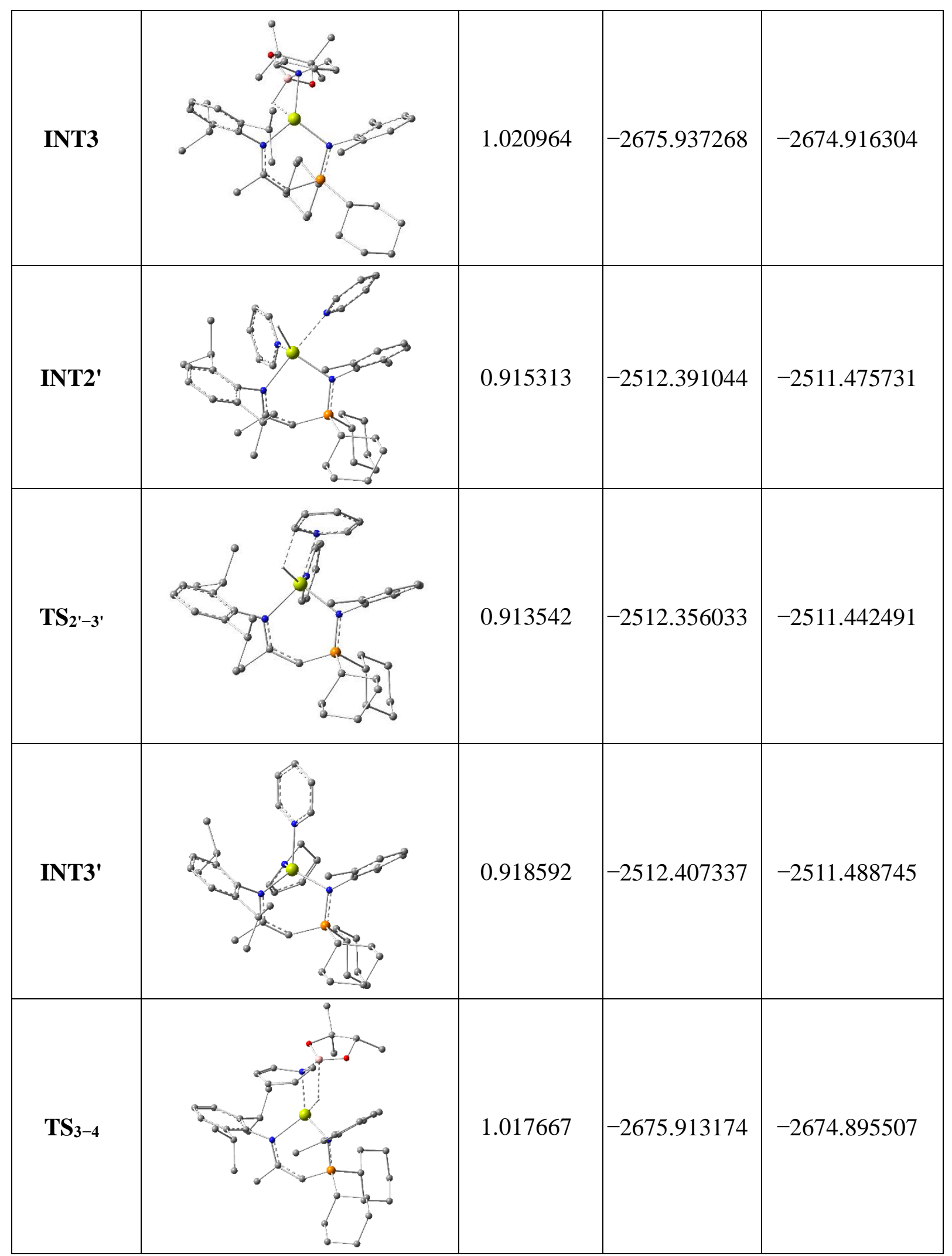




\begin{tabular}{|l|l|l|l|l|}
\hline INT4 & & & & \\
\hline
\end{tabular}

\section{REFERENCES}

[1] H. Xie, X. Liu, D. Cui, Organometallics 2017, 36, 3597-3604.

[2] S. Gong, H. Ma, Dalton Trans. 2008, 3345-3357.

[3] V. L. Weidner, C. J. Barger, M. Delferro, T. L. Lohr, T. J. Marks, Acs Catal. 2017, 7, 1244-1247.

[4] R. Rochat, M. J. Lopez, H. Tsurugi, K. Mashima, ChemCatChem 2016, 8, 10-20.

[5] D. Mukherjee, A. Ellern, A. D. Sadow, Chem. Sci. 2014, 5, 959-964.

[6] H. Xie, X. Hua, B. Liu, C. Wu, D. Cui, J. Organomet. Chem. 2015, 798, 335-340.

[7] A. S. Dudnik, V. L. Weidner, A. Motta, M. Delferro, T. J. Marks, Nat. Chem. 2014, 6, 1100-1107.

[8] K. Fukui, J. Phys. Chem. 1970, 74, 4161-4163.

[9] K. Fukui, Acc. Chem. Res. 1981, 14, 363-368.

[10] E. Cances, B. Mennucci, J. Tomasi, J. Chem. Phys. 1997, 107, 3032-3041.

[11] B. Mennucci, J. Tomasi, J. Chem. Phys. 1997, 106, 5151-5158.

[12] G. Scalmani, M. J. Frisch, J. Chem. Phys. 2010, 132, 114110.

[13] H. Jiang, W. Fu, H. Chen, Chem. Eur. J. 2012, 18, 11884-11888.

[14] A. Bennaim, Y. Marcus, J. Chem. Phys. 1984, 81, 2016-2027.

[15] M. D. Tissandier, K. A. Cowen, W. Y. Feng, E. Gundlach, M. H. Cohen, A. D. Earhart, J. V. Coe, T. R. Tuttle, J. Phys. Chem. A 1998, 102, 7787-7794.

[16] C. P. Kelly, C. J. Cramer, D. G. Truhlar, J. Phys. Chem. B 2006, 110, 16066-16081.

[17] P. E. M. Lopes, B. Roux, A. D. MacKerell, Jr., Theor. Chem. Acc. 2009, 124, 11-28.

[18] C. Song, S. Qu, Y. Tao, Y. Dang, Z.-X. Wang, Acs Catal. 2014, 4, 2854-2865.

[19] S. Qu, H. Dai, Y. Dang, C. Song, Z.-X. Wang, H. Guan, Acs Catal. 2014, 4, 43774388.

[20] S. Qu, Y. Dang, C. Song, J. Guo, Z.-X. Wang, Acs Catal. 2015, 5, 6386-6396.

[21] M. Wen, F. Huang, G. Lu, Z.-X. Wang, Inorg. Chem. 2013, 52, 12098-12107. 